\title{
CONSPECTVS AVCTORVM OPERVMQVE LAVDATORVM
}

In hoc conspectu enumeravi libros monographicos et collectiones auctorum variorum nec non editiones titulorum iam ad finem redactas. In notis bibliographicis tam ad titulos plene editos adscriptis quam in addendis et corrigendis inscriptionum iam supra editarum mentionem collectionum lexicorumque prosopographicorum operumque aliorum nonnullorum, quae libris studiisque recentioribus publici iuris factis hodie usui tantum minori essent aut res notas solum repeterent, generaliter omisi. Quae opera quidem in hac bibliographia comprehenduntur; ad titulos singulos laudantur imprimis, si res notabiles aliunde omissas vel minus recte propositas continent, praeterea, si titulus, qui tractatur, bibliographia alia caret, item, si opus bibliographiam continet ampliorem ea, quae hoc Corpus praebet.

Аввате, Guida I-II = E. Аввате, Guida dell'Abruzzo I-II, Roma 1903.

Abbatia S. Clementis = G. SARtorelli - F. Parenti L. Mancini - L. Iannucci, Abbatia S. Clementis, Pescara 1975.

Aвramenko, Munizipale Mittelschicht $=$ A. Aвramenko, Die munizipale Mittelschicht im kaiserzeitlichen Italien: zu einem neuen Verständnis von Sevirat und Augustalität (Europäische Hochschulschriften 547), Frankfurt a. M. Berlin - Bern - New York - Paris - Wien 1993.

Aвsı, Préfets $=$ M. AвsıL, Les préfets du prétoire d'Auguste à Commode. 2 avant Jésus-Christ - 192 après Jésus-Christ, Paris 1997.

Abruzzo nel Medioevo = E. Tiвoni (Ed.), Abruzzo nel Medioevo, Pescara 2003

Accivile - Gualtieri - Nannarone, Scanno = R. Accivile - M. Gualtieri - R. Nannarone, Scanno. Guida storica artistica (da un manoscritto inedito di Mons. G. CELIDONIO), Roma 1974

Acque, grotte e Dei = M. Pacciarelli (Ed.), Acque, grotte e Dei. 3000 anni di culti preromani in Romagna, Marche e Abruzzo, Imola (BO) 1997.

Acta Barcelona = M. Mayer y Olivé - G. Baratta A. GuZmán Almagro (Ed.), XII Congressus Internationalis Epigraphiae Graecae et Latinae. Provinciae Imperii Romani inscriptionibus descriptae. Barcelona, 3-8 Septembris 2002. Acta I-II (Monografies de la Secció HistòricoArqueològica 10), Barcelona 2007.

Acta Cambridge $=$ Acta of the Fifth International Congress of Greek and Latin Epigraphy Cambridge 1967, Oxford 1971.

Acta Colloquii Epigraphici Latini $=$ H. Solin - O. SAlOMIES U.-M. LierTz (Ed.), Acta Colloquii Epigraphici Latini Helsingiae 3.-6. Sept. 1991 habiti (Soc. Scient. Fennica, Comment. Human. Litt. 104), Helsinki 1995.

Actes Constantza $=$ Actes du VII ${ }^{e}$ Congrès international d'Épigraphie grecque et latine. Constantza, 9-15 septembre 1977, București - Paris 1979.

Actes $d u$ Colloque Degrassi = Epigrafia . Actes $d u$ Colloque international d'epigraphie latine en mémoire de Attilio Degrassi pour le centenaire de sa naissance, Rome, 27-28 mai 1988 (Coll. de l'École Fr. de Rome 143), Paris - Rome 1991.

Actes Nimes = M. Christol - O. Masson (Ed.), Actes $d u X^{e}$ Congrès international d'Épigraphie grecque et latine. Nîmes, 4-9 octobre 1992 (Histoire ancienne et médiévale 42), Paris 1997

Ad itum liberum = O. Merisalo - R. Vainio (Ed.), Ad itum liberum. Essays in honour of Anne Helttula, Jyväskylä 2007.

Affreschi $=$ L. ANGELETTI (Ed.), Gli affreschi della chiesa di S. Scolastica a Norcia, Norcia (PG) 1981.
Ager Campanus = G. Franciosi (Ed.), Ager Campanus. Atti del Convegno Internazionale "La storia dell'Ager Campanus, $i$ problemi della limitatio e sua lettura attuale». Real sito di S. Leucio, 8-9 giugno 2001, Napoli 2002.

Agostinoni, Il Fucino = E. Agostinoni, Il Fucino, Bergamo 1908.

Aia dei musei = F. De Sanctis - R. Del Monaco - A. SaraGOSA - D. Villa (Ed.), L'aia dei musei, Avezzano (AQ) 2012.

AISCOM XVIII = Atti del XVIII Colloquio dell'Associazione Italiana per lo Studio e la Conservazione del Mosaico con il patrocinio del Ministero per i Beni e le Attività Culturali, Cremona, 14-17 marzo 2012, Tivoli (RM) 2013.

Akte Wien = Akte des IV. Internationalen Kongresses für griechische und lateinische Epigraphik. Wien, 17. bis 22. September 1962, Wien 1964.

Akten München = Akten des VI. Internationalen Kongresses für griechische und lateinische Epigraphik. München 1972 (Vestigia 17), München 1973.

Alba Fucens I-II = J. Mertens (Ed.), Alba Fucens. Rapports et études I-II (Inst. Hist. Belge de Rome. Études 12-13), Bruxelles - Rome 1969.

Albino, Ricordi = P. Albino, Ricordi storici e monumentali del Sannio Pentro e della Frentania I, Campobasso 1879.

Alessio - De Giovanni, Preistoria e protostoria = G. Alessio - M. DE Giovanni, Preistoria e protostoria linguistica dell'Abruzzo, Lanciano - Chieti 1983.

AlFÖLDY, Bevölkerung = G. AlFÖLDY, Bevölkerung und Gesellschaft der römischen Provinz Dalmatien. Mit einem Beitrag von A. Mócsy, Budapest 1965.

ALFÖLDY, Epigrafia augustea = G. ALFÖLDY, Studi sull'epigrafia angustea e tiberiana di Roma (Vetera 8), Roma 1992.

Alföldy, Estudios = D. Gorostidi Pi (Ed.), Géza Alföldy. Estudios Tarraconenses, Tarragona 2017.

Alföldy, Fasti Hispanienses = G. AlföldDY, Fasti Hispanienses. Senatorische Reichsbeamte und Offiziere in den spanischen Provinzen des römischen Reiches von Augustus bis Diokletian, Wiesbaden 1969.

Alföldy, Gallia Cisalpina = G. Alföldy, Städte, Eliten und Gesellschaft in der Gallia Cisalpina: Epigraphische Untersuchungen (HABES 30), Stuttgart 1999.

AlFÖLDY, Heeresgeschichte = G. AlFöLDY, Römische Heeres geschichte. Beiträge 1962-1985 (Mavors 3), Amsterdam 1987.

Alföldy, Hilfstruppen = G. Alföldy, Die Hilfstruppen der römischen Provinz Germania inferior (Epigr. Studien 6), Düsseldorf 1968.

AlföLdy, Konsulat = G. AlFöLdy, Konsulat und Senatorenstand unter den Antoninen. Prosopographische Untersuchungen zur senatorischen Führungsschicht (Antiquitas 1. 27), Bonn 1977. 
ALFöLDY, Legionslegaten = A. ALFÖLDY, Legionslegaten der römischen Rheinarmeen (Epigr. Studien 3), Graz - Köln 1967.

Alföldy, Noricum = G. AlfÖldy, Noricum, London Boston 1974

Alföldy, Personennamen = G. Alföldy, Die Personennamen in der römischen Provinz Dalmatia (Beiträge zur Namenforschung, N. F., Beibeft 4), Heidelberg 1969.

AlFÖLDY, Rolle = G. AlFÖLDY, Die Rolle des Einzelnen in der Gesellschaft des römischen Kaiserreiches. Erwartungen und Wertmaßstäbe (Sitzungsberichte der Heidelberger Akademie der Wissenschaften. Phil.-Hist. Klasse 8), Heidelberg 1980.

Alife. Anfiteatro = G. Soricelli - E. A. Stanco (Ed.), Alife. L'Anfiteatro Romano, Piedimonte Matese (CE) 2009.

Alife romana = Alife romana: contributo alla conoscenza delle opere publiche e private, Napoli 1982.

Allega, Gagliano Aterno = D. Allega, Gagliano Aterno. La storia - I monumenti - Il folklore, Pratola Peligna (AQ) 1977.

Almar, Inscriptiones = K. P. Almar, Inscriptiones Latinae. Eine illustrierte Einführung in die lateinische Epigraphik (Odense University Classical Studies 14), Odense 1990.

Altmann, Grabaltäre $=$ W. Altmann, Die römischen Grabaltäre der Kaiserzeit, Berlin 1905.

Álvarez Melfro, Matronae equestres = A. Álvarez Melero, Matronae equestres. La parenté féminine des chevaliers romains originaires des provinces occidentales sous le Haut-Empire romain (I ${ }^{e r}-I I I^{e}$ siècles) (Inst. Belge de Rome. Études 4), Bruxelles - Rome 2018.

Alvino, Forum Novum = G. Alvino, La valle del Tevere Forum Novum e il suo territorio, Roma 1999.

Alvino, Sabina = G. Alvino, La Sabina, Roma 1999.

Alvino, Trebula Mutuesca = G. Alvino, Trebula Mutuesca . La riscoperta di un antico centro sabino, Roma 1995.

Aмatı, $m s .=$ G. Amatı, Libri saec. XIX ineunte manu scripti in Bibliotheca Apostolica Vaticana adservati sign. Vaticani Latini 9734-9782 (etiam vide infra Buonocore, Codices Vaticani).

Amelotti, Il testamento romano $=\mathrm{M}$. Amelotti, Il testamento romano attraverso la prassi documentale. I. Le forme classiche di testamento, Milano 1966.

Amicitia = K. MustaKallio - Chr. KrötZl (Ed.), De amicitia. Friendship and Social Networks in Antiquity and the Middle Ages (Acta Inst. Rom. Finl. 36), Roma 2009.

Amiconi, Appunti = F. M. Amiconi, Appunti di archeologia cerchiese, Roma [1987]

Amplero = C. LetTA - M. Paoletti (Ed.), Amplero. Archeologia e storia di un centro italico-romano. 20 anni di ricerche. Catalogo della mostra. Collelongo (AQ) - Palazzo Botticelli, 16 agosto-31 dicembre 1989, Sambuceto (CH) 1989.

Analecta Brixiana I = A. VAlvo - G. MANzoni (Ed.), Analecta Brixiana I. Contributi dell'Istituto di Filologia e Storia dell'Università Cattolica del Sacro Cuore, Milano 2004.

Analecta Brixiana II = A. VAlvo - R. GAZICH (Ed.), Analecta Brixiana II. Contributi dell'Istituto di Filologia e Storia dell'Università Cattolica del Sacro Cuore, Milano 2007.

Anamali - Ceka - Deniaux, Inscriptions latines d'Albanie = S. Anamali - H. Ceka - É. Deniaux, Corpus des inscriptions latines d'Albanie (Coll. de l'École Fr. de Rome 410), Rome 2009.

Ancient Documents = J. Bodel - N. Dimitrova (Ed.), Ancient Documents and their Contexts. First North American Congress of Greek and Latin Epigraphy (2011) (Brill Studies Epigraphy 5), Leiden 2011.

Ancient History = K. Ascani - V. Gabrielsen - K. Kvist A. H. Rasmussen (Ed.), Ancient History Matters. Studies Presented to Jens Erik Skydsgaard on His Seventieth Birth- day (Anal. Rom. Inst. Dan. Supplementum 30), Roma 2002.

Ancient Medicine $=$ PH. J. VAN DER EIJK - H. F. J. HorstMANSHOFF - P. H. SChrijvers (Ed.), Ancient Medicine in its Socio-Cultural Context. Papers read at the Congress held at Leiden University, 1-15 April 1992, Amsterdam Atlanta (GA) 1995.

Andermahr, Totus in praediis = A. Andermahr, Totus in praediis. Senatorischer Grundbesitz in Italien in der Frühen und Hohen Kaiserzeit (Antiquitas 3. 37), Bonn 1998.

ANDREAU, La vie financière $=\mathrm{J}$. ANDREAU, La vie financière dans le monde romain. Les métiers de manieurs d'argent (IV siècle av. J.-C. - III siècle ap. J.-C.) (Bibl. des Écoles Fr. d'Athènes et de Rome 265), Roma 1987.

Andreau, Les affaires = J. AndreAu, Les affaires de Monsieur Jucundus (Coll. de l'École Fr. de Rome 19), Rome 1974.

ANDREOZZI, Antiche diocesi = A. AndreozzI, Le antiche diocesi sabine: Cures, Nomentum, Roma 1973.

ANELli, Esposizione = L. ANelli, Esposizione degli oggetti esistenti nel Gabinetto di Vasto compilata su documenti raccolti dal Betti, dal Marchesani e dall'Altea, Vasto (CH) 1899.

Anelli, Histonium ed il Vasto = L. Anelli, Histonium ed il Vasto attraverso i secoli, Vasto (CH) 1929

AnElli, Ricordi di storia vastese ${ }^{3}=\mathrm{L}$. ANELli, Ricordi di storia vastese $e^{3}$, Vasto $(\mathrm{CH}) 1906$

Anelli, Ricordo di Vasto = L. Anelli, Ricordo di Vasto, Bergamo 1909

Anfiteatro Flavio $=$ Anfiteatro Flavio. Immagine - Testimonianze-Spettacoli. Testi di M. L. ConforTO ... [et AL.; a cura di A. M. Reggiani], Roma 1988.

Angelini, S. Cesidio = E. Angelini, Il braccio di S. Cesidio, Pescara 1985.

Angelini, Trasacco nell'Impero = E. Angelini, Trasacco nell'Impero Romano, Trasacco (AQ) 1978.

Angelini, Trasacco prima di Roma = E. Angelini, Trasacco prima di Roma, Pescara 1973.

Angelini Rota, Museo = G. Angelini Rota, Il Museo civico di Spoleto, Spoleto (PG) 1928

Angelini Rota, Spoleto = G. Angelini Rota, Spoleto e il suo territorio, Spoleto (PG) 1920.

Annaei = I. Gualandri - G. Mazzoli (Ed.), Gli Annaei. Una famiglia nella storia e nella cultura di Roma imperiale. Atti del Convegno internazionale di Milano - Pavia, 2-6 maggio 2000, Como 2003.

Anonymus, ms. I = Inventari e descrizioni delle diverse raccolte conservate nel Museo (liber temporis spatio inter a. 1850-1931 comprehenso exaratus in bibliotheca communali Vastensi sine inv. n. servatus).

Anonymus, $m s . \mathrm{II}=$ Catalogo degli oggetti raccolti nel Museo Archeologico di Istonio (liber manu scriptus saec. XX in. in bibliotheca communali Vastensi sine inv. n. servatus).

Anonymus, ms. III = Dell'antica Cursuli (liber saec. XVIII ex autographo eiusdem aetatis in aedibus Paoletti vel Antonelli in vico Legogne di Norcia olim servato manu descriptus, qui liber in bibliotheca communali Nursina Fondo Carte Cesare Lalli sine inv. n. exstabat; postquam terrae motus d. 24 m. Aug. et d. 30 m. Oct. a. 2016 universum oppidum diruerunt, Spoleto est translatus).

ANonymus, ms. IV = Delli Annali di Norcia (liber a. 1600 exaratus in archivo secreto communali Nursino exstabat sine inv. n.; postquam terrae motus d. $24 \mathrm{~m}$. Aug. et d. $30 \mathrm{~m}$. Oct. a. 2016 universum oppidum diruerunt, Spoleto est translatus).

Anonymus, ms. $\mathrm{V}=$ Opusculum cui incipit Nursiam urbem antiquissimam fuisse Romanorum Praefecturam, saec. XVIII medio exaratum, invenitur inter folia CCCVIIIICCCXIV libri manu scripti Memorie di Norcia nempe Zibaldone Fusconi olim servati in bibliotheca communali 
Nursina sine inv. n.; postquam terrae motus d. 24 m. Aug. et d. 30 m. Oct. a. 2016 universum oppidum diruerunt, liber Spoleto est translatus.

Anonymus, $m s . \mathrm{VI}=$ Anonymus ordinis fratrum cocullatorum nuncupatus Minorita Norcino (attamen videtur fuisse Franciscus Antonius Rocci, orgine Nursinus, ut elicitur ex Lalli, Miscellanea n. 46), in libro, cui titulus Memorie antiche e recenti dell'antichissima città di Norcia, temporis spatio inter saec. XVIII ex. et XIX in. comprehenso manu scripto olim servato apud archivum dioecesanum Norcia sine inv. n., vol. I-II; postquam terrae motus d. 24 m. Aug. et d. 30 m. Oct. a. 2016 universum oppidum diruerunt, liber Spoleto est translatus.

Anonymus, ms. VII = Marmi Antichi quali al p(rese)nte se ritrouano dentro la Città de Alife, eccetto quattro quali sono in Piedimonte, tre in lo Monasterio di S(an)to Saluatore, et uno in S(an)to Nazaro, trascritti per inviarsi al S(igno)r Franc(esc)o Ant(oni)o de Tomasi mio S(igno)re (fasciculus f. 32-37 insertus in codice saec. XVII olim Alessii Symmachi Mazzocchi nunc in Bibliotheca Apostolica Vaticana servato sub num. Vaticanus Latinus 10593. Auctor ab Accursio pendere videtur).

ANONYMUS, ms. VIII = Iscrizioni antiche raccolte dal Mazzocchi ed altre esistenti ne' quartieri Stella, S. Carlo all'Arena, S. Lorenzo ed altri luoghi di Napoli e Pozzuoli (fasciculus chartarum plerisque manibus saec. XVIII-XIX exaratarum, quas Constantino CoRvisieri [1822-1898] possedit, nunc servatus Romae apud Archivio di Stato sign. Miscellanea Corvisieri, b. 6, fasc. 190 [vide E. Casanova, Gli Archivi Italiani 7, 1920, 20-48]; in f. 89r-92r transcripti sunt tituli viginti sex Telesini, scil.: Inscriptiones Thelesinae 〈add. in Campania〉). Gratias ago Umberto Soldovieri, qui me comiter certiorem fecit.

Anonymus, $m s$. IX = Libellus redactus a. 1876 hic et illic in marginibus saepe excisis madore maculatus signatus $b .406$ fasc. 46 sine autem foliorum nota, nunc servatus Capuae in Biblioteca del Museo Campano, titulos Allifanos quosdam complectens.

Antiche genti d'Italia = P. G. Guzzo - S. Moscati - G. SusinI (Ed.), Antiche genti d'Italia, Roma 1994.

Antichità paleocristiane = L. GiUlia - A. QuacQuarelli (Ed.), Antichità paleocristiane e altomedievali del Sorano. Atti del Convegno di Studi, Sora, 1-2 dicembre 1984, Sora (FR) 1985.

Antike Lebenswelten = P. MAuritsCH (Ed.), Antike Lebenswelten: Konstanz - Wandel - Wirkungsmacht. Festschrift für Ingomar Weiler zum 70. Geburtstag (Philippika 25), Wiesbaden 2008.

Antinori, ms. = A. L. ANTINORI, Libri saec. XVIII manu scripti signati n. XLIII-XLVI in bibliotheca publica Aquilana Salvatore Tommasi servati, qui syllogen inscriptionum in Samnio repertarum continent.

Antinoriana I-IV = Antinoriana. Studi per il bicentenario della morte di Antonio Ludovico Antinori I-IV, L'Aquila 1978/ 79.

Antinum = Antinum e la Valle Roveto nell'antichità. Atti del I Convegno di archeologia, Civita d'Antino, 16 settembre 1990, Civita d'Antino (AQ) 1992.

Antiqua Beneventana $=$ P. CARUso (Ed.), Antiqua Beneven tana. La storia della città romana attraverso la documentazione epigrafica (Supplemento a La Provincia Sannitica n. s. 33, 1, 2013), Benevento 2013.

Antiquarium Celio = G. L. Gregori (Ed.), La collezione epigrafica dell'Antiquarium Comunale del Celio. Inventario generale. Inediti - Revisioni - Contributi al riordino (Tituli 8), Roma 2001.

Antolini, Iscrizioni $=\mathrm{S}$. Antolini, Le iscrizioni latine rupestri della regio IV Augustea. Edizione, commento e inqua- dramento storico-archeologico (DASP. Documenti per la storia d'Abruzzo 17), L'Aquila 2004.

Anton Ludovico Antinori = Anton Ludovico Antinori e il $\mathrm{II}^{\circ}$ centenario della sua nascita. Pubblicazione della Società di Storia patria negli Abruzzi, Aquila 1904

Antonio Agustin = M. H. CraWford (Ed.), Antonio Agustin between Renaissance and Counter-Reform (Warburg Institut Surveys and Texts 24), London 1993.

Antropologia e archeologia = V. Nizzo - L. LA Rocca (Ed.), Antropologia e archeologia a confronto: rappresentazioni e pratiche del sacro. Atti dell'incontro internazionale di studi, Roma, Museo nazionale romano preistorico etnografico "Luigi Pigorini”, 20-21 maggio 2011, Roma 2012.

Anversa degli Abruzzi = G. Grossi (Ed.), Anversa degli Abruzzi. Historia e monumenta, S. Giovanni Teatino (CH) 2007.

Anxa-Angitia = A. D'Ercole - R. Simini - G. Grossi (Ed.), La città marsa di Anxa-Angitia. Un contributo per la realizzazione del Parco Archeologico, Pescara 1995.

Apex = G. Casadio - A. Mastrocinque - C. Santi (Ed.), Apex. Studi storico-religiosi in onore di Enrico Montanari, Roma 2016.

Approches épigraphiques $=\mathrm{S}$. Demougin - M. Navarro Caballero (Ed.), Se déplacer dans l'Empire romain. Approches épigraphiques. XVIII rencontre franco-italienne d'épigraphie du monde romain, Bordeaux, 7-8 octobre 2011, Paris 2014.

Arcella, Fasti = L. Arcella, Fasti. Il lavoro e la festa: note al calendario romano arcaico, Roma 1992.

Archaeologia Pisana = S. Bruni - T. CARUso - M. Massa (Ed.), Archaeologia Pisana. Scritti per Orlanda Pancrazzi (Terra Italia. Collana di studi archeologici sull'Italia antica 7), Pisa 2004.

Archeologia Cristiana VII = E. Russo (Ed.), 1983-1993: dieci anni di archeologia cristiana in Italia. Atti del VII Congresso Nazionale di Archeologia Cristiana, Cassino, 20-24 settembre 1993 I-III, Cassino 2003.

Archeologia Medievale VII = P. ARTHUR - M. L. IMPERIALE (Ed.), VII Congresso nazionale di archeologia medievale. Palazzo Turrisi, Lecce, 9-12 settembre 2015, Sesto Fiorentino (FI) 2015.

Aree montane $=\mathrm{S}$. MaGnani (Ed.), Le aree montane come frontiere. Spazi d'interazione e connettività. Atti del convegno internazionale, Udine, 10-12 dicembre 2009 (Studi di frontiera 1), Ariccia (RM) 2013.

Armellini, Antichi cimiteri $=$ M. Armellini, Gli antichi cimiteri cristiani di Roma e d'Italia, Roma 1893.

ARnaldi, Neptunus = A. ARNALDI, Ricerche storico-epigrafiche sul culto di Neptunus nell'Italia romana (Stud. Ist. It. Stor. Ant. 64), Roma 1997.

ARnolds, Funktionen = M. ARnolds, Funktionen republikanischer und frühkaiserzeitlicher Forumsbasiliken in Italien, Diss. Heidelberg 2005.

Arqueología = Arqueología de las ciudades modernas superpuestas a las antiguas, Madrid 1985.

Arrigoni Bertini, Parma romana $=$ M. G. Arrigoni BerTini, Parma romana. Contributo alla storia della città, Parma 2004.

Arrigoni Bertini, Simbolo $=$ M. G. Arrigoni Bertini, $I$ simbolo dell'ascia nella Cisalpina romana (Epigrafia e Antichità 24), Bologna - Faenza 2006.

Arte dall'Abruzzo $=$ S.O.S. Arte dall'Abruzzo. Una mostra per non dimenticare, Roma 2010.

Asinio Pollione = P. Domenicucci (Ed.), Asinio Pollione. Atti del Convegno Asinio Pollione e la gens Asinia tra Teate Marrucinorum e Roma, Chieti, 1 luglio 2011, Lanciano (CH) 2012

Aspects of friendship = M. PEACHIN (Ed.), Aspects of friendship in the Graeco-Roman world. Proceedings of a confe- 
rence held at the Seminar für Alte Geschichte, Heidelberg, on 10-11 June, 2000 (JRA. Supplementary Series 43), Portsmouth (RI) 2001.

Assisi e gli Umbri nell'antichità = G. BONAMENTE - F. COARELLI (Ed.), Assisi e gli Umbri nell'antichità. Atti del Convegno Internazionale, Assisi, 18-21 dicembre 1991, Assisi (PG) 1997.

Asta ac pellege = J. Del Hoyo - J. Gómez Pallarès (Ed.), Asta ac pellege. 50 años de la publicación de Inscripciones Hispanas en Verso, de S. Mariner (Signifer 8), Madrid 2002.

Atti città etrusca = Studi sulla città antica . Atti del Convegno di studi sulla città etrusca e italica preromana, Bologna 1970.

Atti Conv. Abr.-Mol. I-III = Atti e Memorie del Convegno Storico Abruzzese-Molisano. Casalbordino 1931, I-III, Casalbordino (CH) 1935.

Atti Conv. Beni Culturali $=$ Atti del I Convegno Regionale de Beni Culturali e Ambientali d'Abruzzo, Chieti 1981.

Atti Conv. Gruppi Archeologici I = Atti del I Convegno de Gruppi Archeologici dell'Italia Meridionale, Prata Sannita, 25-27 Aprile 1986, Roma 1988.

Atti del Terzo Congresso = Atti del Terzo Congresso internazionale di epigrafia greca e latina, Roma, 4-8 settembre 1957, Roma 1959.

Atti Roma I-II = Atti dell'XI Congresso Internazionale di Epigrafia Greca e Latina, Roma, 18-24 settembre 1997, I-II, Roma 1999.

Atti Tibullo = Atti del Convegno Internazionale di Studi su Albio Tibullo, Roma - Palestrina, 10-13 maggio 1984 (Supplemento di "Ciceroniana"), Roma 1986.

Aubert, Business Managers = J.-J. Aubert, Business Managers in Ancient Rome. A Social and Economic Study of Institores, 200 B. C. - A. D. 250 (Columb. Stud. Class. 21), Leiden - New York - Köln 1994.

Augenti, Lavoro schiavile $=$ D. Augenti, Il lavoro schiavile a Roma (Arti e mestieri nel mondo romano antico 3), Roma 2008.

Augustae = A. Kolb (Ed.), Augustae. Machtbewusste Franen am römischen Kaiserhof? Herrschaftsstrukturen und Herrschaftspraxis II. Akten der Tagung in Zürich 18.20.9.2008, Berlin 2010

Augusto = S. SEgENNI (Ed.), Augusto dopo il bimillenario. Un bilancio (Studi sul mondo antico 8), Milano 2018.

Aulo Virgio Marso = M. Malavolta et AL. (Ed.), Aulo Virgio Marso e il vicus Anninus nelle testimonianze epigrafiche e archeologiche. Atti del Convegno di Studi, Torre di Castel luccio - Lecce nei Marsi, 26 agosto 2006, Bella (PZ) 2008.

Aurigemma, Terme di Diocleziano ${ }^{6}=\mathrm{S}$. Aurigemma, Le Terme di Diocleziano e il Museo Nazionale Romano ${ }^{6}$ (Itinerari dei musei, gallerie e monumenti d'Italia 78), Roma 1970.

AusBüTtel, Untersuchungen = F. M. AusBüTTEL, Untersuchungen zu den Vereinen im Westen des römischen Reiches (Frankfurter Althistorische Studien 11), Kallmünz 1982.

Ausbüttel, Verwaltung = F. M. Ausbüttel, Die Verwaltung der Städte und Provinzen im spätantiken Italien (Europäische Hochschulschriften III 343), Frankfurt a. M. 1988.

Autocélébration = M. CÉBEILLAC-GERVASONI - L. LAMOINE F. Trément (Ed.), Autocélébration des élites locales dans le monde romain. Contexte, textes, images (II ${ }^{e}$ s. av. J.-C. $I I I^{e}$ s. ap. J.-C.) (Collection Erga 7), Rome - Clermont-Ferrand 2004

Autour de la notion de sacer $=$ TH. LANFRANCHI (Ed.), Autour de la notion de sacer (Coll. de l'École Fr. de Rome 541), Rome 2018.

Bacchielli, Parole d'oltremare $=$ L. Bacchielli, Parole d'oltremare e altri scritti di archeologia (Ludus philologiae 9), Urbino 2002
Bagnall - Cameron - Schwartz - Worp, Consuls = R. S. Bagnall - A. Cameron - S. R. SChWARTZ K. A. WORp, Consuls of the Later Roman Empire (Philol. Monogr. Amer. 36), Atlanta (GA) 1987.

Balzano, Aufidena = V. Balzano, Dove fu Aufidena, Castel di Sangro (AQ) 1899.

Balzano, Aufidena Caracenorum = V. Balzano, Aufidena Caracenorum ai confini settentrionali del Sannio. Memorie storiche intorno all'antichità di Castel di Sangro, Roma 1923.

Balzano, Castel di Sangro = V. Balzano, La vita di un comune del reame: Castel di Sangro, Pescara 1942 (lucis ope iterum impressum Castel di Sangro (AQ) 1985).

BALZANo, Inventario $=\mathrm{V}$. BALZANo, Inventario degli oggetti esistenti nel Museo Aufidenate di Castel di Sangro (libellus machina dactylographica a. 1949 scriptus in bibliotheca municipali vici Castel di Sangro sine inv. n. adservatus).

Barbieri, $A l b o=$ G. Barbieri, L'albo senatorio da Settimio Severo a Carino (193-285) (Stud. Ist. It. Stor. Ant. 6), Roma 1952.

BARBIERI, Scritti minori $=$ G. BARBIERI, Scritti minori. Raccolti per iniziativa della Scuola di Specializzazione in Archeologia dell'Università di Roma - "La Sapienza" (Vetera 3), Roma 1988.

Bartman, Portraits = E. Bartman, Portraits of Livia. Imaging the Imperial Woman in Augustan Rome, Cambridge 1999.

Bartolomeo Borghesi = Bartolomeo Borghesi: scienza e libertà. Colloquio internazionale AIEGL 1981 (Studi di Storia 1), Bologna 1982.

Basilica di Valva = La cattedrale basilica di Valva, Roma 1971.

Basilicata = M. Salvatore (Ed.), Basilicata. L'espansionismo romano nel sud-est d'Italia. Il quadro archeologico. Atti del convegno, Venosa, 23-25 aprile 1987, Venosa (PZ) 1990.

BAsilici, Chiesa di San Pietro = M. BAsilici, La chiesa di San Pietro in Pereto (AQ) (Quaderni del Museo Civico di Cerchio 127), Cerchio (AQ) 2011.

Basilici, Epigrafi $=$ M. BASILICI, Epigrafi romane in Pereto (Ouaderni del Museo Civico di Cerchio 123), Roma 2011.

BAYET, Hercule $=\mathrm{J}$. BAYET, Les origines de l'Hercule romains (Bibl. des Écoles Fr. d'Athènes et de Rome 132), Roma 1926.

Belgica et Italica $=\mathrm{J}$--CH. BALTY (Ed.), Belgica et Italica. Joseph Mertens: une vie pour l'archéologie. Alba in excelso locata saxo ... Obscura incultis Herdonia ab agris. Atti del Convegno in memoria di Joseph Mertens, Rome, Academia Belgica, 4-6 dicembre 2008 (Institut Historique Belge de Rome, Artes 2), Bruxelles - Brussel - Roma 2012.

Beloch, Römische Geschichte = J. BeLOCH, Römische Geschichte bis zum Beginn der punischen Kriege, Berlin Leipzig 1926.

Beltrán Lloris - Díaz Ariño, El nacimiento = F. Beltrán Lloris - B. Díaz Ariño (Ed.), El nacimiento de las culturas epigráficas en el occidente mediterráneo: modelos romanos y desarrollos locales (III-I a. E.), Madrid 2018.

Benelli - Rizzitelli, Culture funerarie = E. Benelli - C. Rizzitelli, Culture funerarie d'Abruzzo (IV-I secolo a. C.) (Mediterranea. Supplemento 5), Pisa - Roma 2010.

Beni culturali = E. CATANI (Ed.), I beni culturali di Fermo e territorio. Atti del Convegno, Fermo, Palazzo dei Priori, 1518 giugno 1994, Fermo 1996.

Berardinelli, Cenni = G. Berardinelli, Trivento (Chiesa vescovile), in V. D'Avino, Cenni storici sulle chiese arcivescovili, vescovili e prelatizie (nullius) del Regno delle Due Sicilie, Napoli 1848, 692-700 (editio altera: G. BERARDINELLI, Cenni storici sulla chiesa vescovile di Trivento, Trivento [CB] 2005).

Berti, ms. = Berti, Dissertazione sull'iscrizione di Marco Bebio del padre Alessandro Berti della Congregazione 
della Madre di Dio (liber manu scriptus a. 1723/25 in bibliotheca municipali Vastensi sine inv. n. servatus).

Bertolotto, Città antiche $=\mathrm{E}$. BertolotTo, Alcune città antiche intorno a Roma nella documentazione epigrafica del Museo Nazionale Romano (liber machina dactylographica redactus thesis doctoris dignitatis nominisque a. 1996/97 Romae impetratae apud Università degli Studi di Roma "La Sapienza", Scuola Nazionale di Archeologia).

Besnier, De regione Paelignorum $=\mathrm{M}$. BesNIER, De regione Paelignorum, Lutetiae Parisiorum 1902.

Betтi, $m s .=$ B. M. Betтi, Storia del Vasto (liber manu scriptus saec. XIX in bibliotheca communali Vastensi sine inv. n. servatus).

Bianchi Bandinelli - Giuliano, Etruschi e Italici $^{2}=\mathrm{R}$. Bianchi Bandinelli - A. Giuliano, Etruschi e Italici prima del dominio di Roma², Milano 1978.

BIELla, Bronzi votivi = M. C. BIELLA, I bronzi votivi dal santuario di Corfinio. Località Fonte Sant'Ippolito (Biblioteca di "Studi Etruschi" 54), Roma 2015.

Biferno = G. De BeneditTis (Ed.), Il porto romano sul Biferno tra storia e archeologia, Campobasso 2008.

Bignami, Bronzetti $=$ L. Bignami, I bronzetti di Valle Fuino di Cascia conservati nei Musei Vaticani, Spoleto (PG) 1987.

BINDI, Monumenti $=$ V. BINDI, Monumenti storici ed artistici degli Abruzzi con prefazione di F. Gregorovius, Napoli 1889.

Biographie = K. Vössing (Ed.), Biographie und Prosopographie. Internationales Kolloquium zum 65. Geburstag von Anthony R. Birley. 28. September 2002, Schlo $\beta$ Mickeln, Düsseldorf (Historia Einzelschriften 178), Stuttgart 2005.

A. R. BiRley, Fasti of Roman Britain = A. R. BIRLey, The Fasti of Roman Britain, Oxford 1981.

E. Birley, The Roman Army = E. Birley, The Roman Army: papers 1929-1986, Amsterdam 1988.

E. BiRley, Roman Britain and the Roman Army ${ }^{2}=$ E. BIRLEY, Roman Britain and the Roman Army. Collected Papers ${ }^{2}$, Kendal 1961

Bispham, Municipalization = E. Bispham, From Asculum to Actium. The Municipalization of Italy from the Social War to Augustus, Oxford 2007.

Bistoni - Bozzi, Norcia = U. Bistoni - F. Bozzi, Norcia, storia e storiografia di una città, Perugia 1983.

BÖLS-JANSSEN, La vie religieuse $=$ N. BöLS-JANSSEN, La vie religieuse des matrones dans la Rome archaïque (Coll. de l'École Fr. de Rome 176), Rome 1993.

BOLKESTEIN, Wohltätigkeit $=\mathrm{H}$. BOLKESTEIN, Wohltätigkeit und Armenpflege im vorchristlichen Altertum: ein Beitrag zum Problem "Moral und Gesellschaft", Utrecht 1939.

Bolli laterizi $=$ CHR. BruUn (Ed.), Interpretare $i$ bolli laterizi $d i$ Roma e della Valle del Tevere: produzione, storia economica e topografia. Atti del Convegno all'École Française de Rome e all'Institutum Romanum Finlandiae, 31 marzo e 1 aprile 2000, organizzato da CHr. BRUUn $e$ Fr. Chausson (Acta Inst. Rom. Finl. 32), Roma 2005.

Bollin, Antichità classiarie $=$ M. Bollini, Antichità classia rie, Longo (RA) 1968.

Bollmann, Vereinshäuser = B. Bollmann, Römische Vereins häuser. Untersuchungen zu den Scholae der römischen Berufs-, Kult- und Augustalen-Kollegien in Italien, Mainz 1998.

BONANNI - ZACCHIA, La pietra scritta $=$ E. BONANNI - A. ZACCHIA, La pietra scritta e l'alta Valle del Turano. Storia di un monumento $e$ di un confine tra equicoli e sabini in età romana, Città di Castello (PG) 1986.

BonaRIA, Mimorum Romanorum Fragmenta I-II = M. BonARIA, Mimorum Romanorum Fragmenta I-II (Pubblicazion dell'Istituto di Filologia Classica 5, 1-2), Genova 1955/56.
BONKOFFsKy, Curatores = V. BONKOFFSKY, Municipale curatores in Italie en de westelijke provincies tijdens het principat I-II, Gent 2002.

Borlenghi, Campus = A. Borlenghi, Il campus. Organizzazione e funzione di uno spazio pubblico in età romana. Le testimonianze in Italia e nelle Province occidentali (Thiasos 1), Roma 2011.

Boschung, Grabaltäre = D. Boschung, Antike Grabaltäre aus den Nekropolen Roms (Acta Bernensia 10), Bern 1987.

Bottiglioni, Manuale = G. BotTiglioni, Manuale dei dialetti italici (Osco, Umbro e dialetti minori). Grammatica, testi, glossario con note etimologiche, Bologna 1954.

Boulvert, Domestique = G. Boulvert, Domestique et fonctionnaire sous le Haut-Empire romain. La condition de l'affranchi et de l'esclave du prince (Annales Littéraires de l'Université de Besançon, Centre de Recherches d'Histoire Ancienne 9), Paris 1974.

Boulvert, Esclaves = G. Boulvert, Esclaves et affranchis impériaux sous le Haut-Empire romain. Rôle politique et administratif (Collection Labeo 4), Napoli 1970.

Bourdin, Les peuples = S. Bourdin, Les peuples de l'Italie préromaine: Identités, territoires et relations interethniques en Italie centrale et septentrionale (VIII $-I^{e r} s$. av. J.-C.) (Bibl. des Écoles Fr. d'Athènes et de Rome 350), Rome 2012.

Bove, S. Salvatore Telesino = E. Bove, S. Salvatore Telesino: da Casale a Comune, Piedimonte Matese (CE) 1990.

BracCeschi, ms. = I. B. Bracceschi, Commentarij per l'Historia di Spoleto (liber a. 1591/99 exaratus in Archivio Campello apud Sezione di Archivio di Stato di Spoleto servatus sine $\mathrm{n}$. inv.).

Brancato, Alumni $=$ N. G. Brancato, Una componente trasversale nella società romana: gli alumni. Inscriptiones Latinae ad alumnos pertinentes commentariumque, Roma 2015.

BRANCATO, Repertorium $=$ N. G. BRANCATO, Repertorium delle trasmissioni del gentilizio nel mondo romano sulla base della documentazione epigrafica I-II, Roma 2009/11.

BRANDT, Tod und Suizid = H. BRANDT, Am Ende des Lebens. Alter, Tod und Suizid in der Antike (Zetemata 136), München 2010.

BrÉLAz, Corpus = C. BRÉLAZ, Corpus des inscriptions grecques et latines de Philippes II.1 (Études épigraphique 6), Athènes 2014.

Bricault, Atlas = L. Bricault, Atlas de la diffusion des cultes isiaques (IV s. av. J.-C. $-I V^{e}$ s. apr. J.-C.) (Mém. Acad. Inscr. Bell. Lett. 23), Paris 2001.

BRICAULT, Recueil = L. BRICAULT, Recueil des inscriptions concernant les cultes isiaques (Mém. Acad. Inscr. Bell. Lett. 31), Paris 2005.

Brilliant, Gesture and Rank = R. Brilliant, Gesture and Rank in Roman Art (Mem. Conn. Acad. Art. Scien. 14), New Haven (CT) 1963.

Brique antique $=\mathrm{P}$. BOUCHERON $-\mathrm{H}$. BROISE $-\mathrm{Y}$. THÉBERT (Ed.), La brique antique et médiévale. Production et commercialisation d'un matériau. Actes du colloque international organisé par le Centre d'histoire urbaine de l'École normale supérieure de Fontenay/Saint-Cloud et l'École française de Rome (Saint-Cloud, 16-18 novembre 1995) (Coll. de l'École Fr. de Rome 272), Rome 2000.

BriQuel, Catalogue = D. BrIQuel, Catalogue des inscriptions étrusques et italiques du musée du Louvre, Paris 2016.

Briquel, Pélasges = D. Briquel, Les Pélasges en Italie. Recherches sur l'histoire de la légende (Bibl. des Écoles Fr. d'Athènes et de Rome 252), Paris - Rome 1984.

Britain and Rome = M. G. JARRETT - B. Dobson (Ed.), Britain and Rome. Essays presented to E. Birley on his Sixtieth Birthday, Kendal 1966. 
Broekaert, Navicularii $=\mathrm{W}$. Broekaert, Navicularii et Negotiantes. A prosopographical study of Roman merchants and shippers (Pharos. Studien zur griechisch-römischen Antike 28), Rahden 2013.

Brogi, $m s .=$ T. Brogi, Sul lago Fucino e sue escrescenze: progetti per bonificarlo colla descrizione dell'Emissario di Tiberio Claudio; e sulla necessità di riaprirlo (liber manu scriptus a. 1815/16 servatus Romae apud Biblioteca Angelica sub n. 1959 f. 345-360 olim p. 1-32, publici iuris factus ab A. M. Socciarelli, Cerchio (AQ) 2012 [vide ad nomen]).

Broughton, Magistrates $=$ T. R. S. Broughton, The Magistrates of the Roman Republic. With the Collaboration of M. L. Patterson (Philol. Monogr. Amer. 15. 1-3). I. 509 B. C. -100 B. C., New York 1951. II. 99 B. C. - 31 B. C., New York 1952. III. Supplement, Atlanta (GA) 1986.

Brouwer, Bona Dea $=$ H. H. J. Brouwer, Bona Dea. The Sources and a Description of the Cult (EPRO 110), Leiden 1989.

BRUHL, Liber Pater = A. BRUHL, Liber Pater. Origine et expansion $d u$ culte dionysiaque à Rome et dans le monde romaine (Bibl. des Écoles Fr. d'Athènes et de Rome 175), Paris 1953.

BrunetTI, Aprutii monumenta $=$ F. BRUnetTI, Sacra ac profana Aprutii monumenta: fol. 233: primum itinerarium cui ultima manus imposita mense decembris anni 1645 Campli, ed. Roberto Ricci (Delficina 3), Teramo 2000.

Bruno, I Sabini = M. G. Bruno, I Sabini e la loro lingua, Bologna 1969.

Bruns, Fontes = C. G. Bruns (Ed.), Fontes iuris Romani antiqui. Leges et negotia post curas Theodori Mommseni editionibus quintae et sextae adhibitas septimum edidit $\mathrm{O}$. Gradenwitz, Tubingae 1909.

BRuUn, Water Supply = CHR. BRuUn, Water Supply of Ancient Rome. A Study of Roman Imperial Administration (Soc. Scient. Fennica, Comment. Human. Litt. 93), Helsinki 1991.

Bucci, Studio = I. Bucci, Studio topografico del territorio compreso tra la Valle del Pescara e la Valle dell'Orta (Interpromium et Ceii) (Academia Historico-Iuridico-Theologica "Petrus Točanel". Studia et Documenta 12), Roman 2006.

Budischovsky, Diffusion = M.-CH. Budischovsky, La diffusion des cultes isiaques autour de la mer Adriatique I. Inscriptions et monuments (EPRO 61), Leiden 1977.

BüCHELER, Kleine Schriften = F. BüCHELER, Kleine Schriften IIII, Leipzig - Berlin 1915/30.

Buonocore, Abruzzo e Molise I-II = M. BuOnocore, L'Abruzzo e il Molise in età romana tra storia ed epigrafia (DASP. Studi e testi 21. 1-2), L'Aquila 2002.

Buonocore, Aesernia $=$ M. Buonocore, Molise. Repertorio delle iscrizioni latine. Aesernia (Istituto regionale per gli Studi Storici del Molise "V. Cuoco" V. 2), Campobasso 2003.

Buonocore, Appunti I-V = M. Buonocore, Appunti $d i$ topografia romana nei codici Lanciani della Biblioteca Apostolica Vaticana I-V, Roma 1997/2002 (vide infra LANCIANI, $m s$.).

Buonocore, Censimento $=$ M. Buonocore, Per un censi mento delle iscrizioni latine lapidarie del Sannio Settentrionale, Roma 1994.

Buonocore, Codices Vaticani $=$ M. Buonocore, Codices Vaticani Latini. Codices 9734-9782 (Codices Amatiani), Città del Vaticano 1988 (vide supra AmAтı, ms.).

BuONocore, Codici epigrafici $=$ M. BuONocore, Tra i codici epigrafici della Biblioteca Apostolica Vaticana (Epigrafia e Antichità 22), Bologna 2004.
BuONOCORE, Epigrafia anfiteatrale $=$ M. BuONOCoRE, Epigrafia anfiteatrale dell'Occidente romano III. Regiones Italiae II-V, Sicilia, Sardinia et Corsica (Vetera 6), Roma 1992.

BuONOCORE, Iscrizioni latine $=\mathrm{M}$. BuONOCORE, Le iscrizioni latine d'età romana murate nella Cattedrale Valvense a Corfinio (Museo delle genti d'Abruzzo 11), Pescara 1985.

BuONocore, Superaequum $=$ M. Buonocore, L'epigrafia latina di Superaequum. Corpus delle iscrizioni (Contr. Valle Peligna. Quaderno 4), Sulmona (AQ) 1985.

BuONocore, Tradizione manoscritta $=\mathrm{M}$. BuONocore, $\mathrm{La}$ tradizione manoscritta dell'epigrafia classica abruzzese nei codici della Biblioteca Apostolica Vaticana (DASP. Studi e testi 8), L’Aquila 1986.

Caballos Rufino, Bronce de Osuna = A. Caballos Rufino, El nuevo bronce de Osuna y la política colonizadora romana (Historia y Geografía 115), Sevilla 2006.

Cagnat, Cours $^{4}=\mathrm{R}$. Cagnat, Cours d'épigraphie latine ${ }^{4}$, Paris 1914.

Cainzza, Archeologia e storia = D. Cainzza, Archeologia e storia antica del mandamento di Pietramelara e del Montemaggiore I-II, Ancona 1995.

CAIAzza, Società e cultura $=\mathrm{D}$. CAIAzza, Società e cultura nell'Alta Terra di Lavoro del '700, Telese Terme (CE) 2014.

CAlabi Limentani, Epigrafia latina ${ }^{1}=$ I. Calabi Limentani, Epigrafia latina $^{1}$, Varese - Milano 1968.

Calabi Limentani, Epigrafia latina ${ }^{4}=$ I. Calabi Limentani, Epigrafia latina ${ }^{4}$, Milano - Modena 1991.

Calabi Limentani, Studi = I. Calabi Limentani, Studi sulla società romana. Il lavoro artistico (Biblioteca storica universitaria II. 9), Milano - Varese 1958.

Caldelli, Agon Capitolinus = M. L. Caldelli, L'agon Capitolinus. Storia e protagonisti dall'istituzione domizianea al IV secolo (Stud. Ist. It. Stor. Ant. 54), Roma 1993.

Calderini, Aquileia romana $=$ A. CALDERINI, Aquileia romana. Ricerche di storia e di epigrafia, Milano 1930.

Calore, Interpromium = P. L. Calore, in G. De Petra - P. L. CAlore, Interpromium e Cei, Atti Reale Accad. Arch. Napoli 21, 1900/01, 153-192.

Camodeca, Ceti dirigenti $=$ G. Camodeca, $I$ ceti dirigenti $d i$ rango senatorio equestre e decurionale della Campania romana I, Casoria (NA) 2008.

CAmodeca, Puteoli romana $=$ G. CAModeca, Puteoli romana: istituzioni e società. Saggi, Napoli 2018.

CAmpedelli, Amministrazione = C. CAMPedelli, L'amministrazione municipale delle strade romane in Italia (Antiquitas 1. 62), Bonn 2014.

Campi aperti = A. Clementi (Ed.), I campi aperti di Peltuinum dove tramonta il sole ... Saggi sulla terra di Prata d'Ansidonia dalla protostoria all'età moderna (Studi sulla storia del territorio 1), L'Aquila 2007.

Campochiaro = Campochiaro: potenzialità d'intervento sui Beni Culturali, Campobasso 1982.

Campomarino = G. DE BeneditTis (Ed.), Campomarino. La necropoli di Marinelle Vecchie. Campagne di scavo 20092010 (Considerazioni di storia ed archeologia. I quaderni 3), Campobasso 2013.

Canale Parola, $m s$. I = E. Canale Parola, Breviarium manu scriptum inter d. 3 m. Dec. a. 1888 et d. 15 m. Iul. a. 1889 servatum Romae apud Archivio Centrale dello Stato (EUR). MPI. Dir. Gen. AABBAA. II versamento, $1^{\circ}$ serie, b. 15, fasc. 251, cui titulus "Villavallelunga. Scoperte di antichità. 1888”, publici iuris factum a L. Luschi, Stud. Class. Orient. 42, 1992 (1994), 252-257.

Canale Parola, $m s$. II = E. Canale Parola, Breviarium manu scriptum d. 29 m. Dec. a. 1888 servatum Romae apud Archivio Centrale dello Stato (EUR). MPI. Dir. Gen. AABBAA. II versamento, $1^{\circ}$ serie, $b .10$, fasc. 150 , cui titulus "Monumenti Epigrafici di Carsoli". 
Canale Parola, $m s$. III = E. Canale Parola, Breviarium manu scriptum d. 29 m. Dec. a. 1888 servatum Romae apud Archivio Centrale dello Stato (EUR). MPI. Dir. Gen. AABBAA. II versamento, $1^{\circ}$ serie, b. 12, fasc. 198 , cui titulus "Monumenti Epigrafici di Carsoli".

Canale Parola, $m s$. IV = E. Canale Parola, Epistulae inter a. 1887 et a. 1889 manu scriptae servatae Romae apud Archivio Centrale dello Stato (EUR). MPI. Dir. Gen. AAB$B A A$. II versamento, $1^{\circ}$ serie.

Canosa I = M. Chelotti - R. Gaeta - V. Morizio - M. SiLVESTRINI (Ed.), Epigrafi romane di Canosa I (Documenti e studi 4), Bari 1985.

Canosa II = M. Chelotti - V. Morizio - M. Silvestrin (Ed.), Epigrafi romane di Canosa II (Documenti e studi 7. 2), Bari 1990

Cantarelli, Diocesi $=$ L. Cantarelli, La Diocesi italiciana da Diocleziano alla fine dell'impero occidentale, Roma 1903.

Capdeville, Volcanus = G. CAPdeville, Volcanus, Recherches comparatistes sur les origines du culte de Vulcan (Bibl. des Écoles Fr. d'Athènes et de Rome 288), Paris - Rome 1995.

Capestrano = G. Chiarizia - L. IAgnemma (Ed.), Capestrano nella Valle Tritana, L'Aquila 2015.

CAPINI, Venafrum = S. CAPINI, Molise. Repertorio delle iscrizioni latine. Venafrum (Istituto regionale per gli Studi Storici del Molise "V. Cuoco" 7), Campobasso 1999.

Capogrossi Colognesi, Persistenza $a$ L. Capogrossi ColoGNESI, Persistenza e innovazione nelle strutture territorial dell'Italia romana: l'ambiguità di una interpretazione storiografica e dei suoi modelli, Napoli 2002.

Capogrossi Colognesi, Ricerche $=$ L. CAPOgrossi ColoGNESI, Ricerche sulla struttura delle servitù d'acqua in diritto romano, Milano 1966.

Capogrossi Colognesi, Struttura I-II = L. Capogrossi Colognesi, La struttura della proprietà e la formazione dei "iura praediorum” nell'età repubblicana I-II, Milano 1969/76.

Cappelle dei Marsi = R. SABATini et AL., Cappelle dei Marsi, Roma 1990.

Caratteri e diffusione = E. CAMPANILE (Ed.), Caratteri e diffusione del latino in età arcaica (Testi linguistici 19), Pisa 1993.

Carletti, La diocesi di Amiterno = S. Carletti, La diocesi di Amiterno. Note storiche e archeologiche, Città del Vaticano 1976.

Carlsen, Vilici $=$ J. CARLSEn, Vilici and Roman Estate Managers until AD 284 (Anal. Rom. Inst. Dan. Supplementum 24), Roma 1995.

Carroccia, Buca = M. Carroccia, Contributo topografico per la individuazione di Buca, porto romano della Frentania, Campobasso 1991

Carroccia, Scritti vari $=$ M. CARroccia, Scritti vari di topografia antica (Molise - Abruzzo - Campania). Questioni di metodo, Roma 2006.

Carroll, Spirits of the Dead $=$ M. Carroll, Spirits of the Dead. Roman Funerary Commemoration in Westen Europe (Oxford Studies in Ancient Documents), Oxford 2006.

Carta archeologica = G. F. GAMURrini - A. Cozza - A. PAsqui - R. MAngarelli, Carta archeologica d'Italia (1881-1897). Materiali per l'Etruria e la Sabina (Forma Italiae. Serie II. Documenti 1), Firenze 1972.

Carta archeologica IV = L. Quilici - S. Quilici Gigli (Ed.), Carta archeologica e ricerche in Campania IV. Comuni di Amorosi, Faicchio, Puglianello, San Salvatore Telesino, Telese Terme (ATTA. Supplemento 15. 4), Roma 2010.

Carta archeologica VII = L. QuilicI - S. Quilici Gigli (Ed.), Carta archeologica e ricerche in Campania VII. Comuni di
Ailano, Casapesenna, Castelvenere, Pratella, Raviscanina, Roccarainola, San Cipriano d'Aversa (ATTA. Supplemento 15. 7), Roma 2012.

Casali d'Aschi = G. Grossi - L. Palozzi - V. Amendola - A MelChiorre (Ed.), Casali d'Aschi ieri e oggi (Studi e testi 11), Roma 1983.

CAStAgnoli, Lavinium I = F. CASTAGNOLI, Lavinium I. Topografia generale, fonti e storia delle ricerche, Roma 1972.

Castagnoli, Topografia antica I-II = F. Castagnoli, Topografia antica. Un metodo di studio I. Roma. II. L'Italia, Roma 1993.

Castel di Ieri = A. Campanelli (Ed.), Il tempio di Castel di Ieri, Sulmona (AQ) 2007.

Castellani, Epigrafi albensi = C. Castellani, Epigrafi albensi (Appendix libri, cui nomen Vicende politiche $e$ caratteri istituzionali, civili e religiosi di Alba Fucens. Ricerche storico-epigrafiche machina dactylographica redacti thesis doctoris dignitatis nominisque a. 1977/78 Romae impetratae apud Facoltà di Magistero), Avezzano (AQ) 1982.

Castellani, Museo $=$ C. Castellani, Il museo lapidario di Avezzano (Appendix libri, cui nomen Vicende politiche e caratteri istituzionali, civili e religiosi di Alba Fucens. Ricerche storico-epigrafiche machina dactylographica redacti thesis doctoris dignitatis nominisque a. 1977/78 Romae impetratae apud Facoltà di Magistero), Avezzano (AQ) 1979.

Castrén, Ordo populusque Pompeianus = P. Castrén, Ordo populusque Pompeianus. Polity and Society in Roman Pompeii (Acta Inst. Rom. Finl. 8), Roma 1975.

Catalli, Museo Lapidario = F. Catalli, Il Museo Lapidario Comunale, Avezzano (AQ) 1998.

Catalogo Mostra Augustea = Catalogo della Mostra Augustea della Romanità, Roma 1937.

Cavallaro, Spese e spettacoli $=$ M. A. Cavallaro, Spese e spettacoli. Aspetti economici-strutturali degli spettacoli nella Roma giulio-claudia (Antiquitas 1. 34), Bonn 1984.

CCCA I-VII = M. V. Vermaseren (Ed.), Corpus cultus Cybelae Attidisque I-VII (EPRO 50. 1-7), Leiden 1987/89.

CÉBeIllac, Quaestores = M. CÉBEIllaC, Les "quaestores principis et candidati" aux $I^{\text {er }}$ et II ème siècles de l'Empire (CeSDIR. Monografie a supplemento degli "Atti" 4), Milano 1972.

Cébeillac-Gervasoni, Magistrats = M. CéBeillaC-GervaSONI, Les magistrats des cités italiennes de la seconde guerre punique à Auguste: le Latium et la Campanie (Bibl. des Écoles Fr. d'Athènes et de Rome 299), Rome 1998.

Cébeillac-Gervasoni - Caldelli - Zevi, Épigraphie latine = M. Cébeillac-Gervasoni - M. L. Caldelli - F. Zevi, Épigraphie latine, Paris 2006 (editio nova addendis corrigendisque augmentata Epigrafia latina. Ostia: cento iscrizioni in contesto, Roma 2010).

Cecconi, Governo imperiale = G. A. Cecconi, Governo imperiale e élites dirigenti nell'Italia tardoantica. Problemi di storia politico-amministrativa (270-476 d. C.) (Biblioteca di Athenaeum 24), Como 1994.

Celidonio, La diocesi di Valva = G. Celidonio, La diocesi di Valva e Sulmona I. Le origini cristiane, Casalbordino (CH) 1909. II. Dal 492 al 1100, Casalbordino (CH) 1910.

Celidonio, Memorie = G. Celidonio, Memorie storiche di Scanno (ms. a. 1911 servatus Sulmone penes Michaelem Celidonio, quem nunc publici iuris fecerunt G. Morelli et P. Caranfa, Memorie storiche di Scanno, Teramo 2017).

CENERINI, Donna romana $=\mathrm{F}$. CENERINI, La donna romana Modelli e realtà (Universale Paperbacks. Il Mulino 435), Bologna 2002

Centri storici = G. ChIARIzIA (Ed.), Centri storici della Val Pescara dall'Evo Medio ai nostri giorni, Pescara 1990. 
Cerasani, Annotazioni = E. Cerasani, Annotazioni epigrafiche marse esistenti nel CIL apud Sir Henry Colt HoAre, Viaggio classico attraverso l'Italia. Escursione da Roma al lago di Celano, \&c. in Abbruzzo I [traduzione e note a cura di E. Cerasani], Sulmona (AQ) 1982.

Cerasani, Marruvium = E. Cerasani, Marruvium e S. Sabina, Pratola Peligna (AQ) 1986.

Cesi, Commentarium = P. Cesi, Commentarium Rerum Cas siae, Fuligno 1655

Cesi, Eulogium = P. CESI, Eulogium de Cassiae antiquitate et aedificatione, Fulginiae 1655.

Ceti medi $=$ A. SARTori - A. Valvo (Ed.), Ceti medi in Cisalpina. Atti del Colloquio internazionale, Milano, 14-16 settembre 2000, Milano 2002.

Champeaux, Fortuna I-II = J. Champeaux, Fortuna. Recherches sur le culte de la Fortune à Rome et dans le monde romain des origines à la morte de César I-II (Coll. de l'École Fr. de Rome 64, 1-2), Rome 1982/87.

CHAMPlin, Final Judgments = E. Champlin, Final Judgments. Duty and Emotion in Roman Wills 200 B.C.E. - C.E. 250, Berkeley 1991.

Chantraine, Freigelassene $=$ H. Chantraine, Freigelassene und Sklaven im Dienst der römischen Kaiser. Studien zu ibrer Nomenklatur (Forsch. Sklaverei 1), Wiesbaden 1967.

Chastagnol, Pouvoir impérial = A. Chastagnol, Le pouvoir impérial à Rome. Figures et commémorations. Textes édités par St. Benoist et S. Demougin (Scripta varia 4. Hautes études du monde gréco-romain 41), Genève 2008.

Chausson, Stemmata aurea $=$ Fr. Chausson, Stemmata aurea. Constantin, Justine, Théodose. Revendications généalogiques et idéologie impériale au IV siècle ap. J.-C. (CRDAC 26), Rome 2007.

Chieti = U. De Luca (Ed.), Chieti e la sua provincia I. Storia, arte, cultura, Chieti 1990

Chioffi, Antium = L. Chioffi, Antium. Noterelle Antiatinae, Marina Anzio (RM) 2018.

Chioffi, Caro = L. Chioffi, Caro: il mercato della carne nell'Occidente romano (ATTA. Supplemento 4), Roma 1999.

Chioffi, Museo = L. Chioffi, Museo Provinciale Campano di Capua. La raccolta epigrafica. Le iscrizioni latine: cortili, sale, depositi. Con la collaborazione di L. Capurso $e$ M. Foglia (Museo Provinciale Campano. Cataloghi epigrafici 1), Capua (CE) 2005.

ChiovitTi, $m s .=$ B. ChiovitTi, Adversaria inter a. 1849 et 1877 manu scripta nunc apud gentem Bovianensem Marucci-De Fabritiis adservata et publici iuris facta a G. De Benedittis, Conoscenze 3, 1986, 67-94.

Chisari, Guardiola = G. Chisari, Il Castello di Guardiola nella terra delle Plaie, [s. 1.] 2014.

Cholodniak, Carmina sepulcralia ${ }^{2}=\mathrm{I}$. Cholodniak, Carmina sepulcralia Latina epigraphica ${ }^{2}$, Petropoli 1904.

Ciabarra, Anfiteatri = C. Ciabarra, Gli anfiteatri dell'Abruzzo romano: testi e contesti (liber machina dactylographica redactus thesis doctoris dignitatis nominisque a. 2015/16 Romae impetratae apud "Sapienza" Università di Roma).

Cianfarani, Culture adriatiche $=$ V. Cianfarani, Culture adriatiche d'Italia. Antichità tra Piceno e Sannio prima dei Romani, Roma 1970.

Cianfarani, Guida $=$ V. Cianfarani, Guida alle antichità di Sepino, Milano 1958

Cianfarani, Santuari nel Sannio = V. Cianfarani, Santuari nel Sannio, Pescara 1960

Cianfarani, Schede I = V. Cianfarani, Schede del Museo Nazionale di Chieti. Terza serie, Chieti 1972.

Cianfarani, Schede II = V. Cianfarani, Schede del Museo Nazionale di Chieti. Quarta serie, Chieti 1973.
Cianfarani, Terra italica $=$ V. Cianfarani, Terra italica, Torino 1959.

Cicala, Instrumentum = G. Cicala, Instrumentum domesticum inscriptum proveniente da Asculum e dal suo territorio (Bibl. Stud. Antichi 91), Pisa 2010.

Cicerone, Tussio = G. Cicerone, Tussio nei 99 castelli fondatori dell'Aquila degli Abruzzi, Roma 1939.

Ciceroniana = Ciceroniana . Atti del X Colloquium Tullianum, Monte Sant'Angelo, 24-27 aprile 1997, Roma 1998.

Cifarelli, Tra Lazio e Campania = F. M. Cifarelli, Tra Lazio e Campania. Ricerche di Storia e di Topografia antica (Università degli Studi di Salerno. Quaderni del Dipartimento di Scienze dell'Antichità 16. Serie Storia Antica e Archeologia 2), Napoli 1995.

CIG = A. BовCKH et AL., Corpus Inscriptionum Graecarum IIV, Berolini 1828/77.

CIIud = J.-B. Frey (Ed.), Corpus Inscriptionum Iudaicarum. Recueil des inscriptions juives qui vont $d u I I I^{e}$ siècle avant Jésus-Christ au VII siècle de notre ère I. Europe (Sussidi allo studio delle antichità cristiane 1), Roma 1936.

CILAnd = Corpus de Inscripciones Latinas de Andalucía.

Cirelli, Il regno delle due Sicilie = F. Cirelli, Il regno delle due Sicilie descritto e illustrato, Napoli 1853/60.

Cités de l'Italie $=$ M. Ghilardi - Chr.-J. Goddard $-\mathrm{P}$. Porena (Ed.), Les cités de l'Italie tardo-antique (IV $-V I^{e}$ siècle). Institution, économie, société, culture et religion (Coll. de l'École Fr. de Rome 369), Paris - Rome 2006.

Città campane = G. VITOLO (Ed.), Le città campane fra tarda Antichità e alto Medioevo. Atti del Seminario (Napoli, 2004), Salerno 2005.

Ciucci, ms. I = F. CiuccI, Vetusta Nursia. I-II: Historie [dell'antica città] di Norsia dove si tratta della sua Sibilla, e dei Laghi (liber a. 1650 fortasse exaratus in Biblioteca Nazionale di Napoli adhuc servatus sub n. IX. B. 38-39. Agitur de recensione, quam Mommsen appellat priorem).

CiuccI, $m s$. II = F. CiuccI, Historie dell'antica Città di Norsia dove si tratta della sua Sibilla e dei Laghi (liber a. 1650 exaratus, quem Mommsen excussit Nursiae apud heredes viri Feliciani Patrizi-Forti; ubi autem nunc exstet, ignotum est; excludendum non est, quin hic liber idem ac manu scriptus n. V eiusdem Ciucci, de quo vide infra. Agitur de recensione, quam MommSEN appellat alteram).

Ciucci, $m s$. III = F. Ciucci, Vetusta Nursia. I-II: Istorie dell'antica Città di Norsia dove si tratta della sua fondazione, dell'antiche Famiglie, d'alcuni fatti d'arme con suoi vicini Popoli, della vita de' Santi, degl'Imperadori, e Capitani, delli suoi Castelli, della Grotta della Sibilla, e delli suoi maravigliosi Laghi (liber saec. XVIII manu scriptus ex exemplari a. 1653 depromptus in Biblioteca Giovardiana oppidi Veroli servatus sub n. 42. 2.10).

Ciucci, $m s$. IV = F. Ciucci, Istorie dell'antica città di Norsia dove si tratta della sua Sibilla e dei Laghi (liber saec. XVIII manu scriptus ex exemplari a. 1653 depromptus Spoletii servatus olim apud comites Pila nunc in Biblioteca arcivescovile 'G. M. Mastai Ferretti' sub Fondo Don Ansato Fabbi sine inv. n. [nunc vide G. Ceccarelli - C. Comino (Ed.), Istorie dell'antica Città di Norsia, Firenze 2003, 112-355]).

CIUCCI, $m s$. V = F. CIUCCI, Vetusta Nursia. Istorie dell'antica Città di Norsia dove si tratta della sua Sibilla, e dei Laghi (liber temporis spatio inter saec. XVIII ex. et saec. XIX in. comprehenso manu scriptus ex exemplari a. 1650 depromptus, qui in archivo communali Nursino sine inv. $n$. exstabat; postquam terrae motus d. $24 \mathrm{~m}$. Aug. et d. $30 \mathrm{~m}$. Oct. a. 2016 universum oppidum diruerunt, Spoleto est translatus; excludendum non est, quin hic liber idem ac manu scriptus n. II eiusdem CiUccI, de quo vide supra).

Civiltà dei Romani = S. SETTIS (Ed.), Civiltà dei Romani [III]. Il rito e la vita privata, Milano 1992. 
Civitas Penne I = L. Franchi dell'Orto - C. Vultaggio (Ed.), Civitas Penne. La città medievale (Storia e civiltà di Penne 3), Roma 2015.

Civitas Penne II = L. Franchi dell'Orto - C. Vultaggio (Ed.), Civitas Penne. Il Medioevo delle arti (Storia e civiltà di Penne 4), Roma 2015.

Clark, Divine Qualities = A. J. Clark, Divine Qualities. Cult and Community in Republican Rome, Oxford 2007.

Clauss, Cultores Mithrae $=$ M. Clauss, Cultores Mithrae. Die Anhängerschaft des Mithras-Kultes (HABES 10), Stuttgart 1992.

Clauss, Kaiser und Gott = M. Clauss, Kaiser und Gott. Herr scherkult im römischen Reich, Stuttgart 1999.

Clauss, Mithras = M. Clauss, Mithras. Kult und Mysterien, München 1990.

Clauss, Untersuchungen $=$ M. Clauss, Untersuchungen $z u$ den principales des römischen Heeres von Augustus bis Diokletian. Cornicularii, speculatores, frumentarii, Bochum 1973.

CLE = F. BüCHELER (Ed.), Carmina Latina epigraphica I-II (Anthologia Latina sive poesis Latinae supplementum ediderunt $\mathrm{F}$. BüCHELER et A. Riese II 1-2), Lipsiae 1895/97.

CLE Suppl. = E. Lommatzsch (Ed.), Carmina Latina epigraphica III (Anthologia Latina sive poesis Latinae supplementum ediderunt $\mathrm{F}$. BüCHELER et A. RIESE II 3), Lipsiae 1926.

Clementi, Amiternum = A. Clementi, Amiternum dopo la distruzione. Elaborazione cartografica di C. Tовіа (DASP. Studi e testi 27), L'Aquila 2003.

CMC = A. VINCENT (Ed.), Corpus épigraphique des musiciens civils, Rome 2016 [https://filemaker.efrome.it/fmi/ webd\#CMC] (etiam vide infra VINCENT, Jouer pour la cité).

CMM = A. VINCENT (Ed.), Corpus épigraphique des musiciens militaires, Rome 2016 [https://filemaker.efrome.it/fmi/ webd\#CMM] (etiam vide infra VINCENT, Jouer pour la cité).

CoArelli, Foro Romano = F. CoArelli, Il Foro Romano I. Periodo arcaico. II. Periodo repubblicano e augusteo (Lectiones Planetariae 1-2), Roma 1985/86.

Coarelli, Lazio = F. Coarelli, Lazio (Guide Archeologiche Laterza 5), Roma - Bari 1982.

Coarelli - La Regina, Abruzzo e Molise = F. CoArelli - A. La Regina, Abruzzo e Molise (Guide Archeologiche Laterza 9), Roma - Bari 1984, ed. altera 1993.

Coccia, $m s .=$ F. Coccia, Piano di Recupero n. 32 / Norcia Centro 1 [Roma post 1979], 13 / par. 7.0-7.2 (liber manu scriptus in bibliotheca communali Nursina sine inv. n. olim servatus; postquam terrae motus d. $24 \mathrm{~m}$. Aug. et d. $30 \mathrm{~m}$. Oct. a. 2016 universum oppidum diruerunt, Spoleto est translatus).

Colantoni, ms. I = L. Colantoni, Epistula manu scripta $\mathrm{d}$. 25 m. Febr. a. 1884 ad Rosato Sclocchi missa nunc servata in Archivio di Stato civitatis L'Aquila.

Colantoni, $m s$. II = L. Colantoni, Epistula manu scripta $\mathrm{d}$. 7 m. Mai. a. 1884 ad Giuseppe Fiorelli missa nunc servata Romae apud Archivio Centrale dello Stato. (EUR). MPI. Dir. Gen. AABBAA. Posizione "Aquila", prot. n. 5987.

Colantoni, $m s$. III = L. Colantoni, Epistulae manu scriptae inter a. 1888 et a. 1897 nunc servatae Romae apud Archivio Centrale dello Stato (EUR). MPI. Dir. Gen. AABBAA. II versamento, $1^{\circ}$ serie, b. 12, fasc. 200.

Colantoni, Storia dei Marsi = L. Colantoni, Storia dei Marsi dai tempi più antichi fino alla guerra Marsica, Italica o Sociale, Lanciano $(\mathrm{CH}) 1889$.

Colarieti Tosti, Villa $=$ G. Colarieti Tosti, $\mathrm{La}$ villa d'Assio nella Campagna Romana, Rieti 1904.
Colarossi Mancini, Memorie storiche $=$ A. Colarossi Mancini, Memorie storiche di Popoli fino all'abolizione dei feudi, Popoli 1911.

Colarossi Mancini, Storia di Scanno = A. Colarossi ManCINI, Storia di Scanno e guida nella Valle del Sagittario, L'Aquila 1921.

Colasanti, Pinna $=$ G. Colasanti, Pinna. Ricerche di topografia e di storia, Roma 1907.

Colasanti, Reate $=$ G. Colasanti, Reate. Ricerche di topografia medievale e antica, Perugia 1910 (lucis ope iterum impressum Reate a. 1995 curavit T. LegGIO).

Colella, Inventarium ms. = N. Colella, Inventarium titulorum musei municipalis Corfiniensis (liber a. 1903 manu scriptus; servatur Romae apud Archivio Centrale dello Stato. MPI. Dir. Gen. AABBAA. Antichità e Scavi. II versamento. $1^{\circ}$ serie, $b .13$, fasc. 221 ).

Colini, Il fascio littorio = A. M. Colini, Il fascio littorio, Roma 1933.

Collezione Torlonia = A. CAmpanelli (Ed.), La Collezione Torlonia di antichità del Fucino, Sambuceto (CH) 2003.

Colonie e municipi $=$ G. PACI - S. M. MARengo - S. AntolinI (Ed.), Colonie e municipi nell'era digitale. Documentazione epigrafica per la conoscenza delle città antiche. Atti del Convegno di studi, Macerata, 10-12 dicembre 2015 (Ichnia 14), Tivoli (RM) 2017.

Colons et colonies $=$ S. Demougin - J. SCheId (Ed.), Colons et colonies dans le monde romain. Actes de la XV Rencontre franco-italienne sur l'épigraphie du monde romain (Paris, 4-6 octobre 2008) (Coll. de l'École Fr. de Rome 456), Rome 2012.

Colosi - Costantini, Sabina Tiberina $=$ F. Colosi - A. Costantini, La Sabina Tiberina in epoca romana. Ricognizioni nel territorio tra Otricoli e Magliano Sabina (Arte, Architettura, Tecnologie 1), Roma 2017.

Comunicazione = M. G. Angeli Bertinelli - A. Donati (Ed.), La comunicazione nella storia antica. Fantasie e realtà. Atti del III Incontro Internazionale di Storia Antica (Genova, 23-24 settembre 2006) (Serta antiqua 11), Roma 2008.

Comunità indigene $=\mathrm{J}$. Mertens $-\mathrm{R}$. LAmbrechts (Ed.), Comunità indigene e problemi della romanizzazione nell'Italia centro-meridionale (IV-III sec. av. C.). Actes $d u$ Colloque International organisé à l'occasion du $50^{e}$ anniversaire de l'Academia Belgica et du $40^{e}$ des fouilles belges en Italie, Rome, Academia Belgica, $1^{\text {er }}$-3 février 1990 (Inst. Hist. Belge de Rome, Études 29), Bruxelles - Rome 1991.

Confine nel tempo = R. Ricci - A. Anselmi (Ed.), Il confine nel tempo. Atti del Convegno, Ancarano, 22-24 maggio 2000, L'Aquila 2005.

Conoscenza = A. Di Iorio (Ed.), Per la conoscenza dell'Antico Sannio. Giornata di studio tenuta ad Isernia il 6 giugno 1992 (Quad. Conoscenza 14), Roma 1993.

Convegno Virgiliano $=$ Atti del Convegno Virgiliano di Brindisi nel Bimillenario della morte, Brindisi, 15-18 ottobre 1981, Perugia 1983.

Conventi, Città romane = M. Conventi, Città romane di fondazione (Studia archaeologica 130), Roma 2004.

Conway, Italic Dialects = R. S. Conway, The Italic Dialects I, Cambridge 1897.

Corazza, Augustales = G. Corazza, Gli Augustales della Campania romana, Napoli 2016.

Corbier, Aerarium = M. Corbier, L'Aerarium Saturni et l'aerarium militare. Administration et prosopographie sénatoriale (Coll. de l'École Fr. de Rome 24), Paris - Roma 1974.

CORBIER, Donner à voir $=\mathrm{M}$. CORBIER, Donner à voir, donner à lire. Mémoire et communication dans la Rome ancienne, Paris 2006. 
Corcia, Storia delle due Sicilie = N. Corcia, Storia delle due Sicilie dall'antichità più remota al 1789 I, Napoli 1843.

Cordella, Norcia $=$ R. Cordella, Norcia e territorio, Norcia (PG) 1995.

Cordella - Criniti, Ager Nursinus = R. CoRdella - N. CRINiti, Ager Nursinus. Storia, epigrafia e territorio di Norcia e della Valnerina romane (Bibl. Dep. Umbria 2), Perugia 2008.

Cordella - Criniti, Iscrizioni $=$ R. CORdella - N. Criniti, Iscrizioni latine di Norcia e dintorni (Quaderni di "Spoletium” 1), Spoleto (PG) 1982.

Cordella - Criniti, Nuove iscrizioni = R. Cordella - N. Crinitr, Nuove iscrizioni latine di Norcia, Cascia e Valnerina (Quaderni di "Spoletium” 5), Spoleto (PG) 1988.

Cordella - Criniti, Sabina settentrionale $=$ R. Cordella N. CRINITI, Parole su pietre. Epigrafia e storia nella Sabina settentrionale di età romana (Bibl. Dep. Umbria 15), Perugia 2014.

Corolla Epigraphica = C. Deroux (Ed.), Corolla Epigraphica. Hommages au professeur Yves Burnand (Collection Latomus 331), Bruxelles 2011.

Corsignani $=$ M. BuOnocore - G. Morelli (Ed.), Pietro Antonio Corsignani nel terzo centenario della nascita (1686-1986). Atti del Convegno di studi, Celano, 8-9 novembre 1986, L'Aquila 1987.

Costarella - Isabella, Tre iscrizioni imperiali $=\mathrm{A}$. Costa RELla - C. IsabelLa, Tre iscrizioni imperiali di I e III secolo ed una epigrafe sepolcrale di I secolo d. C. nella città di Piedimonte Matese, Piedimonte Matese (CE) 2005.

Costarella - Prisco, Museo Civico = A. Costarella - R. Prisco, Il Museo Civico di Piedimonte nei Documenti dell'Archivio Storico, Piedimonte Matese (CE) 2004.

Cottanello = M. Sternini (Ed.), La villa di Cottanello (Bibl. Arch. 8), Bari 2000.

Courtney, Musa Lapidaria = E. Courtney, Musa Lapidaria A Selection of Latin Verse Inscriptions (American Philological Association. American Classical Studies 36), Atlanta (GA) 1995

Cresci Marrone - Mennella, Pisaurum = G. Cresci MarRONe - G. Mennella, Pisaurum I. Le iscrizioni della colonia (Bibl. Stud. Antichi 44), Pisa 1984.

Crespo Ortiz de ZÁrate, Nutrices I-II = S. Crespo Ortiz DE ZÁrate, Nutrices en el imperio Romano. I: Estudio de las fuentes y prosopografia, Valladolid 2005; II: Estudios social, Zaragoza 2006.

Cristofori, Non arma virumque ${ }^{2}=$ A. Cristofori, Non arma virumque. Le occupazioni nell'epigrafia del Piceno ${ }^{2}$ (Tarsie. Studi di antichistica 2), Bologna 2004.

Cryptoportiques $=$ Les cryptoportiques dans l'architecture romaine. Actes du Colloque, 19-23 avril 1972 (Coll. de l'École Fr. de Rome 14), Paris - Rome 1973.

Cugusi, Aspetti letterari ${ }^{2}=$ P. Cugusi, Aspetti letterari dei Carmina Latina epigraphica ${ }^{2}$ (Testi e manuali 22), Bologna 1996.

Cugusi, Carmina Latina Sardiniae $=$ P. CugusI, Carmina Latina Epigraphica Provinciae Sardiniae (Testi e manuali 74), Bologna 2003.

Cugusi - Sblendorio Cugusi, Carmina Latina Africana $=\mathrm{P}$ Cugusi - M. T. Sblendorio Cugusi, Carmina Latina Africarum provinciarum post Buechelerianam collectionem editam reperta cognita (CLEAfr) (Epigrafia e Antichità 34), Bologna - Faenza 2014.

Cugusi - Sblendorio Cugusi, Carmina Latina Dalmatiae = P. Cugusi - M. T. Sblendorio Cugusi, Carmina Latina Epigraphica non-bücheleriani di Dalmazia (CLEDalm). Edizione e commento con osservazioni sui carmi bücheleriani della provincia (Epigrafia e Antichità 36), Bologna Faenza 2015
Cugusi - Sblendorio Cugusi, Carmina Latina Hispanica = P. Cugusi - M. T. Sblendorio Cugusi, Carmina Latina Epigraphica Hispanica post Buechelerianam collectionem editam reperta cognita (CLEHisp) (Epigrafia e Antichità 31), Bologna - Faenza 2012

Cugusi - Sblendorio Cugusi, Carmina Latina Moesica Thraciae $=$ P. Cugusi - M. T. Sblendorio Cugusi, Carmina Latina Epigraphica Moesica (CLEMoes). Carmina Latina Epigraphica Thraciae (CLEThr) (Testi e manuali 104), Bologna 2008

Cugusi - Sblendorio Cugusi, Carmina Latina Pannonica = P. Cugusi - M. T. Sblendorio Cugusi, Carmina Latina Epigraphica Pannonica (CLEPann) (Testi e manuali 96), Bologna 2007.

Cugusi - Sblendorio Cugusi, Carmina Latina province africane $=$ P. Cugusi - M. T. Sblendorio Cugusi, Carmina Latina Epigraphica non-bücheleriani delle province africane. Introduzione al tema, materiali preparatori, edizione di testi, aspetti e problemi (Testi e manuali 126), Bologna 2012.

Cugusi - Sblendorio Cugusi, Versi su pietra $=$ P. Cugusi M. T. Sblendorio Cugusi, Versi su pietra. Studi sui Carmina Latina Epigraphica. Metodologia, problemi, tematiche, rapporti con gli auctores, aspetti filologici e linguistici, edizioni di testi. Quaranta anni di ricerche (Epigrafia e Antichità 38, 1-3), Bologna - Faenza 2016.

Cultes polythéistes $=$ Chr. Delplace - F. Tassaux (Ed.), Les cultes polythéistes dans l'Adriatique romaine. Séminaire Bordeaux 1997 (Ausonius publications. Études 4), Bordeaux 2000 .

Culti e religiosità $=\mathrm{L}$. ZerbinI (Ed.), Culti e religiosità nelle province danubiane. Atti del II Convegno internazionale, Ferrara, 20-22 novembre 2013 (Pubblicazioni del LAD 2), Bologna 2015.

Cults of Isis = V. GASPARINI - R. Veymiers (Ed.), Individuals and Materials in the Greco-Roman Cults of Isis. Agents, Images, and Practices I-II (Religions in the Graeco-Roman World 187), Leiden - Boston 2018.

Cultura della transumanza = E. NARCISO (Ed.), La cultura della transumanza. Atti del Convegno promosso dal Comune di Santa Croce del Sannio, dall'Istituto Storico "Giuseppe Maria Galanti" e dalla Comunità Montana "Alto Tammaro", 12-13 novembre 1988, Napoli 1991.

Cultura in Cesare = D. Poli (Ed.), La cultura in Cesare. Atti del Convegno Internazionale di Studi, Macerata - Matelica, 30 aprile - 4 maggio 1990 (Quaderni linguistici e filologici 5), Roma 1993.

Cultura italica $=$ La cultura italica. Atti del Convegno della Società Italiana di Glottologia, Pisa, 19-20 dicembre 1977, Pisa 1978.

Culture adriatiche antiche $=\mathrm{V}$. CIANFARANI $-\mathrm{L}$. FranchI Dell'Orto - A. La Regina, Culture adriatiche antiche di Abruzzo e Molise, Roma 1978.

Cultus splendore = A. M. CORDA (Ed.), Cultus splendore. Studi in onore di Giovanna Sotgiu, Senorbì (CA) 2003.

Cumae $=$ G. De Benedittis (Ed.), Cumae. Le Conferenze del Premio “E. T. Salmon” III, Campobasso 2000.

Cumont, Textes I-II = F. Cumont, Textes et monuments figurés relatifs aux Mystères de Mithra I-II, Bruxelles 1896/99.

Cuomo - Pellegrino, Monte Pallano = L. Cuomo - A. PelLEgrino, Il problema di Monte Pallano (Documenti 8), Roma 1976.

Curae cittadine = M. G. GRANINO CeCERE (Ed.), Le curae cittadine nell'Italia romana. Atti del Convegno, Siena, 18-19 aprile 2016 (Urbana species 4), Roma 2017.

Cures = M. CAVALIERI (Ed.), Cures tra archeologia e storia. Ricerche e considerazioni sulla capitale dei Sabini ed il suo territorio (Fervet opus 3), Louvain-la-Neuve 2017. 
D'Abrizio, Vittorito = M. D'Abrizio, Vittorito. Storia, tradi zione, folklore, Sulmona (AQ) 2001.

Da Camunni a romani $=\mathrm{S}$. Solano (Ed.), Da Camunni a romani. Archeologia e storia della romanizzazione alpina. Atti del Convegno, Breno - Cividate Camuno (BS), 10-11 ottobre 2013, Roma 2016.

Dai confini del mondo = A. Dionisio - S. Mari - R. TUTERI (Ed.), Dai confini del mondo alla patria di Ovidio. Merci, uomini e idee. Mostra archeologica, Sulmona [2015].

Dall'antica Histonium = A. R. STAFFA (Ed.), Dall'antica Histonium al Castello di Vasto, Vasto (CH) 1995.

Dalla villa di Ovidio = E. Matriocco (Ed.), Dalla villa di Ovidio al santuario di Ercole, Sulmona (AQ) 1989.

D'Amato, Proscingamento = S. D'Amato, Il primo proscingamento del Fucino. Gli scopi, le vicende e $i$ risultati della grande impresa di Roma, Avezzano (AQ) 1980.

D'ANELli, Histonium ed il Vasto = V. D'ANelli, Histonium ed il Vasto, Vasto (CH) 1977.

Daniel, Agrippa $=$ R. Daniel, M. Vipsanius Agrippa. Eine Monographie, Breslau 1933.

Darling Buck, Grammar = C. Darling Buck, A Grammar of Oscan and Umbrian. With a collection of inscriptions and a glossary, Boston 1904 (lucis ope iterum impressum Hildesheim - New York 1974).

D'Arpizio $m s .=$ V. D'Arpizio, Triginta tres fasciculi signati 1.1-13.1 et 1.2-20.2 servati San Benedetto dei Marsi in bibliotheca communali, qui complectuntur breviaria, adversaria, collectanea, opuscula ad Marruvium plerumque pertinentia inter a. 1940-1975 a ludi magistro VINCENZo D'ArPizio (1893-1977) redacta.

DAvid, Appariteurs = J.-P. DAvid, Au service de l'honneur. Les appariteurs de magistrats Romains (Mondes anciens 7), Paris 2019.

DAY, Architectural Terminology = B. DAy, The Architectural Terminology of the Ancient Roman Theatre in the West: An Epigraphic Approach (liber machina dactylographica redactus thesis doctoris dignitatis nominisque a. 1998 impetratae apud University Hamilton).

De Baroni Bonanni, Relazione $=$ F. DE Baroni Bonanni, Relazione dei lavori eseguiti nell'Archivio Provinciale del $2^{\circ}$ Abruzzo Ulteriore dal $1^{\circ}$ Agosto al 31 Luglio 1879 giusta le Ministeriali Istruzioni del 4 Aprile 1843, Pescara 1879.

DE Benedictis, $m s .=$ G. DE Benedictis, Memorie istoriche del Vasto antiche e moderne del sacerdote GIUSEPPE DE BenEDictis del Vasto (liber manu scriptus saec. XVIII in BENEDICTIS del Vasto (liber manu scriptus saec. XV
bibliotheca communali Vastensi sine inv. n. servatus).

De Benedittis, Bovianum = G. De Benedittis, Molise . Repertorio delle iscrizioni latine. Bovianum (Istituto regionale per gli Studi Storici del Molise "V. Cuoco" I), Campobasso 1995.

De Benedittis, Bovianum ed il suo territorio $=\mathrm{G}$. De BeneDitTis, Bovianum ed il suo territorio. Primi appunti di topografia storica (Documenti 7), Salerno 1977.

De Benedittis, Fagifulae $=$ G. De Benedittis, Molise. Repertorio delle iscrizioni latine. Fagifulae (Istituto regionale per gli Studi Storici del Molise "V. Cuoco" III), Campobasso 1997.

De Benedittis, Provincia $=$ G. De Benedittis, La Provincia Samnii e la viabilità romana (con la collaborazione di $\mathrm{D}$. Cainzza) (Quaderni dell'Associazione 4), Cerro al Volturno (IS) 2010.

De Benedittis, Saepinum = G. De Benedittis, Echi del Sannio. Guida alle antichità di Saepinum [supplementum ad n. VII, 2014, ephemeridis Considerazioni di Storia ed Archeologia], Campobasso 2016.

De Benedittis - GaggiotTi - Matteini Chiari, Saepinum =
ChIARI, Saepinum - Sepino. Guida turistico-culturale, Verona 1984.

De Benedittis - Matteini Chiari - Terzani, Territorio = G. De Benedittis - M. Matteini Chiari - G. Terzani, Molise. Repertorio delle iscrizioni latine. Il territorio e la città di Aesernia (Istituto regionale per gli Studi Storici del Molise "V. Cuoco" V. 1), Campobasso 1999.

De Carlo, Ceto equestre $=\mathrm{A}$. De Carlo, Il ceto equestre di Campania, Apulia et Calabria, Lucania et Bruttii dalla tarda Repubblica al IV secolo I-II (Vetera 19), Roma 2015.

De Chiara, Origini = G. De ChIARA, Origini e monumenti della città di Chieti, Chieti 1857 (ed. anastatica Bologna 1977).

DE Ciocchis, Agnone = R. DE Ciocchis, Insediamenti di epoche diverse in località S. Lorenzo di Agnone (Collana Altomolisana 5), Isernia 2007.

Dediche sacre = J. Bodel - M. Kajava (Ed.), Dediche sacre nel mondo greco-romano. Diffusione, funzioni, tipologie. Religious Dedications in the Greco-Roman World. Distribution, Typology, Use. Institutum Romanum Finlandiae, American Academy in Rome, 19-20 aprile 2006 (Acta Inst. Rom. Finl. 35), Roma 2009.

De Francesco, Ricerche $=$ D. De Francesco, Ricerche sui villaggi nel Lazio dall'età imperiale alla tarda antichità, Roma 2014.

Degli Abbati, Da Roma a Solmona = L. Degli Abbati, Da Roma a Solmona. Guida storico-artistica delle regioni attraversate dalla strada ferrata, Roma 1888 [= Guida storico-artistica delle regioni attraversate dalla strada ferrata con 53 illustrazioni. Rielaborazione di Adelmo Polla. Presentazione di Vincenzo Lucarelli (I Tascabili d'Abruzzo 150), Cerchio (AQ) 2004].

Degrassi, Fasti $=$ A. Degrassi, I Fasti consolari dell'Impero Romano dal 30 avanti Cristo al 613 dopo Cristo (SL Sussidi eruditi 3), Roma 1952.

DEGRASSI, Imagines = A. DEGRASSI, Inscriptiones Latinae liberae rei publicae. Imagines (CIL Auctarium), Berolini 1965.

Degrassi, Scritti vari I-IV = A. Degrassi, Scritti vari di antichità raccolti da amici e allievi nel $75^{\circ}$ compleanno dell'autore I-II. Roma 1962. III. Venezia - Trieste 1967. IV. Trieste 1971

DE Guidobaldi, Iscrizione arcaica $=$ D. DE GuIdobaldi, Intorno una iscrizione arcaica di $T$. Vezio con ricerche su l'Ercole Giovio e sul brato od erba sabina e sua natura, e simbolica, Napoli 1864.

De LaurentiIs, Iscrizioni $=$ C. De Laurentis, Iscrizioni italiche e romane (liber manu scriptus saec. XX in. in bibliotheca provinciali oppidi Chieti servatus sign. n. VIII, 2).

Del Chicca, Frontino = F. Del Chicca, Frontino. De aquae ductu urbis Romae. Introduzione, testo critico, traduzione e commento, Roma 2004.

Delehaye, Les origines $d u$ culte $^{2}=\mathrm{H}$. Delehaye, Les origines $d u$ culte des martyres ${ }^{2}$ (Subsidia hagiographica 20), Bruxelles 1933.

L. Del Giudice, Chiesa di S. Vincenzo = L. Del Giudice, La chiesa di S. Vincenzo di Saragozza o della Madonna delle Rose in Carsoli (AQ). Indagini archeologiche sul sito (I Quaderni di Lumen 57), Pietrasecca (AQ) 2012.

S. Del Giudice, Memorie istoriche $=$ S. Dei GiUdice, Memorie istoriche, antiche e moderne, sacre e profane de' Popoli Marrucini e di Chieti loro Metropoli, raccolte da Autori, da manoscritti ed altri monumenti (liber manu scriptus saec. XVIII in bibliotheca provinciali oppidi Chieti servatus sign. n. $X)$.

Delplace, Romanisation $=$ Chr. Delplace, La romanisation du Picenum. L'exemple d'Urbs Salvia (Coll. de l'École Fr. de Rome 177), Rome 1993.

Del Ponte, Riproduzioni = R. Del Ponte, Riproduzioni di antiche iscrizioni di Chieti e del territorio marrucino (liber 
manu scriptus saec. XIX post medium in bibliotheca provinciali oppidi Chieti servatus sign. n. LXXX, 6).

De Luca, Pagine di storia $=$ T. De Luca, Pagine di storia frentana e di Ortona antica, Ortona (CH) 1979.

DemircioĞLu, Der Gott auf dem Stier = H. DemircioĞlu, Der Gott auf dem Stier. Geschichte eines religiösen Bildtypus, Berlin 1939.

Demougin, L'ordre équestre $=\mathrm{S}$. Demougin, L'ordre équestre sous les Julio-Claudiens (Coll. de l'École Fr. de Rome 108), Paris - Roma 1988

Demougin, Prosopographie $=$ S. Demougin, Prosopographie des chevaliers romains julio-claudiens (43 av. J.-C. -70 ap. J.-C.) (Coll. de l'École Fr. de Rome 153), Paris - Roma 1992.

DE NINO, Lettere $=$ A. De NINO, Lettere a Ivan Vladimirovic Cuetaev (1880-1906), ed. G. PAPPONETTI - E. CeCCARONI (Contr. Valle Peligna. Quaderno 14), Sulmona (AQ) 2002.

De Nino, $m s .=$ A. De Nino, Libri manu scripti saec. XIX ex. - XX in. servati Romae apud Archivio Centrale dello Stato. MPI. Dir. Gen. AABBAA. Antichità e Scavi (vide BuONoCORE, Suppl. It. 3, 1987, 94; Eund., ibid. 4, 1988, 12; EUND., ibid. 5, 1989, 87).

De Nino, Saggio archeologico = A. De Nino, Saggio archeologico sulla ubicazione di alcuni oppidi, pagi e vici, Sulmona (AQ) 1905

De Nino, Scoperte archeologiche $=$ A. DE Nino, Scoperte archeologiche comunicate all'Accademia Nazionale dei Lincei e pubblicate nelle Notizie degli Scavi di Antichità, ed. E. Matтiocco (DASP. Studi e testi 23), L'Aquila 2002.

D'Eredità, Aveia = V. M. GiovinAzzi, Della città di Aveja ne' Vestini. Introduzione, revisione e note a cura di ANTONIO D'Eredità, Pescara 2006 [id est translatio in linguam Italicam hodiernam libri V. M. Giovenazzi, Della città di Aveia ne Vestini ed altri luoghi di antica memoria, Roma 1773, cum adnotationibus A. D'EREditè] (etiam cf. A. Cappelli, RASLA 19, 1904, 291-298).

DE Robertis, Contributi = F. M. DE Robertis, Contributi alla storia delle corporazioni a Roma, Bari 1933.

De Robertis, Diritto associativo = F. M. DE Robertis, Il diritto associativo romano. Dai collegi della Repubblica alle corporazioni del Basso Impero, Bari 1938.

De Robertis, Il fenomeno associativo ${ }^{2}=$ F. M. DE Robertis, Il fenomeno associativo nel mondo romano. Dai collegi della Repubblica alle corporazioni del Basso Impero ${ }^{2}$, Roma 1981.

De Robertis, Scritti varii $=$ F. M. DE Robertis, Scritti varii di diritto romano I-III, Bari 1987.

DE Robertis, Storia delle corporazioni = F. M. DE Robertis, Storia delle corporazioni e del regime associativo nel mondo romano I-II, Bari s. a. [circ. 1971].

De Roma a las provincias = A. CABAllos Rufino - E. MeLCHOR GIL (Ed.), De Roma a las provincias. Las elites como instrumento de proyección de Roma (Historia y Geografía 287), Sevilla 2014.

De Rosalia, Iscrizioni latine arcaiche ${ }^{2}=\mathrm{A}$. De Rosalia, Iscrizioni latine arcaiche (Hermes. Collana di testi antichi 8), editio altera correcta et aucta indicibus locorum et verborum instructa Palermo 1978.

DE Rossi, $m s .=$ G. B. DE Rossi, Libri manu scripti saec. XIX in Bibliotheca Apostolica Vaticana adservati sign. Vaticani Latini 10512-10543 (etiam cf. codices sign. Vat. lat. 14238-14298).

De Ruggiero - Vaglieri, Sylloge epigraphica $=$ E. De RugGIERO, Sylloge epigraphica orbis Romani. Vol. 2, pars 1: Inscriptiones Italiae regionum I-V, ed. D. VAGLIERI, Romae 1892/94 (editio altera lucis ope perfecta Romae
C. De Ruyt, Macellum = C. De Ruyt, Macellum. Marché alimentaire des Romains (Publications Louvain 35), Louvain 1983.

F. De Ruyt, Alba Fucens = F. De Ruyt, Alba Fucens III. Sculptures d'Alba Fucens (pierre, marbre, bronze): catalogue raisonné (Inst. Hist. Belge de Rome. Études 21), Bruxelles Rome 1982.

F. De Ruyt, Scripta Minora = F. De Ruyt, Scripta Minora (Publications Louvain 5), Louvain 1975.

De Salvo, Economia privata = L. De Salvo, Economia privata e pubblici servizi nell'Impero romano. I corpora naviculariorum (Kleiò 5), Messina 1992.

De Santis, Ager Aequiculanus = A. De SAntis, L'ager Aequiculanus. Analisi storico-epigrafica (liber machina dactylographica redactus thesis doctoris dignitatis nominisque a. 2008/09 Romae impetratae apud Università degli Studi di Roma "La Sapienza").

De Sisto, Raviscanina = A. De Sisto, Raviscanina, paese mio, Ercolano (NA) 1988.

Destephanis, $m s .=$ F. Destephanis, Delle Chiese, e sagre reliquie, ch'esisteno (liber manu scriptus inter saec. XVIII ex. et a. 1814 in aedibus privatis Sulmone servatus. Gratias ago Ezio Matтiocco, qui me certiorem fecit).

Devaluations = Les "Devaluations" à Rome. Époque républicaine et impériale I. [colloque] Rome, 13-15 novembre 1975 (Coll. de l'École Fr. de Rome 37, 1), Rome 1978. II. [colloque] Gdańsk, 19-21 octobre 1978 (Coll. de l'École Fr. de Rome 37, 2), Rome 1980.

Devijver Equestrian Officers I = H. Devijver, The Equestrian Officers of the Roman Imperial Army (Mavors 6), Amsterdam 1989.

Devijver Equestrian Officers II = H. Devijver, The Equestrian Officers of the Roman Imperial Army (Mavors 9), Stuttgart 1992.

D'Henry - Terzani, Manufatti lapidei = G. D'Henry - C. Terzani, Manufatti lapidei da Aesernia, Campobasso 1997.

Di Benedetto, Sulmona = A. Di Benedetto, Storia civile di Sulmona. Dal municipio romano alla città medievale (secc. I-XIII), Sulmona (AQ) 1990.

Di BIASE, Alba Fucens = C. Di BIASE, Alba Fucens. La documentazione epigrafica dopo il Corpus Inscriptionum Latinarum (liber machina dactylographica redactus thesis doctoris dignitatis nominisque a. 1999/2000 Romae impetratae apud Università degli Studi di Roma "La Sapienza").

Di Cecco Di Marino, Farantíca ${ }^{2}$ = G. Di Cecco Di Marino, Farantíca. Profilo storico, documentazione e foto antiche di Fara San Martino ${ }^{2}$, Lanciano (CH) 2004.

Di Cosmo, Iscrizione funeraria = L. Di Cosmo, Un'iscrizione funeraria rinvenuta nel territorio di S. Angelo d'Alife, Sant'Angelo d'Alife (CE) 1983 (separatum expressum ex IV Convegno dei Gruppi Archeologici della Campania, Afragola, 23-24 aprile 1983).

DI Costanzo, $m s .=$ G. G. DI Costanzo, Viaggio antiquario <saec. XVIII ex.>. Nell'Umbria e luoghi limitrofi. Nel Lazio e Sabina. Presso $i$ Vestini, i Marsi, Sannio e Campania. Manoscritto dell'Abate GIUS $<$ EPP $>$ E GIUstino DI Costanzo (liber primis annis saec. XIX a Luigi Belmonti O.S.B. exaratus in Bibliotheca Apostolica Vaticana adservatus sign. Vat. lat. 10544).

Diebner, Aesernia $=$ S. Diebner, Aesernia - Venafrum. Untersuchungen $z u$ den römischen Steindenkmälern zweier Landstädte Mittelitaliens (Archaeologica 8), Roma 1979.

DieHL, Altlateinische Inschriften ${ }^{5}=\mathrm{E}$. DieHL, Altlateinische Inschriften ${ }^{5}$ (Kleine Texte 38/40), Berlin 1964.

DieHL, Vulgärlateinische Inschriften = E. DieHL, Vulgärlateinische Inschriften (Kleine Texte 62), Bonn 1910.

Di Fazio, Feronia = M. Di Fazio, Feronia. Spazi e tempi di una dea dell'Italia centrale antica, Roma 2013. 
Di Iorio, Antologia = A. Di IorIo, Antologia di Bovianum Vetus. Memorie dal 1840 al 1939 (Quad. Conoscenza 51), Roma 2005.

Di Iorio, Bovianum Vetus = A. Di IorIo, Bovianum Vetus oggi Pietrabbondante. Ricerche storico-archeologiche con presentazione di GiUseppe PoRTo, Roma 1974.

Di Iorio, Epistolario = A. Di Iorio, Gli scavi di Bovianum Vetus e il Duca di Pescolanciano Giovanni Maria D'Alessandro. Epistolario inedito 1857-1860 (Quad. Conoscenza 8), Roma 1991

Di Iorio, Pietrabbondante ${ }^{2}=$ A. Di IorIo, Pietrabbondante Bovianum Vetus. Breve guida alla zona archeologica ${ }^{2}$, Roma 1985.

Di Iorio, Polverone $=$ A. Di Iorio, Bovianum Vetus ... ed il polverone, Roma 1975.

Di Iorio, Sannio = A. Di Iorio, Il Sannio nella storia. Il caso di Bovianum Vetus, Poggibonsi (SI) 2016.

Di Iorio, Scavi = A. Di Iorio, Gli scavi di Bovianum Vetus. Guida alla zona archeologica. Terza edizione illustrata, Roma 1998.

Di IORIO, Scritti vari I-II = A. DI IORIO, Scritti vari I-II, Roma 2003.

Di Lello, Conversando $=$ R. Di Lello, Conversando sulla cipolla in Alife, Piedimonte Matese (CE) 2014.

Di Niro, Culto di Ercole = A. Di Niro, Il culto di Ercole fra $i$ Sanniti Pentri e Frentani (Documenti 9), Roma 1977.

Di Niro - Pontarelli - Vaccaro, Canneto = A. Di Niro N. Pontarelli - D. Vaccaro, Canneto. Villa romana, Isernia 1995.

Dionisio, La Valle del Sagittario = A. Dionisio, La Valle del Sagittario e la conca peligna, Abruzzo, tra il IV e il I secolo a.C. Dinamiche e sviluppi della romanizzazione, Oxford 2015.

Diosono, Collegia $=$ F. Diosono, Collegia. Le associazioni professionali nel mondo romano (Arti e mestieri del mondo romano 1), Roma 2007.

Diotallevi, ms. = G. Diotallevi, Memorie Parrocchiali (liber manu scriptus ad a. 1872-1927 pertinens in archivo parochiali oppidi Savelli sine inv. n. adservatus).

D'IsAnto, Capua romana = G. D'IsAnto, Capua romana. Ricerche di prosopografia e storia sociale (Vetera 9), Roma 1993.

Di Stefano Manzella, Ambulacrum Iulianum = I. Di Stefano Manzella, Index Inscriptionum Musei Vaticani I. Ambulacrum Iulianum sive "Galleria Lapidaria" (ISS 1), Roma 1995.

Di Stefano Manzella, Mestiere di epigrafista $=$ I. Di STefano Manzella, Mestiere di epigrafista. Guida alla schedatura del materiale epigrafico lapideo (Vetera 1), Roma 1987.

Dituri, Territorio = C. Dituri, Dal Gran Sasso all'Aterno: il territorio fra Calascio e Barisciano nell'antichità (libellus machina dactylographica redactus thesis doctoris dignitatis nominisque a. 2007/08 L'Aquila impetratae apud Università degli Studi).

Dово́, Inscriptiones ${ }^{4}=$ А. Dовó, Inscriptiones extra fines Pannoniae Daciaeque repertae ad res earundem provinciarum pertinentes ${ }^{4}$, Budapestini 1975.

Dово́, Verwaltung = A. Dово́, Die Verwaltung der römischen Provinz Pannonien von Augustus bis Diocletianus: die provinziale Verwaltung, Budapest 1968.

Dobson, Primipilares $=$ B. Dobson, Die Primipilares. Entwicklung und Bedeutung, Laufbahnen und Persönlichkeiten eines römischen Offiziersranges (Beihefte der Bonner Jahrb. 37), Köln - Bonn 1978.

Documenta antiquitatis = G. ZANETTO - M. Ornaghi (Ed.), Documenta antiquitatis. Atti dei Seminari di Dipartimento 2009 (Quaderni di Acme 120), Milano 2010.
Domaszewski - Dobson, Rangordnung ${ }^{2}=$ A. von DomASZEWSKI, Die Rangordnung des römischen Heeres. 2. durchgesehene Auflage. Einführung, Berichtigungen und Nachträge von B. DoBson (Beihefte der Bonner Jahrb. 14), Köln - Graz 1967.

Domenico de Guidobaldi = G. Maretti (Ed.), La figura e l'opera di Domenico de Guidobaldi dai Borbone ai Savoia a duecento anni dalla nascita (1811-1902). Convegno di studi, Teramo - Nereto, 7-8 ottobre 2011, Colonnella (TE) 2014.

Domum tuam dilexi $=$ Domum tuam dilexi. Miscellanea in onore di Aldo Nestori (Stud. Ant. Crist. 53), Città del Vaticano 1998.

Donahue, Roman Community = J. F. Donahue, The Roman Community at Table during the Principate, Ann Arbor 2004.

Donati - Stefanetti, Dies natalis $=$ N. Donati - P. StefaNETTI, Dies natalis. I calendari romani e gli anniversari dei culti, Roma 2006.

Dondin-Payre, Acilii Glabriones = M. Dondin-Payre, Exercice du pouvoir et continuité gentilice. Les Acilii Glabriones du III siècle av. J.-C au Ve siècle ap. J.-C. (Coll. de l'École Fr. de Rome 180), Rome 1993.

Donna e lavoro = A. Buonopane - F. Cennerini (Ed.), Donna e lavoro nella documentazione epigrafica. Atti del I Seminario sulla condizione femminile nella documentazione epigrafica, Bologna, 21 novembre 2001 (Epigrafia e Antichità 19), Bologna - Faenza 2003.

Donna e vita cittadina = A. BuONopANe - F. CENERINI (Ed.), Donna e vita cittadina nella documentazione epigrafica. Atti del II Seminario sulla condizione femminile nella documentazione epigrafica, Verona, 25-27 marzo 2004 (Epigrafia e Antichità 23), Bologna - Faenza 2005.

Dorcey, Silvanus = P. F. Dorcey, The Cult of Silvanus. A Study in Roman Folk Religion (Columb. Stud. Class. 20), Leiden - New York - Köln 1992.

$D S=$ Dictionnaire des antiquités greques et romaines d'après les textes et les monuments. Ouvrage rédigé par une société d'écrivains spéciaux, d'archéologues et de professeurs sous la direction de mm. Ch. Daremberg et Edm. SAglio, Paris 1877/1919.

Ducroux, Catalogue = S. Ducroux, Catalogue analytique des inscriptions latines sur pierre conservées au Musée $d u$ Louvre, Paris 1975.

Dunbabin, Roman Banquet = K. M. D. Dunbabin, The Roman Banquet. Images of Conviviality, Cambridge 2003.

Duncan-Jones, Economy ${ }^{2}=$ R. Duncan-Jones, The Economy of the Roman Empire ${ }^{2}$, Cambridge 1982.

Dupraz, Vestins = E. Dupraz, Les Vestins à l'époque tardorépublicaine. Du nord-osque au latin, Mont-Saint-Aignan 2010.

DURRY, Cohortes prétoriennes $=\mathrm{M}$. DURRY, Les cohortes prétoriennes (Bibl. des Écoles Fr. d'Athènes et de Rome 146), Paris 1938.

Duthoy, Taurobolium = R. DuTHOY, The Taurobolium. Its Evolution and Terminology (EPRO 10), Leiden 1969.

Earthquakes $=$ E. GUIDOBonI - A. CoMAstri - G. Traina (Ed.), Catalogue of ancient earthquakes in the Mediterranean area up to the 10th century, Bologna 1994.

EcK, Epigrafia = W. EсK, Tra epigrafia, prosopografia e archeologia. Scritti scelti, rielaborati ed aggiornati (Vetera 10), Roma 1996.

Eck, L'Italia nell'impero romano $=\mathrm{W}$. EcK, L'Italia nell'impero romano. Stato e amministrazione in epoca imperiale (Documenti e studi 25), Bari 1999 (editio Italica correcta et aucta libri Eck, Organisation).

EcK, Organisation $=\mathrm{W}$. EcK, Die staatliche Organisation in der hohen Kaiserzeit (Vestigia 28), München 1979. 
EcK, Scritti scelti = W. EcK, Tra epigrafia, prosopografia e archeologia: scritti scelti, rielaborati ed aggiornati, Roma 1996.

EсK, Senatoren $=$ W. Eск, Senatoren von Vespasian bis Hadrian. Prosopographische Untersuchungen mit Einschlu $\beta$ der Jahres- und Provinzialfasten der Statthalter (Vestigia 13), München 1970.

Еск, Statthalter $=$ W. Еск, Die Statthalter der germanischen Provinzen vom 1.-3. Jahrbundert (Epigr. Studien 14), Köln - Bonn 1985.

EcK, Verwaltung $=\mathrm{W}$. EcK, Die Verwaltung des römischen Reiches in der hohen Kaiserzeit. Ausgewählte und erweiterte Beiträge 1-2 (Arbeiten zur römischen Epigraphik und Altertumskunde 1. 3), Basel - Berlin 1995/98.

ECK - Heinrichs, Sklaven und Freigelassene = W. Eck - J. HeInRICHS, Sklaven und Freigelassene in der Gesellschaft der römischen Kaiserzeit (Texte zur Forschung 61), Darmstadt 1993.

Écriture = M.-L. HAACK (Ed.), L'écriture et l'espace de la mort. Épigraphie et nécropoles à l'époque préromaine (Coll. de l'École Fr. de Rome 502), Rome 2016.

Écriture dans la maison = M. CORBIER - J.-P. GuILHEMBET (Ed.), L'écriture dans la maison romaine, Paris 2011.

EDER, Servitus publica $=$ W. EDER, Servitus publica. Untersuchungen zur Entstehung, Entwicklung und Funktion der öffentlichen Sklaverei in Rom (Forsch. Sklaverei 13), Wiesbaden 1980.

Effetto Alba Fucens = A. CAmpanelli (Ed.), Effetto Alba Fucens. Rivive la piccola Roma d'Abruzzo, Ascoli Piceno 2002.

Egypte - Grèce - Rome = Egypte - Grèce - Rome. Les différents visages des femmes antiques. Travaux et colloques du séminaire d'épigraphie grecque et latine de l'IASA, 2002/06 (Echo 7), Bern 2008.

Енмig, Donum dedit = U. Енмig, Donum dedit. . Charakteristika einer Widmungsformel in lateinischen Sakralinschriften (Pietas 9), Gutenberg 2017.

Ehmig - Haensch, LIA = U. Ehmig - R. Haensch, Die lateinischen Inschriften aus Albanien (LIA), Bonn 2012.

Ehrenberg - Jones, Documents ${ }^{2}=$ V. Ehrenberg A. H. M. JonEs, Documents Illustrating the Reigns of Augustus and Tiberius ${ }^{2}$, repr. with addenda, Oxford 1976.

EISNER, Grabbauten = M. EISNER, Zur Typologie der Grabbauten im Suburbium (RM, Ergänzungsheft 26), Mainz 1986.

Elenco = Elenco del fondo privato de' duchi D'Alessandro di Pescolanciano (liber manu scriptus servatus Aeserniae in Archivio di Stato di Isernia, sign. S.S. 40 n. 20, a. $1857 / 60$ exaratus).

Élites et leurs facettes = M. CÉBeILLAC-GERvASONI - L. LAMOINE (Ed.), Les élites et leurs facettes. Les élites locales dans le monde hellénistique et romain (Coll. de l'École Fr. de Rome 309. Collection Erga 3), Rome - Clermont-Ferrand 2003.

Élites municipales = M. CÉBEILLAC-GERVASONI (Ed.), Les élites municipales de l'Italie péninsulaire de la mort de César à la mort de Domitien entre continuité et rupture. Classes sociales dirigeantes et pouvoir central (Coll. de l'École Fr. de Rome 271), Paris - Rome 2000.

ENGESSER, Stadtpatronat $=$ L. ENGESSER, Der Stadtpatronat in Italien und den Westprovinzen bis Diokletian, Diss. Freiburg 1957.

Engfer, Private Munifizenz = K. ENGFER, Die private Munifizenz der römischen Oberschicht in Mittel- und Süditalien. Eine Untersuchung lateinischer Inschriften unter dem Aspekt der Fürsorge (Philippika. Altertumskundliche Abhandlungen 110), Wiesbaden 2017.
EngströM, Carmina = E. EngströM, Carmina Latina Epigraphica post editam collectionem Buechelerianam in lucem prolata, Gotoburgi - Lipsiae 1911.

Epigrafi Assisi = G. ForNI (Ed.), Epigrafi lapidarie romane di Assisi (Catalogo regionale dei beni culturali dell'Umbria [1]), Perugia 1987.

Epigrafia 2006 I-III = M. L. CAldelli - G. L. Gregori - S. Orlandi (Ed.), Epigrafia 2006. Atti della XIV Rencontre sur l'épigraphie in onore di Silvio Panciera con altri contributi di colleghi, allievi e collaboratori I-III (Tituli 9), Roma 2008.

Epigrafia dei Marsi = C. LetTA - S. D'Amato, Epigrafia della regione dei Marsi (CeSDIR. Monografie a supplemento degli "Atti" 7), Milano 1975.

Epigrafia dei porti = C. ZACCARIA (Ed.), L'epigrafia dei porti. Atti della XVII Rencontre sur l'epigraphie du monde romain (Ant. Alt. 79), Trieste 2014.

Epigrafia del villaggio $=$ A. Calbi - A. Donati - G. Poma (Ed.), L'epigrafia del villaggio. Atti del Colloquio AIEGL - Borghesi 1990, Forli, 27-30 settembre 1990 (Epigrafia e Antichità 12), Bologna - Faenza 1993.

Epigrafia della produzione = C. Nicolet - S. PANCIERA (Ed.), Epigrafia della produzione e della distribuzione. Actes de la $V I I e^{e}$ Rencontre franco-italienne sur l'épigraphie du monde romain organisée par l'Université de Roma - "La Sapienza” et l'École Française de Rome, Rome, 5-6 juin 1992 (Coll. de l'École Fr. de Rome 193), Paris - Rome 1994.

Epigrafia d'età angustea = G. PACI (Ed.), Contributi all'epigrafia d'età angustea. Actes de la XIII Rencontre franco-italienne sur l'épigraphie du monde romain, Macerata, 9-11 settembre 2005 (Ichnia 8), Macerata - Tivoli (RM) 2007.

Epigrafia di confine $=$ M. G. ANGELI BerTINELLI - A. DONATI (Ed.), Epigrafia di confine, confine dell'epigrafia. Atti del Colloquio AIEGL - Borghesi 2003, Bertinoro, 10-12 ottobre 2003 (Epigrafia e Antichità 21), Bologna - Faenza 2004.

Epigrafia e archeologia romana = G. PACI (Ed.), Epigrafia e archeologia romana nel territorio marchigiano. In memoria di Lidio Gasperini. Atti del Convegno, Macerata, 22-23 aprile 2013 (Ichnia 13), Macerata - Tivoli (RM) 2013.

Epigrafia e ordine senatorio I-II = Epigrafia e ordine senatorio. Atti del colloquio internazionale AIEGL, Roma, 14-20 maggio 1981 I-II (Tituli 4-5), Roma 1982 [1984].

Epigrafia e ordine senatorio, 30 anni dopo $\mathrm{I}-\mathrm{II}=\mathrm{M}$. L. CALDELLI - G. L. GREGORI (Ed.), Epigrafia e ordine senatorio, 30 anni dopo. Atti della XIX ${ }^{e}$ Rencontre franco-italienne sur l'épigraphie du monde romain, Roma, 21-23 marzo 2013 I-II (Tituli 10), Roma 2014.

Epigrafia e politica $=$ S. SEgENNI - M. Bellomo (Ed.), Epigrafia e politica. Il contributo della documentazione epigrafica allo studio delle dinamiche politiche del mondo romano (Milano, 28-29 gennaio 2015) (Consonanze 4), Milano 2017.

Epigrafia e territorio II = M. PANI (Ed.), Epigrafia e territorio Politica e società. Temi di antichità romane II (Documenti e studi 5), Bari 1987.

Epigrafia e territorio III = M. PANI (Ed.), Epigrafia e territorio. Politica e società. Temi di antichità romane III (Documenti e studi 17), Bari 1994.

Epigrafia e territorio IV = M. PANI (Ed.), Epigrafia e territorio. Politica e società. Temi di antichità romane IV (Documenti e studi 19), Bari 1996.

Epigrafia e territorio VII = M. PANI (Ed.), Epigrafia e territorio. Politica e società. Temi di antichità romane VII (Documenti e studi 37), Bari 2004.

Epigrafia e territorio VIII = M. PANI (Ed.), Epigrafia e territorio. Politica e società. Temi di antichità romane VIII (Documenti e studi 42), Bari 2007. 
Epigrafia e territorio IX = M. CHelotti - M. SILvestrinI (Ed.), Epigrafia e territorio. Politica e società. Temi di antichità romane IX (Documenti e studi 54), Bari 2013.

Epigrafia e territorio $\mathrm{X}=\mathrm{M}$. Chelotti $-\mathrm{M}$. Silvestrin (Ed.), Epigrafia e territorio. Politica e società. Temi di antichità romane X (Documenti e studi 64), Bari 2016.

Epigrafía Jurídica $=$ C. CASTILlo et AL. (Ed.), Novedades de Epigrafía Jurídica Romana en el ultimo decenio. Actas del Coloquio Internacional A.I.E.G.L., Pamplona, 9-11 de abril de 1987, Pamplona 1989.

Epigrafia romana in area adriatica $=$ G. PACI (Ed.), Epigrafia romana in area adriatica. Actes de la IX $X^{e}$ Rencontre francoitalienne sur l'épigraphie du monde romain, Macerata, 1011 novembre 1995 (Ichnia 2), Macerata - Pisa 1998.

Equi = S. LAPENNA (Ed.), Gli Equi tra Abruzzo e Lazio. Catalogo della mostra, Sulmona (AQ) 2004.

Equicoli= G. Alvino (Ed.), Gli Equicoli. I guerrieri delle montagne, Roma 2004

Erkelenz, Optimo praesidi $=$ D. Erkelenz, Optimo praesidi. Untersuchungen zu den Ehrenmonumenten für Amtsträger der römischen Provinzen in Republik und Kaiserzeit (Antiquitas 1. 52), Bonn 2003.

Ernout, Notes $=$ A. Ernout, Notes de philologie latine (Centre de Recherches d'Histoire et de Philologie de la IVe Section de l'École pratique des Hautes Études III. Hautes Études du Monde Gréco-Romaine 3), Genève - Paris 1971.

Ernout, Recueil ${ }^{4}=$ A. Ernout, Recueil de textes latins archaïques $^{4}$, Paris 1973.

ErTman, Curatores viarum $=$ PH. C. ERTMAn, Curatores viarum: a Study of the Superintendents of Highways in ancient Rome, Ann Arbor (MI) 1976.

Esch, Römische Straßen = A. Esch, Römische Straßen in ibrer Landschaft. Das Nachleben antiker Straßen um Rom mit Hinweisen zur Begehung im Gelände, Mainz 1997.

Esclaves = M. Dondin-PAYre - N. Tran (Ed.), Esclaves et maîtres dans le monde romain. Expressions épigraphiques de leurs relations (Coll. de l'École Fr. de Rome 527), Rome 2017.

Espluga, Silloge di Faenza $=$ X. Espluga, Silloge di Faenza e la tradizione epigrafica di Verona (Epigrafia e Antichità 39), Bologna 2017.

Est enim ille flos Italiae $=\mathrm{P}$. Basso $-\mathrm{A}$. BuONOpane $-\mathrm{A}$. Cavarzere - S. Pesavento Mattioli (Ed.), Est enim ille flos Italiae. Vita economica e sociale nella Cisalpina romana. Atti delle giornate di studi in onore di Ezio Buchi, Verona, 30 novembre - 1 dicembre 2000, Verona 2008.

Estudios = B. García-HernÁNDEZ - R. López Gregoris - E. Nieto Ballester - M. E. Torrego (Ed.), Estudios de lingüistica Latina. Actas del IX Coloquio Internacional de Lingüística Latina. Universidad Autonóma de Madrid, 14 18 de abril de 1997, Madrid 1998.

Estudios Marc Mayer = G. Baratta - A. Buonopane - J. Velaza (Ed.), Cultura epigráfica y cultura literaria. Estudio en Homenaje a Marc Mayer i Olivé (Epigrafia e Antichità 44), Bologna - Faenza 2019.

Euler, Donom do = W. Euler, Donom do. Eine figura etymologica der Sprachen Altitaliens, Innsbruck 1982.

Evangelisti, Epigrafia anfiteatrale $=$ S. Evangelisti, Epigrafia anfiteatrale dell'Occidente romano VIII. Regio Italiae I, 2: Campania praeter Pompeios (Vetera 18), Roma 2011.

Evans, Cults = E. C. Evans, The Cults of the Sabine Territory (Pap. Mon. Am. Acad. Rome 11), Roma 1939.

Evergetismo = F. Cancrini - Chr. Delplace - S. M. Marengo, L'evergetismo nella regio $V$ (Picenum) (Picus. Supplementi 8), Tivoli 2001.

Ex adversis fortior resurgo $=\mathrm{R}$. BERTINI CONIDI - F. LONGO (Ed.), Ex adversis fortior resurgo. Miscellanea in ricordo di Patrizia Sabbatini Tumolesi (Arti Spazi Scritture. Storia antica 7), Ospedaletto (PI) 2008
Ex officina $=$ C. Fernández Martínez - M. Limón BeléN J. Gómez Pallarès - J. del Hoyo Calleja (Ed.), Ex officina. Literatura epigráfica en verso (Linguistica 128), Sevilla 2013.

FABBI, Antichità = A. FABBI, Antichità umbre (natura, storia, arte), Spoleto (PG) 1971.

FАввІ, Guida = A. FАввI, Guida alla Valnerina, Abeto $(\mathrm{PG})$ 1977.

Fabbi, Preci $=$ A. Fabbi, Preci e la Valle Castoriana. Terra ignorata, Spoleto (PG) 1963.

FabBI, Storia dei Comuni I = A. FabBi, Storia dei Comuni della Valnerina I, Abeto (PG) 1976.

Fabbi, Storia e arte $=$ A. Fabbi, Storia e arte nel comune di Cascia, Cascia (PG) 1975.

Fabbi, Studio = A. Fabbi, Studio storico-artistico su Aliena, Spoleto (PG) 1973.

Fавві, Visso = A. Faвbi, Visso e le sue valli, Spoleto (PG) 1965 (ed. altera Spoleto 1977).

FABBRI, Mater florum = L. FABBRI, Mater florum. Flora e suo culto a Roma (Bibliotheca dell'"Archivum Romanicum" I.492), Firenze 2019.

FABRE, Libertus = G. FABRE, Libertus. Recherches sur les rapports patron-affranchi à la fin de la République romaine (Coll. de l'École Fr. de Rome 50), Rome 1981.

Fagan, Bathing = G. G. Fagan, Bathing in Public in the Roman World, Ann Arbor (MI) 2002

Falacrinae = F. CoArelli - R. CAscino - V. Gasparini (Ed.), Falacrinae. Le origini di Vespasiano. Catalogo della mostra (Cittareale [RI], Museo Civico - Auditorium di S. Maria, 18 luglio 2009 - 19 gennaio 2010), Roma 2009.

Fana III = S. CAPINI - P. CURCI - M. R. Picuti (Ed.), Fana, templa, delubra. Corpus dei luoghi di culto dell'Italia antica III. Regio IV: Alife, Bojano, Sepino, Roma 2015.

FANT, Curatores rei publicae $=$ J. C. FANT, The curatores re publicae in Italy from their Inception to the Death of Commodus (Xerox Univers. Microfilms), Ann Arbor (MI) 1976.

Fanum Voltumnae = G. M. Della Fina (Ed.), Il fanum Voltumnae e $i$ santuari comunitari dell'Italia antica. Atti del XIX Convegno Internazionale di Studi sulla Storia e l'Archeologia dell'Etruria (Ann. "Claudio Faina” 19), Orvieto (TR) - Roma 2012.

FaOro, Praefectus = D. FaOro, Praefectus, procurator, praeses. Genesi delle cariche presidiali equestri nell'Alto Impero Romano (Studi Udinesi sul Mondo Antico 8), Firenze Città di Castello (PG) 2011.

Farfa = R. Dondarini (Ed.), Farfa, abbazia imperiale. Atti del convegno internazionale, Farfa - Santa Vittoria in Matenano, 25-29 agosto 2003, S. Pietro in Cariano (VR) 2006.

Farina - Fiorillo - Del SANTO - Vitelli, Alife romana $=\mathrm{S}$. Farina - G. Fiorillo - A. Del Santo - S. Vitelli, Alife romana. Contributo alla conoscenza delle opere pubbliche e private, Napoli 1982

FAsolini, Aggiornamento $=$ D. FAsolinI, Aggiornamento bibliografico ed epigrafico ragionato sull'imperatore Claudio, Milano 2006.

FasolinI, Tribù romane = D. FAsolinI, Le tribù romane della Hispania Tarraconensis. L'ascrizione tribale dei cittadini romani nelle testimonianze epigrafiche, Milano 2012.

Fasolo, Paesaggi $=$ M. Fasolo, Antichi paesaggi agrari d'Italia nelle banche dati dell'AGEA, Roma 2006.

FAure, Centurions $=$ P. FAURE, L'aigle et le cep. Les centurions légionnaires dans l'Empire des Sévères (Scripta antiqua 54), Bordeaux 2013.

Fausti, Chiesa = L. Fausti, La Chiesa Priorale di San Felice di Narco, Spoleto (PG) 1922

FAyer, Dea Roma = C. Fayer, Il culto della dea Roma. Origine e diffusione nell'impero, Pescara 1976. 
Febonio = V. Esposito - G. Morelli (Ed.), Muzio Febonio nel Quarto Centenario della nascita (1597-1997). Atti del Convegno di studi, Avezzano, 9-10 maggio 1998, L’Aquila 2000.

Federazioni $=$ L. Aigner Foresti - A. Barzanò - C. Bearzot - L. Prandi - G. Zecchini (Ed.), Federazioni e federalismo nell'Europa antica. Alle radici della casa comune europea. Bergamo, 21-25 settembre 1992 I-II, Milano 1994.

Felletti Maj, Tradizione italica $=$ B. M. Felletti MaJ, La tradizione italica nell'arte romana (Stud. Arch. 3), Roma 1977.

Fernández Martínez, Poesía epigráfica $=$ C. Fernández Martínez, Poesía epigráfica latina (Biblioteca Clásica Gredos 259), Madrid 1998.

Fernique, Inscriptions inédites = E. Fernique, Inscriptions inédites du pays des Marses (Bibl. des Écoles Fr. d'Athènes et de Rome 5), Paris 1879.

Ferone, Opuscula I-III = C. Ferone, Opuscula, a cura di A. Russi I-III (Gervasiana. Opuscula 1), San Severo (FG) 2013.

Ferrara, Canneto = V. Ferrara, Canneto sul Trigno, Lanciano - Vasto $(\mathrm{CH}) 1989$.

Ferrara, Cripta $=$ V. Ferrara, La cripta e il battistero paleocristiano di Trivento, Penne (AQ) 1991.

Ferrara, Diocesi $=$ V. Ferrara, Diocesi di Trivento I. Periodo delle origini, Roma 1990

Festschrift Vittinghoff = W. ECK - H. GALSTERER - H. WOLFF (Ed.), Studien zur antiken Sozialgeschichte. Festschrift F. Vittinghoff, Köln - Wien 1980.

Festschrift Weber = F. BEUTLER - W. HAMETER (Ed.) "Eine ganz normale Inschrift” ... Und ähnliches zum Geburtstag von Ekkehard Weber. Festschrift zum 30. April 2005 (Althistorisch-Epigraphische Studien 5), Wien 2005.

Fides amicorum = G. FIRPO (Ed.), Fides amicorum. Studi in onore di Carla Fayer, Pescara 2011.

Filo di Arianna = R. Tuteri (Ed.), Un filo di Arianna. Percorsi del Museo Archeologico di Sulmona tra pannelli e schede, Atri (TE) 2011

Filomusi-Guelfi, Iscrizione latina $=$ F. Filomusi-Guelfi, Sopra un'iscrizione latina scoperta in Tocco a Causaria, Chieti 1881.

Filomusi-Guelfi, Strenna $=$ L. Filomusi-Guelfi, Strenna $d i$ Tocco a Casauria, Chieti 1881.

Finanze municipali $=I l$ capitolo delle entrate nelle finanze municipali in Occidente ed in Oriente. Actes de la X $X^{e}$ Rencontre franco-italienne sur l'épigraphie du monde romain, Rome, 27-29 mai 1996 (Coll. de l'École Fr. de Rome 256), Roma 1999.

Finelli, Città di Alife = F. S. Finelli, Città di Alife e diocesi. Cenni storici, Scafati (SA) 1928.

Finocchietti, Molise = L. Finocchietti, Il Molise in età tardo-antica. Città e campagne tra il III e il VI secolo d. C. (BAR. International Series 2337), Oxford 2012.

A. Fiocca, Aufidena = A. Fiocca, Aufidena, oggi Castel di Sangro. Edizione corretta e accresciuta, Roma 1899

L. Fiocca, Castel di Sangro = L. Fiocca, Castel di Sangro ed $i$ suoi monumenti, Palermo 1903.

Fiocchi Nicolai, Cimiteri $=$ V. Fiocchi Nicolai, I cimiter paleocristiani del Lazio II. Sabina (Mon. Ant. Crist. $2^{\circ}$ ser., 20), Città del Vaticano 2009.

Fiocchi Nicolai - Ricciardi, Catacomba di S. Vittoria = V. FiocChi Nicolai - M. Ricciardi, La catacomba di S. Vittoria a Monteleone Sabino (Trebula Mutuesca). Con appendice di B. MAZZEI (Scavi e restauri pubblicati a cura della Commissione di archeologia sacra 2), Città del Vaticano 2003.

FIORANI, Una città romana $=$ G. FIORANI, Una città romana: Magliano dei Marsi (dalle origine al Medioevo), Roma 1978.
Fiordigigli, San Giustino = G. Fiordigigli, San Giustino. Vescovo di Chieti - Confessore nei Vestini, S. Gabriele (TE) 1999.

Fiorelli, Catalogo = G. Fiorelli, Catalogo del Museo Nazionale di Napoli. Raccolta epigrafica II. Iscrizioni latine, Napoli 1868.

FIRA ${ }^{2}$ I-III = S. RICcobono et AL. (Ed.), Fontes iuris Romani antejustiniani $^{2}$ I-III, Florentiae 1940/43 (partis III editio altera appendice aucta, Florentiae 1969).

Firpo, Roma = G. Firpo, Roma e i veteres hostes (Saggi di storia antica 38), Roma 2015.

FISHWICK, Imperial Cult I-II = D. FisHwICK, The Imperial Cult in the Latin West. Studies in the Ruler Cult of the Western Provinces of the Roman Empire I-II (EPRO 108), LeidenNew York - Köln 1987/92.

FITZ, Laufbahn der Statthalter = J. FITZ, Die Laufbahn der Statthalter in der römischen Provinz Moesia inferior, Weimar 1966.

Fondation = S. Agusta-Boularot - S. Huber - W. VaN ANDringa (Ed.), Quand naissent les dieux. Fondation des sanctuaires antiques: motivations, agents, lieux (Coll. de l'École Fr. de Rome 534), Roma 2017.

Fonti latine e greche I-II = M. BuONOCORE - G. Firpo, Fonti latine e greche per la storia dell'Abruzzo antico I-II (DASP. Documenti per la Storia d'Abruzzo 10. 1-2), Padova 1991 - L'Aquila 1998.

ForA, Epigrafia anfiteatrale $=$ M. ForA, Epigrafia anfiteatrale dell'Occidente romano IV. Regio Italiae I: Latium (Vetera 11), Roma 1996.

FORA, Munera gladiatoria $=$ M. FORA, I munera gladiatoria in Italia. Considerazioni sulla loro documentazione epigrafica (Istituto adriatico per la storia del Principato fra Adriano e Diocleziano 1), Napoli 1996.

Forbis, Municipal Virtues = E. Forbis, Municipal Virtues in the Roman Empire. The Evidence of Italian Honorary Inscriptions (Beiträge zur Altertumskunde 79), Stuttgart Leipzig 1996.

Forma = Forma . La città antica e il suo avvenire, Roma 1985. Forme e strutture $=$ A. ANCILLOTTI - A. CALDERINI - R. MASSARELLI (Ed.), Forme e strutture della religione nell'Italia mediana antica. Forms and Structures of Religion in Ancient Central Italy. III Convegno Internazionale dell'Istituto di Ricerche e Documentazione sugli antichi Umbri, Perugia - Gubbio, 21-25 settembre 2011 (Stud. Arch. 215), Roma 2016.

Forme municipali = S. EvANGELISTI - C. RICCI (Ed.), Le forme municipali in Italia e nelle province occidentali tra i secoli I a. C. e III d. C. Atti della "XXI Rencontre franco-italienne sur l'épigraphie du monde romain", Campobasso, 24-26 settembre 2015 (Insulae Diomedeae 29), Bari 2017.

ForNARI, $m s .=$ F. ForNARI, Breviarium manu scriptum d. $9 \mathrm{~m}$. Sept. a. 1916 servatum Romae apud Archivio Centrale dello Stato (EUR). MPI. Dir. Gen. AABBAA. Antichità e Scavi. Divisione I, 1916/19, b. 641, fasc. "Prata d'Ansidonia".

ForNI, Esercito = G. ForNI, Esercito e marina di Roma antica (Mavors 5), Amsterdam 1992.

FORNI, Reclutamento $=$ G. FORNI, Il reclutamento delle legioni da Augusto a Diocleziano, Milano - Roma 1953.

ForNI, Scripta minora = G. ForNI, Le tribù romane IV. Scripta minora (Historica 6), Roma 2006.

FornI, Tribù = G. ForNI, Le tribù romane I. Tribules 1-4 (Historica 3. 5. 7. 10), Roma 1996/2012.

Fracassi, San Pelino = P. Fracassi, San Pelino la capitale de $i$ Marsi Anxantini, L'Aquila 1988.

Fraccaro, Opuscula III = P. Fraccaro, Opuscula III. Scritti di Topografia e di Epigraphia. Vol. I, Testo. Vol. II, Tavole, Pavia 1957. 
France, Quadragesima Galliarum = J. France, Quadragesima Galliarum. L'organisation donanière des provinces alpestres, gauloises et germaniques de l'Empire romain $\left(I^{e r}\right.$ siècle avant J.-C. - III siècle après J-C.) (Coll. de l'École Frecle avant Je Rome 278), Paris - Rome 2001.

Francesco Saverio Cremonese $=$ M. SAlzano (Ed.), Il quarantennale (30 settembre 1977 - 30 settembre 2017) ed il contributo di Francesco Saverio Cremonese all'epigrafia e all'archeologia del Molise, Agnone (IS) 2018.

Franceschini, Memorie storiche $=$ M. Franceschini, Memorie storiche di Cascia, Cascia 1913 (= Documentazione Ritiana Antica III, Cascia [PG] 1968, 63-121).

Franceschini, $m s$. I = M. FranCESChini, Raccolta delle lapid esistenti in Cascia e nel suo territorio (liber manu scriptus a. 1810, servatus Cascia in aedibus Franceschini sine inv. n.; exemplum lucis ope impressum exstat Cascia in bibliotheca municipali).

Franceschini, $m s$. II = M. FranCESChini, Ristretto istorico d Cascia e del suo territorio (liber manu scriptus a. 1810, servatus Cascia in aedibus Franceschini sine inv. n.; exemplum lucis ope impressum exstat Cascia in bibliotheca municipali).

Frank, Economic Survey I-VI = T. Frank, An Economic Survey of Ancient Rome I-V, Baltimore 1933/40, VI [Index], Paterson (NJ) 1959.

Franzoni, Habitus = C. Franzoni, Habitus atque habitudo militis: monumenti funerari di militari nella Cisalpina Romana (Stud. Arch. 45), Roma 1987.

Fraschetti, Roma e il principe $e^{2}=$ A. Fraschetti, Roma e il principe $^{2}$ (Biblioteca Universale Laterza 571), Roma - Bari 2005.

Fratianni, Terventum $=$ G. Fratianni, Terventum. Carta Archeologica della media valle del Trigno (Riv. Top. Ant. Supplemento 6), Galatina (LE) 2010.

Freis, Cohortes urbanae $=\mathrm{H}$. FreIs, Die cohortes urbanae (Epigr. Studien 2), Köln - Graz 1967.

Frenz, Römische Grabreliefs = H. G. Frenz, Römische Grabreliefs in Mittel- und Süditalien (Archaeologica 37), Roma 1985.

FrIEDL, Konkubinat $=$ R. FrIedL, Der Konkubinat im kaiser zeitlichen Rom. Von Augustus bis Septimius Severus (Historia Einzelschriften 98), Stuttgart 1996.

FrIedläNDER, Darstellungen ${ }^{9}=$ L. FrIEDLÄNDER, Darstellungen aus der Sittengeschichte Roms ${ }^{9}$, Leipzig 1919/20.

FuCINESE, Eremo = D. V. FuCINESE, L'eremo di S. Venanzio in Raiano, Raiano (AQ) 1976.

FuCINESE, Raiano = D. V. FuCINESE, Raiano. Notizie storiche e vita tradizionale, L'Aquila 1971.

FuCINESE, Raiano $e$ dintorni $=$ D. V. FuCINESE, Raiano $e$ dintorni. L'immagine e la storia, Raiano (AQ) 1987.

Fucinese, Sant'Angelo di Vittorito = D. V. Fucinese, Sant'Angelo di Vittorito e la presenza del 'Maestro del trittico' di Beffi nella Valle Peligna, Raiano (AQ) 2003.

Fucino I = U. Irti - G. Grossi - V. Pagani (Ed.), Il Fucino e le aree limitrofe nell'antichità. Atti del Convegno di Archeologia, Avezzano, 10-11 novembre 1989, Roma 1991.

Fucino II = E. Burri - A. Campanelli (Ed.), Sulle rive della memoria. Il lago Fucino e il suo Emissario, Pescara 1994.

Fucino III = Il Fucino e le aree limitrofe nell'antichità. Atti del II Convegno di Archeologia in ricordo di Antonio Mario Radmilli e Giuliano Cremonesi, Museo di Preistoria, Celano - Paludi, 26-28 novembre 1999, Avezzano (AQ) 2001.

Fucino IV = D. Liberatore - M. J. Strazzulla, Fucino. Studi sulla cultura figurativa (Insulae Diomedeae 6), Bari 2007.

Fucino V = G. Grossi - U. Irti - C. Malandra (Ed.), Il Fucino e le aree limitrofe nell'antichità. Atti del III Convegno di Archeologia in ricordo di Walter Cianciusi, Castello
Orsini, Avezzano, 13-15 novembre 2009, Avezzano (AQ) 2011.

Fucino VI = Il Fucino e le aree limitrofe nell'antichità. Archeologia e rinascita culturale dopo il sisma del 2015. Atti del IV Convegno di Archeologia, Castello Orsini, Avezzano, 20-23 maggio 2015, Avezzano (AQ) 2016.

Fucino cento anni $=$ Fucino cento anni 1877-1977. Atti degli incontri e dei convegni svoltisi per il Centenario del prosciugamento del Fucino e per il Venticinquennale della Riforma Agraria, 14 maggio 1977 - 4 marzo 1978, Avezzano (AQ) 1979 (editio tertia Teramo 1993).

Fusco, Gestione delle acque = A. Fusco, La gestione delle acque nelle civitates dell'Italia romana. La documentazione epigrafica (liber machina dactylographica redactus thesis doctoris dignitatis nominisque a. 2011/13 Palermo impetratae apud Università degli Studi. Dipartimento di Beni Culturali - Studi Culturali).

GABBA, Italia romana $=$ E. GABBA, Italia romana (Biblioteca $d i$ Athenaeum 25), Como 1984.

Gaetano Marini = M. Buonocore (Ed.), Gaetano Marini (1742-1815) protagonista della cultura europea. Scritti per il bicentenario della morte I-II (BAV. Studi e testi 492493), Città del Vaticano 2015.

GAGgiotti, Fontana = M. GAGgiotTi, La fontana del grifo a Saepinum (Documenti 3), Roma 1973.

GaLIANI, $m s .=$ F. GaLIANI, Libri manu circ. saec. XVIII medio scripti sign. XXX.C.12 et XXXI.A.10 nunc servati Neapoli in bibliotheca societatis Società Napoletana di Storia Patria, de quibus a. 2017 in. me certiorem fecit Umberto SOLDOVIERI.

Galletier, Étude = É. Galletier, Étude sur la poésie funéraire romaine d'après les inscriptions, Paris 1922.

Galli, Ricerche $=$ L. Galli, Ricerche sui collegi romani (liber machina dactylographica redactus thesis doctoris dignitatis nominisque a. 1997/98 Romae impetratae apud Università degli Studi di Roma "La Sapienza", Scuola Nazionale di Archeologia).

A. Gallo, Amiternum = A. Gallo, Amiternum, L'Aquila 1904 (lucis ope iterum impressum Avezzano (AQ) [1985?]).

Gallo, Prefetti $=$ Annarosa Gallo, Prefetti del pretore e prefetture. L'organizzazione dell'agro romano in Italia (IV-I sec. a. C.) (Documenti e studi 68), Bari 2018.

Galluppi, Faifoli $=$ M. Galluppi, Faifoli, città del Sannio durante l'epoca repubblicana ed imperiale di Roma. Documenti. Epigrafi. Conclusioni storiche, Roma 1927.

Galluppi, S. Maria di Canneto = M. Galluppi, S. Maria di Canneto sul Trigno nell'archeologia, nella storia e nell'arte, Roma 1941.

Galsterer, Herrschaft $=$ H. Galsteren, Herrschaft und Verwaltung im republikanischen Italien. Die Beziehungen Roms zu den italischen Gemeinden vom Latinerfrieden 338 v. Chr. bis zum Bundesgenossenkrieg 91 v. Chr. (MBPAR 68), München 1976.

GARAMPI, $m s$. = Adversaria itinerarii a IOSEPHO GARAMPI a. 1748 facti manu scripta servata in codice Archivi Secreti Apostolici Vaticani sign. Fondo Garampi 133 f. 282-284. 287-306 (gratias ago collegae GiovanNi VenditTi, qui me certiorem fecit; vide EUND., BDASP 108, 2017 [2018], 129-165).

García Valdecasas, La fórmula $=$ A. García Valdecasas, $\mathrm{La}$ fórmula $\mathrm{H}-\mathrm{M}-\mathrm{H}-\mathrm{HH}-\mathrm{S}$ en las fuentes epigráficas romanas. Contribución a la historia de los sepulcros familiares y hereditarios en el derecho romano (Anuario de historia del derecho español 5), Madrid 1929.

GARRUCCI, Isernia $=$ R. GARRUCCI, La storia di Isernia raccolta dagli antichi monumenti, Napoli 1848. 
Garrucci, Sylloge = R. GARrucci, Sylloge Inscriptionum Latinarum aevi Romanae rei publicae usque ad C. Iulium Caesarem plenissima I-II, Augustae Taurinorum 1875/77.

Garrucci a Benevento = C. Ferone - I. M. IAsiello, Garrucci a Benevento. Temi e modi di uno scontro intellettuale alle origini della riscoperta archeologica di Benevento (Collezione storica 4), Roma 2008.

Garton, Roman Theatre $=$ Ch. Garton, Personal Aspects of the Roman Theatre, Toronto 1972.

Gasdia, Campobasso = V. E. Gasdia, Storia di Campobasso, [s. 1.] 1960.

GaSperini Spoletium - Nursia $=$ M. GASPerini, Da Spoletium a Nursia. Viabilità, topografia e dinamiche insediative (liber machina dactylographica redactus thesis doctoris dignitatis nominisque a. 2012/13 Romae impetratae apud Università degli Studi di Roma "La Sapienza") [http:// hdl.handle.net/10805/2314].

Gattinara, Tagliacozzo = G. Gattinara, Storia di Tagliacozzo dalla origine ai giorni nostri con brevi cenni sulla regione marsicana, Città di Castello (PG) 1894.

Geiger, Sacerdotibus = F. GeIger, De Sacerdotibus Augustorum Municipalibus (Dissertationes Philologicae Halenses 23), Halis Saxonum 1913.

Genres épigraphiques = E. Dupraz - W. Sowa (Ed.), Genres épigraphiques et langues d'attestation fragmentaire dans l'espace méditerranéen (Cahiers de l'ERIAC 9), MontSaint-Aignan (Rouen) 2015.

Gentes et Principes = M. R. SANZI Di Mino - L. Nista (Ed.), Gentes et Principes. Iconografia romana in Abruzzo, Pisa 1993.

Germanico $=$ G. Bonamente - M. P. Segoloni (Ed.), Germa nico: la persona, la personalità, il personaggio. Atti del Convegno, Macerata - Perugia, 9-11 maggio 1986 (Università degli Studi di Macerata. Atti di Convegni 4), Roma 1987.

Gesellschaft und Recht $=$ M. N. ANDREEv et AL. (Ed.), Gesellschaft und Recht im Griechisch-Römischen Altertum I-II (Deutsche Akademie der Wissenschaften zu Berlin. Schriften der Sektion für Altertumswissenschaft 52), Berlin 1968/ 69.

Giannangeli, Barisciano = R. Giannangeli, Terra di Barisciano. Ricerca storica, L'Aquila 1974.

Ginestet, Organisations de la jeunesse $=$ P. Ginestet, Les organisations de la jeunesse dans l'Occident Romain (Coll. Latomus 213), Bruxelles 1991.

Giocondo, Borg. lat. = Giovanni Giocondo dA VeronA, Collectio inscriptionum Latinarum et Graecarum (liber temporis spatio inter a. 1490 et a. 1498 comprehenso ab Iacobo Aurelio Questenberg exaratus olim apud Sacram Congregationem de Propaganda Fide nunc in Bibliotheca Apostolica Vaticana adservatus sign. Borg. lat. 336).

Giornata Amaduzziana = Atti della Terza Giornata Amaduzziana. Rubiconia Accademia dei Filopatridi, Savignano sul Rubicone, 30 marzo 2003 (Rubiconia Accademia dei Filopatridi. Centro di Studi Amaduzziani 4), Viserba (RN) 2004.

Giornata di studi $=$ S. ANTOLINI - A. ARNALDi - E. LANZILLOTTA (Ed.), Giornata di studi per Lidio Gasperini. Roma, 5 giugno 2008 (Themata 4), Tivoli (RM) 2010.

GIRARD, Textes de droit romain ${ }^{6}=$ P. F. GIRARD, Textes de droit romain. 6 éd. entièrement revue et augmentée par $\mathrm{S}$. SEnN, Paris 1937 (= Les lois des Romains. 7 éd. par un groupe de romanistes des "Textes de droit romain", Tome II de P. F. Girard et F. Senn, pubblicazione curata da V. GIUfFrè̀, Napoli 1977 [Pubblicazioni della Facoltà di Giurisprudenza dell'Università di Camerino 12]).
GiUfFrè, Documenti testamentari $=$ V. GiUfFrè, Document $i$ testamentari romani (Testi per esercitazioni 3), Milano 1974.

Giustiniani, Dizionario $=$ L. Giustiniani, Dizionario Geografico Ragionato del Regno di Napoli, Napoli 1816.

Giustizia, Territorio = F. Giustizia, Paletnologia ed archeologia di un territorio, Roma 1985.

GofFin, Euergetismus = B. Goffin, Euergetismus in Oberitalien (Habelts Dissertationsdrucke, Reihe Alte Geschichte 46), Bonn 2002

GolinI, Vittorito = D. GolinI, Vittorito nell'antichità. Archeologia di un insediamento, Roma 1994.

Golvers, Studie = N. Golvers, Studie over het toponymisch materiaal van de antieke Sabina, Diss. Leuven 1989 (non vidi).

Gordon, Album I-III = A. E. Gordon - J.S. Gordon, Album of Dated Latin Inscriptions. Rome and the Neighborhood I-III. Text and Plates. IV. Indexes, Berkeley - Los Angeles 1958/65.

GoRDON, Introduction = A. E. GoRDon, Illustrated Introduction to Latin Epigraphy, Berkeley - Los Angeles - London 1983.

Gori, Nuova guida $=$ F. GorI, Nuova guida storica, artistica, geologica ed antiquaria da Roma a Tivoli e Subiaco alla grotta di Collepardo alle valli dell'Amsancto e al lago Fucino V, Roma 1864

GorI, Relazione $=$ F. GorI, Relazione delle ultime scoperte di antichità nella regione sabina, Rieti 1900.

Granarolo, D'Ennius à Catulle = J. Granarolo, D'Ennius à Catulle: recherches sur les antécédents romains de la "poésie nouvelle", Paris 1971.

Granino Cecere, Flaminato femminile = M. G. Granino Cecere, Il flaminato femminile imperiale nell'Italia romana (Urbana species 2), Roma 2014.

Greco, Città di Penne = C. Greco, Città di Penne, Penne (AQ) 1999.

Greco, Civitaquana = C. Greco, Civitaquana, storia di un'abazia e di due castelli, Penne (AQ) 2002.

Greco, Statuti $=$ C. Greco, Statuti della città di Penne (sec. XV) ovvero Codice Catena, Penne (AQ) 2003.

Gregori, Brescia romana I-II = G. L. Gregori, Brescia romana. Ricerche di prosopografia e storia sociale I. I documenti. II. Analisi dei documenti (Vetera 7. 13), Roma 1990/2000.

Gregori, Epigrafia anfiteatrale = G. L. Gregori, Epigrafia anfiteatrale dell'Occidente romano II. Regiones Italiae VIXI (Vetera 4), Roma 1989.

GREGORI, Ludi e munera = G. L. GREGORI, Ludi e munera. 25 anni di ricerche sugli spettacoli d'età romana. Scritti vari rielaborati e aggiornati con la collaborazione di $\mathrm{G}$. CRIMI $e$ M. Giovagnoli (Scienze sociali. Studi e ricerche 13), Milano 2011.

Grelle, Canosa romana = F. Grelle, Canosa romana (Saggi di storia antica 5), Roma 1993.

Grossi, Angitia = G. Grossi, La città di Angitia, il lucus Angitiae e le origini di Luco dei Marsi, Avezzano (AQ) 1981.

Grossi, Avezzano = G. Grossi, Le origini di Avezzano, Avezzano (AQ) 2000.

Grossi, Celano = G. Grossi, Celano. Storia. Arte. Archeologia, Celano (AQ) 1998

Grossi, Corcumello = G. Grossi, Corcumello dall'antichità al medioevo, Corcumello (AQ) 1994.

Grossi, Insediamenti = G. Grossi, Insediamenti italici nel Cicolano: territorio della "res publica Aequiculanorum" (Monografia e supplemento della $1^{\circ}$ Conferenza di aggiornamento scolastico su temi storico-territoriali del Cicolano), L'Aquila 1984.

Grossi, Marsica = G. Grossi, Marsica. Guida storica-archeologica, Avezzano (AQ) 2002 
Grossi, Marsica Sacra = G. Grossi, Marsica Sacra. Chiese, Celle e Monasteri (IV-XII secolo), Avezzano (AQ) 2004.

Grossi, Ricerca = G. Grossi, Alla ricerca di Marsi ed Equi (XVI-XXI secolo), Avezzano (AQ) 2019.

Grossi, Trasacco = G. Grossi, Guida turistica di Trasacco, Trasacco (AQ) 1996

Grossi - D'Amore, Capistrello = G. Grossi - F. D'Amore, Capistrello. Storia. Arte. Archeologia, Isola Liri (FR) 2000.

Grossi Gondi, Trattato $=$ F. Grossi Gondi, Trattato di epigrafia cristiana latina e greca del mondo romano occidentale, Roma 1920 (lucis ope iterum impressum [I monumenti cristiani dei primi secoli 1], Roma 1968).

Gualtieri, Inscriptiones = F. S. Gualtieri, Inscriptiones repertae in ruderibus, et agro Antini, nunc Civita d'Antino (duo folia manu scripta (a-b) adplicita in fine voluminis Historia Marsorum Mutii Phoebonii nunc servati in Bibliotheca Apostolica Vaticana sign. Racc. Gen. Storia IV. 2231).

GuARDABASSI, Indice $=$ M. Guardabassi, Indice - Guida de monumenti pagani e cristiani riguardanti l'istoria e l'arte esistenti nella provincia dell'Umbria, Perugia 1872 (editio altera Bologna 1968).

GuARDABASSI, Lettere $=$ M. Guardabassi, Lettere (libri manu scripti post saec. XIX medium exarati servati Perugia in Biblioteca comunale Augusta sub Fondo Guardabassi).

Guardabassi, Taccuini = M. Guardabassi, Taccuini (libri manu scripti post saec. XIX medium exarati servati Perugia in Biblioteca comunale Augusta sub Fondo Guardabassi).

GuARducci, Scritti scelti = M. GuARduccI, Scritti scelti sulla religione greca e romana nel Cristianesimo (EPRO 98), Leiden 1983.

Guattani, Monumenti sabini = G. A. Guattani, Monumenti sabini I-III, Roma 1827/30.

Guérin-Beauvois, Thermalisme = M. Guérin-Beauvois, Le Thermalisme romain en Italie. Aspects sociaux et culturels aux deux premiers siècles de l'Empire (Bibl. des Écoles Fr. d'Athènes et de Rome 364), Rome 2015.

Gummerus, Ärztestand = H. G. Gummerus, Der Ärztestand im römischen Reiche nach den Inschriften (Soc. Scient. Fennica, Comment. Human. Litt. 3, 6), Helsingfors 1932.

Guzmán Almagro, La tradició de l'epigrafia romana $=\mathrm{A}$ Guzmán Almagro, La tradició de l'epigrafia romana al Renaixement. Patrimoni bibliogràfic català (Textos i Estudis de Cultura Catalana 130), Montserrat 2008.

HAACK, Haruspices = M.-L. HAACK, Les haruspices dans le monde romain (Scripta antiqua 6), Bordeaux 2003.

HaAck, Prosopographie = M.-L. HaAck, Prosopographie des haruspices romains (Biblioteca di "Studi Etruschi" 42), Pisa - Roma 2006

HäLVÄ-NYBerg, Kontraktionen = U. HäLVä-NYBERG, Die Kontraktionen auf den lateinischen Inschriften Roms und Afrikas bis zum 8. Jh. n. Chr. (Annales Acad. Scient. Fennicae, Diss. Human. Litt. 49), Helsinki 1988.

HäNLEIN-SCHäFER, Veneratio Augusti = $\mathrm{H}$. HäNLEINSCHÄFer, Veneratio Augusti. Eine Studie zu den Tempeln des ersten römischen Kaisers (Archaeologica 39), Roma 1985.

HaHn, Franen = U. HaHn, Die Frauen des römischen Kaiserhauses und ihre Ebrungen im griechischen Osten anhand epigraphischer und numismatischer Zengnisse von Livia bis Sabina (Saarbrücker Studien zur Archäologie und alten Geschichte 8), Saarbrücken 1994.

Halfmann, Senatoren $=$ H. Halfmann, Die Senatoren aus dem östlichen Teil des Imperium Romanum bis zum Ende des 2. Jahrbunderts n. Chr. (Hypomnemata 58), Göttingen 1979.

HaLkin, Les esclaves publics = L. HaLKIn, Les esclaves publics chez les Romains, Bruxelles 1897 (lucis ope iterum impressum Roma 1965).
Handbook = Chr. BruUn - J. Edmondson (Ed.), The Oxford Handbook of Roman Epigraphy, Oxford 2015.

Handwerk $=$ W. JANSSEN - R. SCHMIDT-WIEgAND - H. TiEFENBACH (Ed.), Das Handwerk in vor- und frühgeschichtlicher Zeit. Teil I. Historische und rechtshistorische Beiträge und Untersuchungen zur Frühgeschichte der Gilde. Bericht über die Kolloquien der Kommission für die Altertumskunde Mittel- und Nordeuropas in den Jabren 1977 bis 1980 (Abh. d. Akad. d. Wiss. Göttingen, Phil.-hist. Kl., 3. 122), Göttingen 1981.

Handwörterbuch = H. HeINEN (†) et AL. (Ed.), Handwörterbuch der antiken Sklaverei I-III (Forsch. Sklaverei 5), Stuttgart 2017.

HARMAND, Le patronat $=$ L. HaRmand, Le patronat sur les collectivités publiques des origines an Bas-Empire: un aspect social et politique du monde romain, Paris 1957.

Harris, Popular Medicine = W. V. Harris (Ed.), Popular Medicine in Graeco-Roman Antiquity: Explorations (Columb. Stud. Class. 42), Leiden - Boston 2016.

Hellenismus I-II = P. ZANKer (Ed.), Hellenismus in Mittelitalien. Kolloquium in Göttingen vom 5. bis 9. Juni 1974 I-II (Abh. d. Akad. d. Wiss. Göttingen, Phil.-hist. Kl., 3. 97), Göttingen 1976.

Helttula, Studies = A. Helttula, Studies on the Latin accusative absolute (Soc. Scient. Fennica, Comment. Human. Litt. 81), Helsinki 1987.

Hemelrijк, Hidden Lives = E. A. Hemelrijk, Hidden Lives, Public Personae. Women and Civic Life in the Roman West, Oxford 2015

Henning, Seianus = D. Henning, L. Aelius Seianus. Untersuchungen zur Regierung des Tiberius (Vestigia 21), München 1975.

Héraclès = C. Bonnet - C. Jourdain-ANNEQuin (Ed.), Héraclès. D'une rive à l'autre de la Méditerranée: bilan et perspectives. Actes de la Table Ronde de Rome, Academia Belgica - École française de Rome, 15-16 septembre 1989 à l'occasion du Cinquantenaire de l'Academia Belgica, en Hommage à Franz Cumont, son premier Président (Inst. Hist. Belge de Rome. Études 28), Bruxelles 1992.

Herrmann-Otto, Untersuchungen = E. Herrmann-Otto, Ex ancilla natus. Untersuchungen $z u$ den hausgeborenen Sklaven und Sklavinnen im Westen des römischen Kaiserreiches (Forsch. Sklaverei 24), Stuttgart 1994.

Hiberia - Italia = A. SARTORI - A. VALvo (Ed.), Hiberia - Italia. Italia - Hiberia. Convegno internazionale di Epigrafia e Storia Antica, Gargnano - Brescia, 28-30 aprile 2005, Peschiera Borromeo (MI) 2006.

Historia Augusta Colloquium 1971 = J. StRAuB (Ed.), Bonner Historia-Augusta-Colloquium 1971 (Antiquitas, Reihe 4. Beiträge zur Historia-Augusta-Forschung 11), Bonn 1974.

HoAre, Classical tour $=$ R. C. HoAre, A classical tour through Italy and Sicily I-II, London 1819.

Höbenreich, Annona = E. HöBenreich, Annona. Juristische Aspekte der stadtrömischen Lebensmittelversorgung im Prinzipat (Grazer Rechts- und Staatswissenschaftliche Studien 55), Graz 1997.

Нøјте, Roman Imperial Statue = J. М. Нøјте, Roman Imperial Statue Bases from Augustus to Commodus (Aarhus Studies in Mediterranean Antiquity 7), Aarhus 2005.

Hörig - SChwertheim, Corpus = M. Hörig - E. SChwerTHEIM, Corpus Cultus Iovis Dolicheni (EPRO 106), Leiden - New York - Københaven - Köln 1987.

Hoffman Lewis, Official Priests = M. W. Hoffman Lewis, The Official Priests of Rome under the Julio-Claudians. A Study of the Nobility from 44 B. C. to 68 A. D. (Pap. Mon. Am. Acad. Rome 16), Rome 1955.

Hofmann, Stand und Aufgaben = J. B. Hofmann (Ed.), Stand und Aufgaben der Sprachwissenschaft. Festschrift für Wilhelm Streitberg, Heidelberg 1924. 
A. Holder, Sprachschatz I-III = A. Holder, Alt-celtischer Sprachschatz I-III, Leipzig 1896/1913.

P. A. Holder, Auxilia = P. A. Holder, Studies in the Auxilia of the Roman Army from Augustus to Trajan (BAR. International Series 70), Oxford 1980.

Holstenius, ms. $\mathrm{I}=\mathrm{L}$. Holstenius, emendationes in $D e$ Teate antiquo, Romae 1651, auctore LuCIO CAMARRA, servatae in codice Bibliothecae Apostolicae Vaticanae sign. Barb. lat. 2291, f. 262r-274v, a. 1647 a LuCA Holstenio exaratae.

Holstenius, $m s$. II $=$ L. Holstenius, Via Tiburtina, Valeria, Nomentana, Salaria, Praenestina, Lavicana, Latina. Iter a. $1627 / 30$ et 1649 a LuCA HolsTENIO factum per vias supra memoratas servatum Dresden in Sächsische Landesbibliothek - Staats- und Universitätsbibliothek ms. sign. n. 193 de quo vide MommSen p. XLV (de re universa nunc vide $\mathrm{L}$. Benedetti, QFIAB 94, 2014, 166-218; Eund., in Peregrinationes 291-318).

Homenaje Armin U. Stylow = Espacios, usos y formas de la epigrafía hispana en épocas antigua y tardoantica. Homenaje al Dr. Armin U. Stylow (Anejos de Archivo Español de Arqueología 48), Mérida 2009.

Homenaje José Remesal Rodríguez = V. Revilla Calvo et AL. (Ed.), Ex Baetica Romam. Homenaje a José Remesal Rodríguez (Col-lecció Homenatges 58), Barcelona 2019.

Homenaje Ricardo Olmos = P. BÁdenas de la Peña - P. Cabrera Bonet - M. Moreno Conde - A. Ruiz RodríGUEZ - C. SÁnchez FernándeZ - T. Tortosa Rocamora (Ed.), Per speculum in aenigmate. Homenaje a Ricardo Olmos. Miradas sobre la Antigüedad (Erytheia. Estudios y Textos 7), Madrid 2014.

Homines de Carapellas $=$ Homines de Carapellas. Storia e archeologia della Baronia di Carapelle (DASP. Studi e testi 10), L'Aquila 1988.

Hommages Carl Deroux I-IV = P. Defosse (Ed.), Hommages à Carl Deroux I-IV (Coll. Latomus 266-267. 270. 277), Bruxelles 2002/03.

Hommages Francis Tassaux = A. Bouet - C. Petit-Aupert (Ed.), Bibere, ridere, gaudere, studere, hoc est vivere. Hommages à Francis Tassaux (Ausonius publications. Mémoires 9), Bordeaux 2018

Honroth, Stadtrömische Girlanden = M. НоNROTH, Stadtrömische Girlanden: ein Versuch zur Entwicklungsgeschichte römischer Ornamentik (Österreichisches Archäologisches Institut, Sonderschriften 17), Wien 1971.

Hoogma, Der Einfluss Vergils = P. Hoogma, Der Einfluss Vergils auf die Carmina Latina Epigraphica. Eine Studie mit besonderer Berücksichtigung der metrisch-technischen Grundsätze der Entlehnung, Amsterdam 1959.

HORSTER, Bauinschriften $=$ M. HORSTER, Bauinschriften römischer Kaiser. Untersuchungen zu Inschriftenpraxis und Bautätigkeit in Städten des westlichen Imperium Romanum in der Zeit des Prinzipats (Historia Einzelschriften 157), Stuttgart 2001.

Howald - Meyer, Römische Schweiz = E. Howald - E. MeYER, Die römische Schweiz. Texte und Inschriften mit Übersetzung, Zürich [1940]

HÜBNER, Exempla $=$ AE. HüBNER, Exempla scripturae epigraphicae Latinae a Caesaris dictatoris morte ad aetatem Iustiniani (CIL Auctarium), Berolini 1885.

HütTl, Antoninus Pius I-II = W. HütTL, Antoninus Pius I. Historisch-politische Darstellung, Prag 1936. II. Römische Reichsbeamte und Offiziere unter Antoninus Pius. Antoninus Pius in den Inschriften seiner Zeit, Prag 1933.

Humbert, Municipium = M. Humbert, Municipium et civitas sine suffragio. L'organisation de la conquête jusqu'à la guerre sociale (Coll. de l'École Fr. de Rome 36), Paris Roma 1978.
HurLEt, Les collègues du prince $=\mathrm{F}$. HuRLET, Les collègues $d u$ prince sous Auguste et Tibère. De la légalité républicaine à la légitimité dynastique (Coll. de l'École Fr. de Rome 227), Paris - Roma 1997.

Hurlet, Proconsul $=$ F. Hurlet, Le proconsul et le prince d'Auguste à Diocletien (Scripta antiqua 18), Bordeaux 2006.

IAnnacchino, Telesia $=$ M. A. IAnnacchino, Storia di Telesia. Sua diocesi e pastori, Benevento 1900.

Iannacchino, Topografia $=$ M. A. IAnnacchino, Topografia storica dell'Irpinia I-IV, Napoli 1889/94.

Iasiello, Samnium = I. M. IAsiello, Samnium. Assetti e trasformazioni di una provincia dell'Italia tardoantica (Pragmateiai 11), Bari 2007.

Iconographie du quotidien = S. LeVEBVRE (Ed.), Iconographie $d u$ quotidien dans l'art provincial romain: modèles régionaux. Actes du XIV veme Congrès international d'art provincial romain, Dijon, juin 2015 (Revue archéologique de l'Est. Supplement 44), Dijon 2017.

$I C V R=$ Inscriptiones Christianae urbis Romae saec. VII antiquiores I. Ed. G. B. DE Rossi, Roma 1857/61. - Supplementum. Ed. G. GATTI, Roma 1915 [cf. infra inter series breviate laudatos p. CLXXV].

Identità e autonomie = A. SARTORI - A. VALVo (Ed.), Identità e autonomie nel mondo romano occidentale. Iberia-Italia - Italia-Iberia. III Convegno Internazionale di Epigrafia e Storia Antica, Gargnano, 12-15 maggio 2010 (Epigrafia e Antichità 29), Bologna - Faenza 2011.

Identità e civiltà dei Sabini = Identità e civiltà dei Sabini. Atti del XIII Convegno di Studi etruschi ed italici, Rieti Magliano Sabina, 30 maggio-3 giugno 1993, Firenze 1996.

IGRR = Inscriptiones Graecae ad res Romanas pertinentes I (et II) - IV. Ed. R. Cagnat, Paris 1906/27 (lucis ope iterum impressum Chicago 1975).

IGUR = L. MORETTI (Ed.), Inscriptiones Graecae urbis Romae I-IV (Stud. Ist. It. Stor. Ant. 17. 22. 28. 47), Romae 1968/ 90.

ILA = Inscriptions Latines d'Aquitaine.

ILAfr $=$ R. CAGNAT - A. MerLin, Inscriptions latines d'Afrique (Tripolitaine, Tunisie, Maroc), Paris 1923.

ILAlg = ST. GselL, Inscriptions latines de l'Algérie, Paris 1922/ 57.

littadino = M. G. Angeli Bertinelli - A. Donati (Ed.), Il cittadino, lo straniero, il barbaro, fra integrazione ed emarginazione nell'antichità. Atti del I Incontro Internazionale di Storia Antica, Genova, 22-24 maggio 2003 (Serta antiqua 8. Storia antica 4), Roma 2005.

IL CV $=$ Inscriptiones Christianae Latinae veteres I-III. Ed. E. DieHL, Berlin 1925/31 (lucis ope iterum impressum Berlin 1961). - IV. Ed. J. Moreau - H. I. Marrou, Dublini Turici 1967.

ILLRP = A. Degrassi (Ed.), Inscriptiones Latinae liberae rei publicae I-II (Biblioteca di Studi Superiori 23. 40), Firenze 1957/63 (I ${ }^{2}$ Firenze 1965, lucis ope iterum impressum Firenze 1972).

ILNarb V.2 Vienne = B. RÉMY et AL., Inscriptions Latines de Narbonnaise V.2 Vienne, Paris 2004.

ILPGranada = M. Pastor Muñoz - A. Mendoza Eguaras (Ed.), Inscripciones Latinas de la Provincia de Granada, Granada 1987.

ILS = H. Dessau (Ed.), Inscriptiones Latinae selectae I-III, Berolini 1892/1916 (lucis ope iterum impressum Berolini 1954/55, Berolini 1962).

IL Sard = G. SotGIU (Ed.), Iscrizioni latine della Sardegna. Supplemento al Corpus inscriptionum Latinarum, $X e$ all'Ephemeris epigraphica, VIII, Padova 1961/68.

Imagines Italicae $=\mathrm{M}$. H. CRAWFORD et AL. (Ed.), Imagines Italicae. A Corpus of Italic Inscriptions (Bull. Inst. Class. Stud. Supplement 110), London 2011. 
Immagini del tempo = Immagini del tempo. La Valle del Sagittario tra natura e cultura. Anversa degli Abruzzi, Cocullo, Villalago, Sambuceto $(\mathrm{CH}) 2002$

Incidenza dell'Antico = L. Breglia PulCi Doria (Ed.), L'incidenza dell'Antico. Studi in memoria di Ettore Lepore. Atti del Convegno Internazionale, Anacapri, 24-28 marzo 1991 I-II, Napoli 1996

Incorrupta antiquitas = Incorrupta antiquitas. Studi di storia, epigrafia e diritto in memoria di Giorgio Luraschi. Atti dell'incontro di studio di Como, 25-26 maggio 2012, Pavia 2017.

In finibus Alifanis = D. CAIAZZA - L. R. Cielo (Ed.), In finibus Alifanis. Storia e archeologia di Alife e del suo distretto, Piedimonte Matese (CE) 2001.

In itinere = F. SIRANO (Ed.), In itinere. Ricerche di archeologia in Campania. Atti del I e del II ciclo di conferenze di ricerca archeologica nell'Alto Casertano, Cava dei Tirreni (SA) 2007.

Inschriftenkulturen $=\mathrm{K}$. BOLLE et AL. (Ed.), Inschriftenkulturen im kommunalen Italien Traditionen, Brüche, Neuanfänge (Materiale Textkulturen 21), Berlin - Boston 2019.

Inscr. Aquil. = J. [= G.] B. Brusin (Ed.), Inscriptiones Aquileiae I-III (Pubblicazioni della Deputazione di Storia patria per il Friuli 20, 1-3), Udine 1991.

Inscriptions in the Private Sphere $=$ R. Benefiel - P. KeEgan (Ed.), Inscriptions in the Private Sphere in the GrecoRoman World (Brill Studies Epigraphy 7), Leiden 2016.

Instrumenta Inscripta III = G. BARATTA - S. M. Marengo (Ed.), Instrumenta Inscripta III. Manufatti iscritti e vita dei santuari in età romana, Gorgonzola (MI) 2012.

Instrumenta Inscripta $\mathrm{V}=\mathrm{A}$. BuONOPANE - S. BRAITO - C. GIRARDI (Ed.), Instrumenta Inscripta V. Signacula ex aere. Aspetti epigrafici, archeologici, giuridici, prosopografici, collezionistici. Atti del convegno internazionale, Verona, 20-21 settembre 2012, Roma 2014.

Interventi $=$ A. STORChI MARINo - G. D. Merla (Ed.), Interventi imperiali in campo economico e sociale. Da Augusto al Tardoantico (Pragmateiai 18), Bari 2009.

Iovenitti, Paganica $=$ E. Iovenitti, Paganica attraverso $i$ secoli, Sulmona (AQ) 1973.

IRT = J. M. Reynolds - J. B. Ward Perkins (Ed.), The Inscriptions of Roman Tripolitania. In collaboration with S. Aurigemma - R. BartocCini - G. CAPUto - R. GoodCHILd - P. Romanelli, Rome - London 1952.

IsABella, Iscrizioni Allifae $=$ C. IsABELLA, Le iscrizioni antiche di Allifae (Libri Campano-Sannitici 6), Piedimonte Matese (CE) 2007.

Iscrizione esposta = A. Donati (Ed.), L'iscrizione esposta. Atti del Convegno Borghesi 2015, Bertinoro, 4-6 giugno 2015 (Epigrafia e Antichità 37), Bologna - Faenza 2016.

Iscrizioni dei cristiani = I. Di Stefano Manzella (Ed.), Le iscrizioni dei cristiani in Vaticano. Materiali e contributi scientifici per una mostra epigrafica (ISS 2), Città del Vaticano 1997.

Isernia $=$ C. Terzani - M. MATTEINI ChIARI (Ed.), Isernia. La necropoli romana in località Quadrella, Tivoli (RM) 1997.

Isis en Occident = L. Bricault (Ed.), Isis en Occident. Actes du II ème Colloque international sur les études isiaques, Lyon III, 16-17 mai 2002 (Religions in the Graeco-Roman World 151), Leiden - Boston 2004

ISS = Inscriptiones Sanctae sedis.

Italia omnium terrarum alumna = Italia omnium terrarum alumna. La civiltà dei Veneti, Reti, Liguri, Celti, Piceni, Umbri, Latini Campani e Iapigi, Milano 1988.

Italia omnium terrarum parens = Italia omnium terrarum parens. La civiltà degli Enotri, Choni, Ausoni, Sanniti, Lucani, Brettii, Sicani, Siculi, Elimi, Milano 1989.
Italica ars = D. CAIAzZA (Ed.), Italica ars. Studi in onore di Giovanni Colonna per il premio "I Sanniti" (Libri Campano-Sannitici 4), Piedimonte Matese (CE) 2005.

Itinera archaeologica $=$ E. МАттIосCо (Ed.), Itinera archaeologica. Contributi di archeologia abruzzese (Novitas 5), Lanciano (CH) 2006

Itinéraire = J.-P. Bost - J.-M. RodDAZ - F. TAssaux (Ed.), Itinéraire de Saintes à Dougga. Mélanges offerts à Louis Maurin (Ausonius publications. Mémoires 9), Bordeaux 2003.

Iuris vincula $=$ Iuris vincula . Studi in onore di Mario Talamanca I-VIII, Napoli 2001.

Invanum I = [E. FABRICOTTI (Ed.)], Iuvanum. Atti del Convegno di Studi, Chieti, maggio 1983 (Diamanti 7), Chieti 1990.

Iuvanum II = Iuvanum. Atti del II Convegno di Studi, Chieti, 31 marzo - 1 aprile 1992, Pescara 1996.

Iuvanum III = S. LAPENNA (Ed.), Iuvanum. L'area archeologica, Sulmona (AQ) 2006

JACOBILLI, $m s . \mathrm{I}=\mathrm{L}$. JACOBILLI, Inscriptiones antiquae existentes in urbibus et locis provinciae Umbriae, Fulginiae 1661 (liber manu scriptus in Biblioteca Jacobilli adservatus Foligno, sub n. B. V. 9).

JaCobilli, $m s$. II = L. Jacobilli, Historia di Norsia, prima Prefettura de' Sabini et ... Municipio de' Romani (liber manu scriptus saec. XVII medio exaratus in Biblioteca Jacobilli adservatus Foligno, sub n. B. IV. 13).

Jасовьон, Altitalische Inschriften $=\mathrm{H}$. Јасовsонn, Altitalische Inschriften (Kleine Texte 57), Berlin 1927.

Jacques, Cités = F. Jacques, Les cités de l'occident romain . Du $I^{e r}$ siècle avant J.-C. au IV siècle après J.-C., Paris 1990.

JACQUES, Curateurs = F. JACQUES, Les curateurs des cités dans l'Occident romain de Trajan à Gallien (Études prosopographiques 5), Paris 1983.

Jacoues, Privilège $=\mathrm{F}$. Jacoues, Le privilège de liberté. Politique impériale et autonomie municipale dans les cités de l'Occident romain (161-244) (Coll. de l'École Fr. de Rome 76), Paris - Rome 1984.

JACZYNOWSKA, Les associations = M. JACZYNowsKA, Les associations de la jeunesse romaine sous le Haut-Empire (Archiwum Filologiczne 36), Wrocław - Warszawa Kraków - Gdańsk 1978.

JOHNE - KÖHN - Weber, Kolonen $=$ K. P. JOHNE - J. KÖHN V. Weber, Die Kolonen in Italien und den westlichen Provinzen des römischen Reiches. Eine Untersuchung der literarischen, juristischen und epigraphischen Quellen vom 2. Jahrbundert v. u. Z. bis zu den Severern (Schriften zur Geschichte und Kultur der Antike 21), Berlin 1983.

JosheL, Work = S. R. JosheL, Work, Identity and Legal Status at Rome. A Study of the Occupational Inscriptions (Oklahoma Series in Classical Culture 11), Norman, OK London 1992.

JOUfFroy, La construction publique $=$ H. JOUfFroy, La construction publique en Italie et dans l'Afrique romaine (Univ. Strasbourg. Etudes et Travaux 2), Strasbourg 1986.

Kajanto, Cognomina = I. Kajanto, The Latin Cognomina (Soc. Scient. Fennica, Comment. Human. Litt. 36, 2), Helsinki 1965.

Kajanto, Onomastic Studies = I. Kajanto, Onomastic Studies in the Early Christian Inscriptions of Rome and Carthage (Acta Inst. Rom. Finl. 2, 1), Helsinki 1963.

Kajanto, Supernomina $=$ I. KaJanto, Supernomina. A Study in Latin Epigraphy (Soc. Scient. Fennica, Comment. Human. Litt. 40, 1), Helsinki 1966.

Kajava, Praenomina = M. Kajava, Roman Female Praenomina. Studies in the Nomenclature of Roman Women (Acta Inst. Rom. Finl. 14), Helsinki - Rome 1994. 
KAN, Iuppiter Dolichenus = A. H. KAN, Iuppiter Dolichenus. Sammlung der Inschriften und Bildwerke mit einer Einleitung, Leiden 1943.

KeIL, Gramm. Lat. = M. KeIL, Grammatici Latini I, Lipsiae 1857.

Keppie, Colonisation $=\mathrm{L}$. KeppIE, Colonisation and veteran settlement in Italy, 4-14 B. C., Rome 1983.

Keppie, Roman Army = L. Keppie, The Making of Roman Army. From Republic to Empire, London 1984.

Kettenhofen, Die syrischen Augustae = E. Kettenhofen, Die syrischen Augustae in der historischen Überlieferung: Ein Beitrag zum Problem der Orientalisierung (Antiquitas 3. 24), Bonn 1979.

KIEnAST, Kaisertabelle ${ }^{6}=$ D. KIENAST, Römische Kaisertabelle. Grundzüge einer römischen Kaiserchronologie. 6. vollständig überarbeitete und aktualisierte Auflage curantibus Werner Eck et Matthäus Heil, Darmstadt 2017.

KIENAST, Kriegsflotten $=$ D. KIENAST, Untersuchungen $z u$ den Kriegsflotten der römischen Kaiserzeit (Antiquitas 1. 13), Bonn 1966

Kleijwegt, Ancient Youth = M. Kleijwegt, Ancient Youth . The Ambiguity of Youth and the Absence of Adolescence in Greco-Roman Society, Amsterdam 1992.

KolB, Curatores = A. KolB, Die kaiserliche Bauverwaltung der Stadt Rom. Geschichte und Aufbau der cura operum publicorum unter dem Prinzipat (HABES 13), Stuttgart 1993.

KonRad, Plutarch's Sertorius = C. F. KonRad, Plutarch's Sertorius. A historical Commentary, Chapel Hill - London 1994.

Kovács, Pannonia $=$ P. Kovács, $A$ History of Pannonia during the Principate (Antiquitas 1. 65), Bonn 2014.

KRAFT, Rekrutierung $=\mathrm{K}$. KRAFT, Zur Rekrutierung der Alen und Kohorten an Rhein und Donau (Dissertationes Bernenses 3), Bern 1951.

KRAHE, Personennamen $=\mathrm{H}$. KRAHE, Lexikon altillyrischer Personennamen (Indog. Bibl. 3. 9), Heidelberg 1929.

KreILer, Statthalter $=$ B. KreILer, Die Statthalter Kleinasiens unter den Flaviern, Diss. München 1975.

Kruschwitz, Carmina = P. Kruschwitz, Carmina Saturnia Epigraphica. Einleitung, Text und Kommentar zu den saturnischen Versinschriften (Hermes Einzelschriften 84), Stuttgart 2002.

KRUSCHWITZ, Römische Inschriften = P. KRUSCHWITZ, Römische Inschriften und Wackernagels Gesetz. Untersuchungen zur Syntax epigraphischer Texte aus republikanischer Zeit (Schriften der Philosophisch-historischen Klasse der Heidelberger Akademie der Wissenschaften 31), Heidelberg 2004.

Kubitscheк, Imperium $=\mathrm{J}$. W. Kubitscheк, Imperium Romanum tributim descriptum, Pragae - Vindobonae Lipsiae 1889.

Kulte - Riten $=$ P. AMANN - E. Bruna (Ed.), Kulte - Riten religiöse Vorstellungen bei den Etruskern und ihr Verbältnis zu Politik und Gesellschaft. Akten der 1. Internationalen Tagung der Sektion Wien/Osterreich des Istituto Nazionale di Studi Etruschi ed Italici (Wien, 4.-6, 12. 2008) (Österr. Akad. Wiss. Phil.-Hist. Klasse. Denkschriften 440), Wien 2012

LACAM, Variations rituelles $=\mathrm{J}$. -C. LACAM, Variations rituelles. Les pratiques religieuses en Italie centrale et méridionale an temps de la deuxième Guerre Punique (Coll. de l'École Fr. de Rome 430), Rome 2010.

La commemorazione di Germanico = A. FrasCHetTI (Ed.), La commemorazione di Germanico nella documentazione epigrafica. Tabula Hebana e Tabula Siarensis. Convegno internazionale di studi, Cassino, 21-24 ottobre 1991 (Saggi di storia antica 14), Roma 2000
La cultura classica a Napoli $=$ La cultura classica a Napoli nell'Ottocento. Secondo contributo. Premessa di M. Gigante (Pubblicazioni del Dipartimento di Filologia Classica dell'Università degli Studi di Napoli "Federico II" 4 [2]), Napoli 1991.

LAFER, Omnes collegiati $=$ R. LAFER, Omnes collegiati, "concurrite"! Brandbekämpfung im Imperium Romanum (Grazer Altertumskundliche Studien 7), Frankfurt am Main [u. a.] 2001.

Laffi, Adtributio = U. Laffi, Adtributio e contributio. Problemi del sistema politico-amministrativo dello stato romano (Studi di lettere, storia e filosofia della Scuola Normale di Pisa 28), Pisa 1966.

Laffi, Colonie $=$ U. LafFI, Colonie e municipi nello stato romano (SL Raccolta di studi e testi 239), Roma 2007.

LAFFI, Studi = U. LAFFI, Studi di storia romana e di diritto (SL Raccolta di studi e testi 206), Roma 2001.

Lago Fucino = E. Burri (Ed.), Il proscingamento del Lago Fucino e l'emissario sotterraneo, Pescara 2011.

LaIrd, Civic Monuments = M. L. LAIRD, Civic Monuments and the Augustales in Roman Italy, Cambridge 2015.

Lalli, Miscellanea $=$ C. Lalli, Miscellanea di notizie storiche e cronache riguardanti Norcia (schedae epigraphicae saec. XX in. manu exaratae servatae Viterbo penes heredes Lalli; quarum copia servatur Spoleto apud Romano CoRdella, qui me certiorem fecit).

Lalli, $m s .=$ C. Lalli, Annotazioni varie riguardanti l'antiche età di Norcia e la copia fedele di molti frammenti epigrafici romani ivi esistenti (liber circ. a. 1920 manu exaratus in bibliotheca communali oppidi Norcia olim adservatus sine inv. n.; postquam terrae motus d. 24 m. Aug. et d. 30 m. Oct. a. 2016 universum oppidum diruerunt, Spoleto est translatus).

Lambrechts, Composition du Sénat $=$ P. Lambrechts, $L a$ composition du Sénat roman de l'accession au trône de Hadrien à la mort de Commode (117-192), Antwerpen Paris 1936.

La mort, les morts = P. HinARD (Ed.), La mort, les morts et l'au-delà dans le monde romain. Actes du colloque de Caen, 20-21 Novembre 1985, Caen 1987.

LANCIANI, $m s .=$ R. LANCIANI, Libri saec. XIX ex. - XX in. manu scripti in Bibliotheca Apostolica Vaticana adservati sign. Vaticani Latini 13031-13047 (vide supra BuonoCORE, Appunti I-V).

LanCIANI, Acque = R. LANCIANI, Le acque e gli acquedotti $d i$ Roma antica, Roma 1975 (sub titulo Topografia di Roma antica: I comentarii di Frontino intorno le acque e gli aquedotti, Roma 1881 = Memorie della Reale Accademia dei Lincei. Classe di scienze morali 3, 4, Roma 1880, 215616).

Lanciani, Commentarii $=$ R. Lanciani, $I$ Commentarii $d i$ Frontino intorno le acque e gli acquedotti, Roma 1881.

Lanzellotti, Antichità teatine = B. Lanzellotti, Antichità teatine. Memorie, Teramo 1891.

LanzellotTi, Antico sepolcreto = B. LANZellotti, $D i$ un antico sepolcreto presso Chieti, Chieti 1882.

Lanzellotti, Della vita $=$ B. Lanzellotti, Della vita e degli studi di Cajo Asinio Pollione Marrucino, Prato 1875.

LANZONI, Le diocesi d'Italia = F. LANZONI, Le diocesi d'Italia dalle origini al principio del secolo VII (an. 604) (BAV. Studi e testi 35), Bologna - Faenza 1927.

Lapidario Zeri = G. BARBIERI et AL., Il lapidario Zeri di Mentana I-II (Stud. Ist. It. Stor. Ant. 32), Roma 1982.

La question agraire à Rome $=\mathrm{E}$. HeRMON (Ed.), La question agraire à Rome: droit romain et société. Perceptions historiques et historiographiques (Biblioteca di Athenaeum 44), Como 1999.

L'art décoratif $=$ L'art décoratif à Rome à la fin de la Republique et au début du Principat. Table ronde organisée par 
l'École française de Rome, Rome, 10-11 mai 1979 (Coll. de l'École Fr. de Rome 55), Rome 1981.

LAssére, Manuel $^{2}$ I-II = J.-M. LAssére, Manuel d'épigraphie romaine ${ }^{2} \mathrm{I}-\mathrm{II}$, Paris 2007

La terza età $=$ A. Donati (Ed.), La terza età dell'epigrafia. Atti del Convegno AIEGL - Borghesi 86, Bologna, ottobre 1986 (Epigrafia e Antichità 9), Bologna - Faenza 1988.

Latin vulgaire VI $=\mathrm{H}$. Halla-AHO - M. LeIwo $-\mathrm{H}$. Solin (Ed.), Latin vulgaire - latin tardif. Actes $d u V^{e}$ colloque international sur le latin vulgaire et tardif, Helsinki, 29 août - 2 septembre 2000, Hildesheim - New York - Zürich 2003.

Latin vulgaire VII = C. ARIAS ABELLÁN (Ed.), Latin vulgaire latin tardif. Actes du VII ème Colloque international sur le latin vulgaire et tardif, Séville, 2-6 septembre 2003 (Colección Actas 54), Sevilla 2006.

LATTE, Religionsgeschichte $=\mathrm{K}$. LATTE, Römische Religionsgeschichte (Handbuch der Altertumswissenschaft 5, 4), München 1960.

LATTIMORE, Themes $=$ R. LATTIMORE, Themes in Greek and Latin Epitaphs (Illin. Studies 28), Urbana Illin. 1942.

Laum, Stiftungen $=$ B. Laum, Stiftungen in der griechischen und römischen Antike, Leipzig - Berlin 1914.

Lazio \& Sabina I = J. Rasmus Brandt - X. Dupré Raventós - G. Ghini (Ed.), Lazio \& Sabina. Atti del Convegno. Primo Incontro di Studi sul Lazio e la Sabina, Roma, 28 30 gennaio 2002 (Lavori e Studi 1), Roma 2003.

Lazio \& Sabina II = G. GHINI (Ed.), Lazio \& Sabina. Atti del Convegno. Secondo Incontro di Studi sul Lazio e la Sabina, Roma, 7-8 maggio 2003 (Lavori e Studi 2), Roma 2004.

Lazio \& Sabina III = G. GHINI (Ed.), Lazio \& Sabina. Atti del Convegno. Terzo Incontro di Studi sul Lazio e la Sabina, Roma, 18-20 novembre 2004 (Lavori e Studi 3), Roma 2006.

Lazio \& Sabina IV = G. GhinI (Ed.), Lazio \& Sabina. Atti del Convegno. Quarto Incontro di Studi sul Lazio e la Sabina, Roma, 29-31 maggio 2006 (Lavori e Studi 4), Roma 2007.

Lazio \& Sabina V = G. GHINI (Ed.), Lazio \& Sabina. Scoperte, scavi e ricerche. Atti del Convegno. Quinto Incontro di Studi sul Lazio e la Sabina, Roma, 3-5 dicembre 2007 (Lavori e Studi 5), Roma 2009.

Lazio \& Sabina VI = G. GHINI (Ed.), Lazio \& Sabina. Atti del Convegno. Sesto Incontro di Studi sul Lazio e la Sabina, Roma, 4-6 marzo 2009 (Lavori e Studi 6), Roma 2010.

Lazio \& Sabina VII = G. GHINI (Ed.), Lazio \& Sabina. Atti del Convegno. Settimo Incontro di Studi sul Lazio e la Sabina, Roma, 9-11 marzo 2010 (Lavori e Studi 7), Roma 2011.

Lazio \& Sabina VIII = G. GHINI - Z. MARI (Ed.), Lazio \& Sabina. Atti del Convegno. Ottavo Incontro di Studi sul Lazio e la Sabina, Roma, 30-31 marzo, 1 aprile 2011 (Lavori e Studi 8), Roma 2012.

Lazio \& Sabina IX = G. GhinI - Z. Mari (Ed.), Lazio \& Sabina. Atti del Convegno. Nono Incontro di Studi sul Lazio e la Sabina, Roma, 27-29 marzo 2012 (Lavori e Studi 9), Roma 2013.

Lazio \& Sabina X = A. Russo TAgliente - G. Ghini - Z. Mari (Ed.), Lazio \& Sabina. Atti del Convegno. Decimo Incontro di Studi sul Lazio e la Sabina, Roma, 4-6 giugno 2013 (Lavori e Studi 10), Roma 2016.

Lazio \& Sabina XI = A. Russo TAgLiente - G. GHINI - Z. Mari (Ed.), Lazio \& Sabina. Atti del Convegno. Undicesimo Incontro di Studi sul Lazio e la Sabina, Roma, 4-6 giugno 2014 (Lavori e Studi 11), Roma 2016.

LAZZARINI, Sepulcra familiaria $=$ S. LAZZARINI, Sepulcra familiaria. Un'indagine epigrafico-giuridica (Pubblicazioni dell'Università di Pavia. Studi delle scienze giuridiche e sociali, n. s. 65), Padova 1991.
Le Bohec, Imperial Roman Army = Y. Le BoHec, The Imperial Roman Army, London 1994 (editio Anglica libri LE BoHEC, L'armée romaine).

Le BoHec, L'armée romaine $=\mathrm{Y}$. LE BoHec, L'armée romaine sous le Haut-Empire, Paris 1984.

Lege nunc, viator $=$ N. CRINITI (Ed.), Lege nunc, viator ...: vita e morte nei carmina Latina epigraphica della Padania centrale (Bellissime navigare 1), Parma 1996.

Légions de Rome = Y. LE BoHEC (Ed.), Les légions de Rome sous le Haut-Empire. Actes du Congrès international de Lyon, 17-19 septembre 1998 I-II, Lyon 2000.

Legrottaglie, Sistema delle immagini = G. LegrotTAglie, Il sistema delle immagini negli anfiteatri romani (Beni Archeologici - Conoscenza e Tecnologie. Quaderno 7), Bari 2008.

Leonardis - Marinangeli, Peltuinum = G. Leonardis - G. Marinangeli, Peltuinum Vestinorum, Aquila 1967.

E. Leoni, La Sabina = E. Leoni, La Sabina nella storia di Roma, Roma 1970.

V. LeONI, $m s .=$ V. LeONI, Libellus manu scriptus, cui titulus Giornale degli scavi che si eseguiscono in territorio di S. Vittorino, prov. di Aquila, voc. Torricello. Ottobre Novembre 1915, servatus Chieti in archivo praefecturae antiquitatum sine inv. $\mathrm{n}$.

Leosini, $m s .=$ A. Leosini, Libri manu scripti a. $1875 / 79$ servati Romae apud Archivio Centrale dello Stato (EUR). MPI. Dir. Gen. AABBAA. Antichità e Scavi. I versamento.

LEPPIN, Histrionen = H. LePPIN, Histrionen. Untersuchungen zur sozialen Stellung von Bühnenkünstlern im Westen des römischen Reiches zur Zeit der Republik und des Principats (Antiquitas 1. 41), Bonn 1992.

Le Roux, L'armée romaine $=\mathrm{P}$. Le Roux, L'armée romaine et l'organisation des provinces ibériques d'Auguste à l'invasion de 409 (Publications du Centre Pierre Paris 8), Paris 1982.

Les "bourgeoisies" municipales = M. CÉBEILlaC-GeRvasonI (Ed.), Les "bourgeoisies" municipales italiennes aux $I I^{e}$ et $I^{e r}$ siècles av. J.-C. Centre Jean Bérard, Institut Français de Naples, 7-10 décembre 1981 (Colloques internationaux du Centre National de la Recherche Scientifique 609 [Sciences humaines]), Paris - Naples 1983.

LESQUIER, L'armée romaine $=\mathrm{H}$. LESQUIER, L'armée romaine d'Égypte d'Auguste à Dioclétien, Le Caire 1918.

LETTA, I Marsi e il Fucino = C. LETTA, I Marsi e il Fucino nell'antichità (CeSDIR. Monografie a supplemento degli "Atti" 3), Milano 1972

Leumann, Laut- und Formenlehre ${ }^{2}=$ M. Leumann, Lateinische Grammatik 1. Lateinische Laut- und Formenlehre ${ }^{2}$ (Handbuch der Altertumswissenschaft 2. 2. 1), München 1977.

Leunissen, Konsuln = P. M. M. Leunissen, Konsuln und Konsulare in der Zeit von Commodus bis Severus Alexander (180-235 n. Chr.). Prosopographische Untersuchungen zur senatorischen Elite im römischen Kaiserreich (Dutch Monographs on Ancient History and Archeology 6), Amsterdam 1989

Lexicon Topographicum I-VI = E. M. STEINBy (Ed.), Lexicon Topographicum urbis Romae I-VI, Roma 1993/2000.

Lexicon Topographicum. Suburbium I-V = A. LA Regina (Ed.), Lexicon Topographicum urbis Romae. Suburbium IV, Roma 2001/08.

Liberatore, Alba Fucens = D. Liberatore, Alba Fucens. Studi di storia e di topografia (Insulae Diomedeae 3), Bari 2004.

Libitina = Libitina e dintorni. Libitina e $i$ luci sepolcrali. Le leges libitinariae campane. Iura sepulcrorum: vecchie e nuove iscrizioni. Atti dell'XI Rencontre franco-italienne sur l'épigraphie, Roma, 10-12 maggio 2002 (Libitina 3), Roma 2004. 
Lieux sacrés = A. VAUCHEZ (Ed.), Lieux sacrés, lieux de culte, sanctuaires. Approches terminologiques, méthodologiques, historiques et monographiques (Coll. de l'École Fr. de Rome 273), Rome 2000.

LIMC = Lexicon Iconographicum Mythologiae Classicae, Zürich 1981/2009.

LiNDERSKI, Roman Questions I = J. LiNDERSKI, Roman Questions I. Selected Papers 1958-1993 (HABES 20), Stuttgart 1995.

LINDERSKI, Roman Questions II = J. LINDERSKI, Roman Questions II. Selected Papers (HABES 44), Stuttgart 2007.

LiNDSAY, Handbook = W. M. LINDSAY, Handbook of Latin Inscriptions Illustrating the History of the Language, Boston - Chicago 1897.

Linguistica storica $=\mathrm{S}$. C. TRоvaто (Ed.), Linguistica storica e dialettologia. Atti del Convegno della Società Italiana di Glottologia, Catania, 3-5 ottobre 2002, Roma 2004.

Liou, Praetores = B. Liou, Praetores Etruriae XV populorum (Étude d'épigraphie) (Coll. Latomus 106), Bruxelles 1969.

L'iscrizione e il suo doppio = A. Donati (Ed.), L'iscrizione e il suo doppio. Atti del Convegno Borghesi 2013, Bertinoro, 6-8 giugno 2013 (Epigrafia e Antichità 35), Bologna Faenza 2014.

L'Italie d'Auguste à Dioclétien = L'Italie d'Auguste à Dioclétien. Actes du colloque international de Rome, 25-28 mars 1992 (Coll. de l'École Fr. de Rome 198), Paris Roma 1994.

Liu, Collegia Centonariorum = J. Liu, Collegia Centonariorum. The Guilds of Textile Dealers in the Roman West (Columb. Stud. Class. 34), Leiden - Boston 2009.

Lo CAScio, Il princeps e il suo impero = E. Lo CASCIO, Il princeps e il suo impero. Studi di storia amministrativa e finanziaria romana (Documenti e studi 26), Bari 2000.

Loffreda, Sannio Pentro = D. Loffreda, Sannio Pentro Alifano II, Napoli 2003.

Lolli, $m s .=$ F. Lolli, Adversaria manu scripta saec. XIX ex. - XX in. servata Romae apud Archivio Centrale dello Stato. MPI. Dir. Gen. AABBAA. Antichità e Scavi.

Lombardi, San Potito Sannitico = N. Lombardi, San Potito Sannitico, Piedimonte Matese (CE) [2004].

L'ombelico d'Italia = A. M. DolciotTi - C. SCARdazza (Ed.), L'ombelico d'Italia. Popolazioni preromane dell'Italia centrale. Atti del Convegno "IV Giornata per l'Archeologia", Roma, 17 maggio 2005, Roma 2007.

L'onomastique latine $=\mathrm{N}$. Duval $-\mathrm{D}$. Briquel $-\mathrm{M}$. Hamiaux (Ed.), Actes du colloque international sur l'onomastique latine organisé à Paris du 13 au 15 octobre 1975 par H.-G. Pflaum et N. Duval (Colloques internationaux $d u$ Centre National de la Recherche scientifique 564), Paris 1977.

L'ordre équestre $=\mathrm{S}$. Demougin - H. Devijver $(\dagger)-$ M.-Th. RAepsaet-Charlier (Ed.), L'ordre équestre. Histoire d'une aristocratie (II ${ }^{e}$ siècle av. J.-C. - III esiècle ap. J.-C.). Actes du colloque international, Bruxelles - Leuven, 5-7 octobre 1995 (Coll. de l'École Fr. de Rome 257), Rome 1999.

Lorenzetti, Studio = A. Lorenzetti, Lo studio delle antichità romane nell'Abruzzo del Seicento: Lucio Camarra, "De Teate antiquo" (1651) (liber machina dactylographica redactus thesis doctoris dignitatis nominisque a. 2004/05 Romae impetratae apud Università degli Studi di Roma "La Sapienza").

Los lugares de la Historia = J. M. Aldea Celada et Al. (Ed.), Los lugares de la Historia. Actas IV Congreso AJHIS, Salamanca 2013 (Colección Temas y Perspectivas de la Historia 3), Salamanca 2013

LuCCI - Di FranCESCANTONIO, Inventarium ms. = F. LuCCI C. Di Francescantonio, Oggetti scavati nel territorio di Corfinio e consegnati al Museo Corfiniese (inventarium a. 1934/76 manu scriptum in Museo Civico Archeologico 'Antonio De Nino' in bibliotheca sine inv. n. Corfinio servatum).

Ludi Trebulani = G. Alvino (Ed.), Ludi Trebulani. L'anfiteatro di Monteleone Sabino e il suo contesto archeologico, Roma - Ostia Antica (RM) 2003

Lugini, Memorie storiche $=$ D. Lugini, Memorie storiche della regione Equicola, ora Cicolano, Rieti 1907 (ed. anastat. Rieti 1983).

LugLI, $m s .=$ G. LugLI, Adversaria ante secundum bellum inter omnes gentes composita, quibus nomen Sabini-Aequi, servata in Biblioteca Romana Sarti dell'Accademia Nazionale di San Luca sub n. cartella 1 sine autem nota foliorum.

LukITs, Schiedsspruch = R. LukITs, Der Schiedsspruch des C. Helvidius Priscus (CIL IX 2827) (Linzer Archäologische Forschungen. Sonderheft 51), Linz 2015.

L'Umbria = L'Umbria I. La Valnerina. Il Nursino. Il Casciano, Roma 1977.

Luoghi degli dei = A. Campanelli - A. Faustoferri (Ed.), I luoghi degli dei. Sacro e natura nell'Abruzzo italico, Sambuceto (CH) 1997.

L'Urbs = L'Urbs. Espace urbaine et histoire (I ${ }^{\text {er }}$ siècle av. J.-C. - III siècle ap. J.-C.). Actes du colloque international organisé par le Centre National de la Recherche Scientifique et l'École Française de Rome, Rome, 8-12 mai 1985 (Coll. de l'École Fr. de Rome 98), Paris - Rome 1987.

MacCrum - Woodhead, Select Documents = M. MacCrum - A. G. Woodhead, Select Documents of the Principates of the Flavian Emperors Including the Year of Revolution A. D. 68-96, Cambridge 1961.

Maciocia, Civita D'Antino = S. Maciocia, Civita D'Antino. Storia, arte, leggende. A cura dell'Associazione Pro-Loco di Civita D’Antino, [Roma] 1956.

Madonna, Iuvanum = A. Madonna, Iuvanum, Roma [1930]. MAfFeI, Museum Veronense = F. Sc. MAFfeI, Museum Veronense. Hoc est antiquarum inscriptionum atque anaglyphorum collectio cui Taurinensis adiungitur et Vindobonensis. Accedunt monumenta id genus plurima nondum vulgata, et ubicumque collecta, Veronae 1749.

MAffei, Tre lettere = F. Sc. MAffeI, Tre lettere ..., Veronae 1748.

Magioncalda, Documentazione epigrafica $=$ A. MagionCALDA, Documentazione epigrafica e 'fondazioni' testamentarie: appunti di una scelta di testi, Torino 1994.

Magioncalda - Christol, Studi = A. Magioncalda - M. Christol, Studi sui procuratori delle due Mauretanie (Pubblicazioni del Dipartimento di Storia dell'Università degli Studi di Sassari 13), Sassari 1989.

Magister = G. FIRPo - G. ZeCCHINI (Ed.), Magister: aspetti culturali e istituzionali. Atti del Convegno, Chieti, 13-14 novembre 1997 (Dipartimento di scienze dell'Antichità. Università degli Studi “G. D’Annunzio”, Chieti. Sezione di storia 2), Alessandria 1999.

Malaise, Conditions = M. Malaise, Les conditions de pénétration et de diffusion des cultes égyptiens en Italie (EPRO 22), Leiden 1972.

Malaise, Inventaire = M. Malaise, Inventaire préliminaire des documents égyptiens découverts en Italie (EPRO 21), Leiden 1972.

Mancini, Allifae $^{1}=$ N. Mancini, Allifae ${ }^{1}$, Piedimonte Matese (CE) 1993.

Mancini, Allifae ${ }^{2}=$ N. MAnCini, Allifae ${ }^{2}$, Brescia 2000.

Mancini, Allifae $e^{3}=$ N. Mancini, Allifa $e^{3}$, Piedimonte Matese (CE) 2005.

Mancini, Articoli $=$ N. Mancini, Articoli. Pubblicati dall'anno 1968 all'anno 2011, Roma 2011 (ed. altera Articoli. Pubblicati dal 1968 al 2014, Roma 2015).

Mancini, Raviscanina. Archivio = N. Mancini, Raviscanina. Archivio fotografico, Roma 2010. 
Mancini, Raviscanina. Ricerche ${ }^{2}=$ N. MANCINI, Raviscanina Ricerche storiche ${ }^{2}$, Roma 2010.

MANCINI - MAstrodDI, Viaggio antiquario = V. MANCINI A. Mastrodd, Viaggio antiquario dei Deputati Sig. D. Vincenzo Mancini e Sig. D. Alessandro Mastroddi (breviarium d. 30 m. Apr. a. 1835 manu scriptum, in quo tituli nonnulli haud semper recte sunt recogniti, servatum $L^{\prime}$ Aquila in archivo rei publicae signatum Intendenza $1^{\circ}$ serie, Cat. 19, b. 4590 publici iuris factum a S. SegENNI, in Fucino III, 375-385. Nunc vide F. Amiconi (Ed.), Viaggio antiquario nella Marsica. Relazione dei monumenti di antichità eseguita da Alessandro Mastroddi e Vincenzo Mancini il 30 aprile 1835, Cerchio - Avezzano (AQ) 2018).

Mander, Portraits $=\mathrm{J}$. Mander, Portraits of Children on Roman Funerary Monuments, Cambridge 2013.

Manni, Storia dei municipi = E. Manni, Per la storia dei municipi fino alla guerra sociale (Stud. Ist. It. Stor. Ant. 5), Roma 1947.

Marcangeli, $m s .=$ G. A. Marcangeli, epistula de titulis nonnullis Carsiolanis missa ad praefectum civitatis L'Aquila d. 11 m. Aug. a. 1879 servata L'Aquila in archivo rei publicae signata Prefettura $1^{\circ}$ serie, Affari Generali, Cat. 14, b. 1, versamento 14, a. 1877-1885. Gratias ago Cesare Castellani, qui me certiorem fecit.

Marganelli, Goriano Sicoli $=$ E. Marganelli, Storia $d i$ Goriano Sicoli, Sulmona (AQ) 1981.

MARINI, $m s .=$ G. MARINI, Libri manu scripti saec. XVIII p. m. in Bibliotheca Apostolica Vaticana adservati sign. Vaticani Latini 9020-9151. 15293 (vide supra Gaetano Marini).

Marinucci, Iscrizioni ${ }^{1}=$ A. Marinucci, Le iscrizioni del Gabinetto Archeologico di Vasto ${ }^{1}$ (Documenti 4), Roma 1974.

Marinucci, Iscrizioni ${ }^{2}=$ A. Marinucci, Le iscrizioni del Gabinetto Archeologico di Vasto ${ }^{2}$ (Documenti 4), Roma 1981.

Marinucci de' Reguardati, $S$. Benedetto = C. Marinucci DE' Reguardati, S. Benedetto di origine Anicia?, Subiaco (RM) 1981

Marocco, Monumenti = G. Marocco, Monumenti dello Stato Pontificio e relazione topografica di ogni paese IXIV, Roma 1833/37.

D. B. Marrocco, Alife = D. B. Marrocco, L'antica Alife, Piedimonte d'Alife (CE) 1951

D. B. Marrocco, Breve storia = D. B. Marrocco, Breve storia dell'epigrafia alifana, Piedimonte d'Alife (CE) 1959

D. B. Marrocco, Piedimonte $=$ D. B. Marrocco, Piedi monte, Napoli 1961.

D. B. Marrocco, Piedimonte Matese = D. B. Marrocco, Piedimonte Matese, Napoli 1980

R. Marrocco, Alife = R. Marrocco, Memorie storiche d'Alife, Piedimonte d'Alife (CE) 1926.

Marrou, Movolkós åvíp = H.-I. Marrou, Movolkós åvińp. Étude sur les scénes de la vie intellectuelle figurant sur les monuments funéraires romains (Bibl. de l'Inst. Franc. Naples s. $\left.1^{\circ}, 4\right)$, Grenoble 1938

Marruvium = M. A. AssetTA et AL., Da Marruvium a S. Benedetto dei Marsi. Storia - Archeologia - Antropologia Folklore (Comunità Montana "D" Valle del Giovenco Pescina), [Pescina s. a.] (liber machina dactylographica fortasse a. 1980 redactus)

Marsica I = G. Grossi - U. IRTI (Ed.), Carta Archeologica della Marsica (dalla Preistoria al Medioevo). In appendice: F. V. Colucci, Contributo per la ricostruzione del reticolo centuriale di Alba Fucens I, Avezzano (AQ) 2011.

MARTIN, Titulature épigraphique = A. MARTIN, La titulature épigraphique de Domitien (Beiträge zur klassischen Philologie 181), Frankfurt am Main 1987.
MartinOri, Via Salaria $=$ E. MArTinori, Via Salaria (antica e moderna). Via Claudia Nova. Studio storico-topografico, Roma 1931.

Masciotta, Molise = G. B. Masciotta, Il Molise dalle origini ai nostri giorni I-II, Napoli 1914/15.

Mascitti, Italia detecta = I. MascitTI, Italia detecta seu Corfinii monumenta plurimis ab Historicis excerpta opusculum (liber manu scriptus saec. XVII ex. nunc autem deperditus). Summarium f. 1-12 eiusdem operis servatur in bibliotheca publica Neapolitana olim signatum 344 nunc XV.D.4, cui titulus Antiqua monumenta Corfiniensium in Paelignis et circum positae regionis (etiam cf. MOMMSEN ad p. 297).

Massaro, Epigrafia metrica latina $=$ M. MAssaro, Epigrafia metrica latina di età repubblicana (Quaderni di "Invigilata lucernis” 1), Bari 1992.

Massimi, Amatrice $=$ A. Massimi, Amatrice e le sue ville . Notizie storiche, Amatrice (RI) [1958].

Mathieu, Aufidii = N. Mathieu, Histoire d'un nom. Les Aufidii dans la vie politique, économique et sociale du monde romain $I I^{e}$ siècle avant Jésus-Christ - III ${ }^{e}$ après Jésus-Christ, Rennes 1999.

MatTeini Chiari, Terventum = M. MatTeini ChIari, Ter ventum, in Ricognizione archeologica e documentazione cartografica (Quad. Ist. Top. Roma 6), Roma 1974, $143-$ 182.

Matтiocco, Castel di Sangro = E. Matтiocco, Castel di Sangro. Negli scritti dei geografi, storici, cronisti e viaggiatori dalle origini all'Unità d'Italia, L'Aquila 2017.

Matтiocco, Centri fortificati = E. Matтiocco, Centri fortificati vestini, Teramo 1986.

Matтiocco, Contributi = E. Matriocco, Contributi di archeologia peligna (Quad. Museo Sulmona 1), Sulmona (AQ) 1973.

Matтiocco, Il culto = E. Matтiocco, Il culto di Ercole tra $i$ Peligni (Quaderni di “Abruzzo" 8), Sulmona (AQ) 1973.

Matтiocco, Il Santuario = E. Matтiocco, Il Santuario di Ercole Curino presso Sulmona (Quad. Aquilano 9), Terni 1984.

Маттіоссо, Il territorio = E. Matтiocco, Il territorio superequano prima di Roma (Contr. Valle Peligna. Quaderno 2), Sulmona (AQ) 1983.

Matтiocco, Museo Aufidenate = E. Matтiocco, Il Museo Civico Aufidenate nel Convento della Maddalena in Castel di Sangro, Sulmona (AQ) 2005.

Matтiocco, Pietro Piccirilli = E. Matтiocco, Pietro Piccirilli. Una vita per l'arte e per la scuola, L'Aquila 2016.

Matтiocco, Protagonisti $=$ E. Matтiocco, Antonio De Nino e Vincenzo Balzano. Protagonisti della ricerca archeologica nel Sannio settentrionale, Lanciano (CH) 2001.

Matтiocco, Struttura urbana = E. MatтIocco, Struttura urbana e società della Sulmona medievale, Sulmona (AQ) 1978.

Mattiocco - De Panfilis, Pettorano = E. Mattiocco - E. De Panfilis, La terra di Pettorano. Documenti e scritti inediti, Teramo 1989.

MatTIOcCo - Orsini, Iscrizioni = E. MatTIOcCo - P. Orsini, Le iscrizioni della città di Sulmona dal XII al XVI secolo, L'Aquila 2015.

Mattiocco - Papponetti, Memoria e scrittura = E. MatTiocco - G. PApponetTi, Memoria e scrittura. Antonio De Nino (1833-1907). Mostra documentaria nell'80 della morte, Sulmona (AQ) 1987.

Mattiocco - Pettineldi, Sulmona nei secoli $=$ E. Mattiocco - P. Pettinelli, Sulmona nei secoli. Rassegna documentaria "Dalla capanna all'autostrada", Sulmona (AQ) 1981.

Maturanzio, $m s .=$ F. Maturanzio, Miscellanaea Umanistica (liber inter saec. XV ex. et saec. XVI in. manu scriptus 
in Biblioteca comunale Augusta adservatus Perugia sub n. C.61).

Maturo, Cascia = E. Maturo, Cascia, città di S. Rita, gemma dell'Umbria, Spoleto (PG) s. a. [post 1931].

MAURIzI, Cursus honorum = L. MAURIzI, Il cursus honorum senatorio da Augusto a Traiano. Sviluppi formali e stilistici nell'epigrafia latina e greca (Soc. Scient. Fennica, Comment. Human. Litt. 130), Helsinki 2013.

Mausoleum = H. von Hesberg - S. Panciera, Das Mausoleum des Augustus. Der Bau und seine Inschriften (Bayer. Akad. d. Wiss., Phil.-hist. Kl., Abh., N. F. 108), München 1994.

Maxfield, Decorations = V. A. Maxfield, The Military Decorations of the Roman Army, London 1981.

MAYer, Sprache = A. MAYer, Die Sprache der alten Illyrier I. Einleitung. Wörterbuch der illyrischen Sprachreste, Wien 1957.

Medicina e società = A. MARCONE (Ed.), Medicina e società nel mondo antico. Atti del Convegno di Udine, 4-5 ottobre 2005 (Studi Udinesi sul Mondo Antico 4), Firenze 2006.

Mediterraneo e la Storia $=$ L. Chioffi (Ed.), Il Mediterraneo e la Storia. Epigrafia e archeologia in Campania: letture storiche. Atti dell'incontro internazionale di studio, Napoli, 4-5 dicembre 2008, Napoli 2010.

Mélanges Jérôme Carcopino = Mélanges d'archeologie, d'épigraphie et d'histoire offerts à Jérôme Carcopino, Paris 1966.

Mélanges Jules Marouzeau = J. Ernst - C. J. Fordyce (Ed.), Mélanges de Philologie, de Littérature et d'Histoire anciennes offerts à J. Marouzeau par ses Collégues et Éléves Étrangers, Paris 1948.

Mélanges Marcel Le Glay = Y. Le Bohec (Ed.), L’Afrique, la Gaule, la religion à l'époque romaine. Mélanges à la mémoire de Marcel Le Glay (Coll. Latomus 226), Bruxelles 1994.

Mélanges Raymond Chevallier $=$ R. BEDON - P. M. MARTIN (Ed.), Mélanges offerts à Raymond Chevallier II. 2. Histoire et archéologie (Caesarodunum 29), Tours 1995.

Melchiori, Forum Novum = E. Melchiori, Storia e topografia dell'antico municipio romano di Forum Novum in Sabina e del suo territorio, Foligno (PG) 1904/05.

Mello, Mens Bona = M. Mello, Mens Bona. Ricerca sull'origine e sullo sviluppo del culto (Collana di Studi Greci 46), Napoli 1968.

Memoria poetica $=$ A. Pistellato (Ed.), Memoria poetica e poesia della memoria. La versificazione epigrafica dall'antichità all'umanesimo (Studi di archivistica, bibliografia e paleografia 3), Venezia 2014.

Memoriae civitatum = A. RuIz-GutiérREZ - C. CorTÉs-BÁrCENA (Ed.), Memoriae civitatum. Arqueología y Epigrafía de la ciudad romana. Estudios en homenaje a José Manuel Iglesias Gil, Santander 2017.

Memorie di Norcia = Liber manu scriptus saec. XVII-XVIII, cui titulus Memorie di Norcia insertus in fasciculo Zibaldone Fusconi in bibliotheca communali oppidi Norcia olim adservatus sine inv. n.; postquam terrae motus d. 24 m. Aug. et d. 30 m. Oct. a. 2016 universum oppidum diruerunt, liber Spoleto est translatus.

Menendez Arguin, Pretorianos = A. R. Menendez Arguin, Pretorianos: la guardia imperial de la antigua Roma, Madrid 2006.

Mennella - Apicella, Corporazioni professionali = G. MenNella - G. Apicella, Le corporazioni professionali nell'Italia romana: un aggiornamento al Waltzing (Quaderni del Dipartimento di scienze dell'antichità dell'Università degli Studi di Salerno 25), Napoli 2000.

Mennone, Riassunto storico = G. Mennone, Riassunto storico dell'antico Sannio, specialmente di Alife, Telese e Cerreto degli attuali paesi che n'ebbero origine e dei Signori che li dominarono fino all'abolizione feudale, [Piedimonte d'Alife (CE) 1894].

Mercati permanenti $=$ E. Lo CASCIO (Ed.), Mercati permanent e mercati periodici nel mondo romano. Atti degli Incontri capresi di storia dell'economia antica, Capri, 13-15 ottobre 1997 (Pragmateiai 2), Bari 2000.

Mercator placidissimus = F. CoARelli - H. Patterson (Ed.), Mercator placidissimus. The Tiber Valley in Antiquity. New research in the upper and middle river valley. Rome, 27-28 February 2004 (Quaderni di Eutopia 8), Roma 2008.

Merlat, Répertoire $=$ P. Merlat, Répertoire des inscriptions et monuments figurés du culte de Jupiter Dolichenus, Paris 1951.

Mertens, Alba Fucens = J. Mertens, Alba Fucens, Bruxelles 1981.

Méthode et pratiques = J. Desmulliez - CHr. HoËT-van CAUWENBERGHE (Ed.), Le monde romain à travers l'épigraphie: Méthodes et pratiques. Actes du XXIV Colloque international de Lille (8-10 novembre 2001) (Editions du Conseil Scientifique de l'Université Charles-de-Gaulle, Lille 3, Travaux et recherches), Villeneuve d'Asq 2005.

Metrische Inschriften = P. KRUSCHWITZ (Ed.), Die metrischen Inschriften der römischen Republik, Berlin - New York 2007.

Mezzadri, Memorie = B. Mezzadri, Memorie critiche istoriche della venerabile chiesa collegiata e parrocchiale di San Cesidio prete e martire nella terra di Trasacco vicina al lago di Fucino detto di Celano, di Marso, di Tagliacozzo, Roma 1769.

Michaeli, Memorie storiche I-IV = M. Michaeli, Memorie storiche della città di Rieti e dei paesi circostanti dall'origine all'anno 1560 I-IV. Ed. F. Gori - E. MercatantI, Rieti $1897 / 99$.

Migliario, Aspetti = E. Migliario, Uomini, terre e strade. Aspetti dell'Italia centroappenninica fra antichità e alto medioevo (Munera. Studi storici sulla Tarda Antichità 5), Bari 1995.

Migliorati, Iscrizioni = G. Migliorati, Iscrizioni per la ricostruzione storica dell'impero romano da Marco Aurelio a Commodo, Milano 2011.

Miscellanea = F. Rebecchi (Ed.), Miscellanea di studi archeologici e di antichità IV (Deputazione di Storia Patria per le antiche provincie modenesi. Biblioteca n. s. 134), Modena 1995.

Miscellanea Antonio Mario Radmilli = P. STODuti (Ed.), Miscellanea archeologica in onore di Antonio Mario Radmilli, Pisa 1994.

Miscellanea Emilio Marin = Hrv. G. JURIŠIĆ (Ed.), Zbornik u cast Emilia Marina za. 60. rođendan - Miscellanea Emilio Marin sexagenario dicata (Kačić. Zbornik Franjevačke provincije Presvetog Otkupitelia - Acta Provinciae SS. Redemptoris Ordinis Fratrum Minorum in Croatia 41/43, 2009/10), Split 2011.

Miscellanea Lidio Gasperini = G. PACI (Ed.), ЕПІГРАФАI. Miscellanea epigrafica in onore di Lidio Gasperini (Ichnia 5), Tivoli (RM) 2000.

Miscellanea Marc Mayer = A. Guzmán Almagro - J. Velaza (Ed.), Miscellanea philologica et epigraphica Marco Mayer oblata (Anuari de filologia antiqua et mediaevalia 8), Barcelona 2018.

Miscellanea Maristella Pandolfini = E. Benelli (Ed.), Per Maristella Pandolfini. cên zic zixuxe (Mediterranea. Supplemento 10), Pisa 2014.

Misurare il tempo = M. G. Angeli Bertinelli - A. Donati (Ed.), Misurare il tempo. Misurare lo spazio. Atti del Colloquio AIEGL - Borghesi 2005, Bertinoro, 20-22 ottobre 2005 (Epigrafia e Antichità 25), Bologna - Faenza 2006. 
Mócsy, Nomenclator = A. Mócsy et AL., Nomenclator provinciarum Europae Latinarum et Galliae Cisalpinae cum indice inverso (Diss. Pann. III 1), Budapest 1983.

Modalità insediative $=\mathrm{E}$. Lo CASCIO $-\mathrm{A}$. STORCHI Marino (Ed.), Modalità insediative e strutture agrarie nell'Italia meridionale in età romana (Pragmateiai 7), Bari 2001.

Modelli epistemologici $=$ P. Molinelli - I. Putzu (Ed.), Modelli epistemologici, metodologie della ricerca e qualità del dato. Dalla linguistica storica alla sociolinguistica storica (Metodi e prospettive 14), Milano 2015.

Molise $=$ N. Paone (Ed.), Il Molise. Arte, cultura, paesaggi, Roma 1990.

Molise. Archeologia = A. Ceccarelli - G. Fratianni (Ed.), Molise (Archeologia delle Regioni d'Italia), Roma 2017.

Molise. Esperienze = G. De Benedittis (Ed.), Molise. Esperienze di Survey. Riccia - Oratino - Castropignano, Isernia 2008.

Molise medievale = C. Ebanista - A. Monciatti (Ed.), Il Molise medievale. Archeologia e arte, Borgo S. Lorenzo (FI) 2010.

Molle, Aquinum = C. Molle, Le fonti letterarie antiche su Aquinum e le epigrafi delle raccolte comunali di Aquino (Ager Aquinas. Storia e archeologia nella media valle dell'antico Liri 5), Aquino (FR) 2011.

Mommsen, Gesammelte Schriften $=$ Tн. Mommsen, Gesammelte Schriften I-VIII, Berlin 1905/13.

Mommsen, Inscriptiones regni Neapolitani $=$ Tн. Mommsen, Inscriptiones regni Neapolitani Latinae, Lipsiae 1852.

Mommsen, Staatsrecht ${ }^{3}$ I-III $=$ Th. Mommsen, Römisches Staatsrecht ${ }^{3}$ I-III, Leipzig 1887/88 (lucis ope iterum impressum Basel - Stuttgart 1963).

Monaco, Pettorano sul Gizio = P. Monaco, Pettorano sul Gizio nella corona radiosa dei Cantelmo, Sulmona (AQ) 1980.

Montagna, Topografia $=$ C. Montagna, Topografia archeologica della via Ternana da Passo Corese a Torri in Sabina (thesis a. 1962 machina dactylographica redacta doctoris dignitatis nominisque inter schedas a GiUsepPe LugLi collectas apud archivum instituti antiquae descriptionis locorum, quod Italice Topografia Antica dicitur, sedis litterarum urbis Romae, cui Athenaeo nomen olim " $L a$ Sapienza" hodie "Sapienza" est impositum, servata).

Montebuono = M. VAlenti (Ed.), Montebuono e il suo territorio. Storia, architettura e restauri. Inizia la ricerca, Roma 2007.

Monteleone Sabino = D. Monaco - V. Tomassetti - R. Tomassetti, Monteleone Sabino. Storia di un'antica città, una santa, un borgo medioevale, Roma 1984

Monteleone Sabino già Trebula Mutuesca = D. Monaco V. Tomassetti - R. Tomassetti - G. CApitani, Monte leone Sabino già Trebula Mutuesca. Storia di un'antica città, di un borgo, di una santa (Contributi: G. Alvino - P. L. Cecca - M. Mayer y Olivé - V. Fiocchi Nicolai S. Rossi - F. Tron), Terni 2009

Monteluco e i monti sacri $=$ Monteluco e $i$ monti sacri. Atti dell'incontro di studio, Spoleto, 30 settembre - 2 ottobre 1993 (CISAM. Miscellanea 9), Spoleto (PG) 1994.

Monti Lucretili = G. De Angelis (Ed.), Monti Lucretili. Parco regionale naturale ${ }^{5}$, Tivoli (RM) 1995.

Monumenta et memoria = J. M. IGLESIAS GIL - A. RuIZGutiérrez (Ed.), Monumenta et memoria. Estudios de epigrafía romana, Roma 2017.

Mora, Prosopographia Isiaca $=$ F. Mora, Prosopographia Isiaca (EPRO 113), Leiden 1990.

Morandi, Epigrafia italica I = A. Morand, Epigrafia italica (Bibl. Arch. 2), Roma 1982

Morandi, Epigrafia italica II = A. Morand, Epigrafia italica 2 (Bibl. Arch. 57), Roma 2017.
Morandi, Iscrizioni medio-adriatiche = A. MORANDI, Le iscrizioni medio-adriatiche (Biblioteca di "Studi Etruschi" 7), Firenze 1974

A. Morelli, Norcia $=$ A. Morelli, Guida di Norcia, Norcia (PG) 1958.

M. Morelli, Collepietro = M. Morelli, Collepietro: storia, documenti, tavole ricostruttive I, Chieti Scalo 1991.

L. Moretti, Scritti scelti = L. Moretti, Tra epigrafia e storia. Scritti scelti e annotati (Vetera 5), Roma 1990.

M. Moretti, Museo nazionale = M. Moretti, Museo Nazionale d'Abruzzo nel castello cinquecentesco dell'Aquila, L'Aquila 1968.

Morico, Castelvecchio Calvisio = W. Morico, Castelvecchio Calvisio. Storia e territorio, L'Aquila 2003.

Morini, ms. = A. Morini, Carte Morini (liber priore parte saec. XX manu exaratus in monasterio sororum Augustinianarum S. Ritae in oppido Cascia servatus sine inv. n.).

Morini, Cascia $=$ A. Morini, Cascia nella natura, nella storia, nell'arte, Perugia 1913

Morini, Cursula $=$ A. Morini, Cursula, Roma 1896

Morini, Scoperta ${ }^{2}=$ A. Morini, La scoperta di una tomba antichissima a Monteleone presso Cascia ${ }^{2}$, Perugia 1904.

Moussy, Gratia et sa famille $=$ C. Moussy, Gratia et sa famille (Publications de la Faculté des Lettres et Sciences humaines de l'Université de Clermont-Ferrand 25), Paris 1966.

Mrozer, Distributions = S. Mrozek, Les distributions d'argent et de nourriture dans les villes italiennes du HautEmpire romain (Coll. Latomus 198), Bruxelles 1987.

MrozeK, Prix et rémunération $=\mathrm{S}$. MrozeK, Prix et rémunération dans l'Occident romain (31 av.n. è. -250 de n.è.) (Wydziat I Nauk Społecznych i humanistycznych. Seria monografii 55), Gdańsk 1975.

Mrozek, Recueil d'études = S. Mrozer, Argent, société et épigraphie romaine $\left(1^{e r}-3^{e}\right.$ siècles $)$. Recueil d'études complétées (Collection Moneta 38), Wetteren 2004.

MuccI, $m s .=$ L. MuccI, Libri manu scripti a. 1880/84 servati Romae apud Archivio Centrale dello Stato (EUR). MPI. Dir. Gen. AABBAA. 1860-1890, vol. I, Tomo II, b. 14, fasc. 24, sf. 7 .

Municipal Structures = F. Russo (Ed.), Municipal Structures in Roman Spain and Roman Italy. A Comparison. Proceedings of the Colloquium Vienna, 3rd July 2018 (Wiener Beiträge zur Alten Geschichte online 3), Wien 2020.

Munus Laetitiae = F. Camia - L. Del Monaco - M. Nocita (Ed.), Munus Laetitiae. Studi miscellanei offerti a Maria Letizia Lazzarini I-II (Studi e ricerche 70), Roma 2018.

Musei Capitolini = S. PANCIERA (Ed.), La collezione epigrafica dei Musei Capitolini. Inediti - Revisioni - Contributi al riordino (Tituli 6), Roma 1987.

Museo Civico Archeologico "F. Savini" - Teramo = P. DE Felice - V. Torrieri (Ed.), Museo Civico Archeologico "F. Savini" - Teramo, Teramo 2006.

Museo Civico di Sulmona = A. Colangelo - E. Mattiocco - R. Tuteri, Guida alla visita del Museo Civico di Sulmona, Viterbo 2000

Museo Comunale di Vasto = E. Fabbricotti (Ed.), Il Museo Comunale di Vasto. Catalogo della collezione archeologica, Chieti 1984.

Museo Emidiano = Agnone. Il Museo Emidiano. Il territorio. Catalogo della Mostra organizzata dall'Archeoclub "F.S. Cremonese”. Chiesa di S. Chiara di Agnone, 10-31 agosto 1985, Matrice (CB) 1985

Museo Palazzo Santi = G. Gentilini - M. Matteini Chiari (Ed.), Museo di Palazzo Santi. Chiesa di S. Antonio Abate. Circuito Museale di Cascia (Catalogo Regionale dei Beni Culturali dell'Umbria 77), Perugia 2013.

Museo Sannitico = A. Di Niro (Ed.), Il Museo Sannitico di Campobasso. Catalogo della Collezione Provinciale, Pescara 2007 
Muzzioli, Cures = M. P. Muzzioli, Cures Sabini (Forma Italiae. Regio IV. Vol. II), Firenze 1980.

Nacimiento = F. Beltrán Lloris - B. Díaz Ariño (Ed.), El nacimiento de las culturas epigráficas en el Occidente mediterráneo. Modelos romanos y desarrollos locales (III-I a. E.) (Anejos de AEspA 85), Madrid 2018.

NARDI, ms. = E. NARDI, Ruderi di Ville Romano-Sabine nei dintorni di Poggio Mirteto illustrati dal Dott.re Prof.re Cav.re Ercole Nardi R.o Ispettore degli Scavi e Monumenti. Anno 1885 (liber manu scriptus ex viginti duobus fasciculis compositus, scil. n. I-XXII, hodie servatus Romae apud Biblioteca di Archeologia e Storia dell'Arte (BiASA), sub Manoscritti e Fondi Archivistici, sign. n. 96 [cf. D. SCARPaTI, in Lazio \& Sabina VII 141-143; EUnD., Ruderi delle ville romano sabine nei dintorni di Pogrio Mirteto illustrati dal Prof. Ercole Nardi, 1885, Roma 2010]; exemplum alterum a G. CicCONETTI a. 1928 descriptum servatur Poggio Mirteto apud dominum Gualtiero Consumati; exemplum tertium, fasciculis autem n. XXIV carens, invenies inter schedas a GiUseppe LugLi collectas nunc apud archivum instituti antiquae descriptionis locorum, quod Italice Topografia Antica dicitur, sedis litterarum urbis Romae, cui Athenaeo nomen olim " $\mathrm{La}$ Sapienza" hodie "Sapienza" est impositum, servatum).

M. Nassa, Catalogo = M. Nassa, Catalogo del Museo Alifano I. Oggetti di antichità (Introduzione, indici e note) (Associazione Storica del Medio Volturno. Quaderno 23 [n. s. 6]), Piedimonte Matese (CE) 1995 (agitur de editione libelli machina dactylographica compositi a. 1935 a RAFFAELE Marrocco in bibliotheca municipali vici Alife sine inv. $\mathrm{n}$. adservati).

V. NASSA, Elaborazioni = V. NAsSA, Elaborazioni e aggiunte fotografiche ad alcuni scritti di Mario Nassa II, Raviscanina (CE) 2011.

Natale, Fossacesia = G. Natale, Fossacesia. Origine e storia del nome. In occasione dei 150 anni dell'Unità d'Italia e del toponimo moderno (1861-2011), Fossacesia (CH) 2011.

Navarro Caballero, Perfectissima femina $=$ M. Navarro Caballero, Perfectissima femina. Femmes de l'élite dans l'Hispanie romaine I-II, Bordeaux 2017.

Necropoli di Fossa I-IV = La necropoli di Fossa I. Le testimonianze più antiche, ed. S. Cosentino - V. D'ERCole G. Mieli, Pescara 2001. II. I corredi orientalizzanti e arcaici, ed. V. D’ERcole - E. Benelli, Pescara 2004. IV. L'età ellenistico-romana, ed. V. D'ERCOLE - M. R. COPERsino, Pescara 2003.

Nessi - Ceccaroni, Spoleto $=$ S. Nessi - S. Ceccaroni, $D a$ Spoleto a Monteleone attraverso il Monte Coscerno (Itinerari spoletini 1), Spoleto (PG) 1972.

Newton, Epigraphical Evidence = H. C. Newton, The Epigraphical Evidence for the Reigns of Vespasian and Titus (Cornell Studies in Classical Philology 16), Ithaca (N.Y.) 1901.

NiCOLET, L'ordre équestre I-II = C. NICOLET, L'ordre équestre à l'époque républicaine (312-43 av. J.-C.) I-II (Bibl. des Écoles Fr. d'Athènes et de Rome 207), Paris 1966/74.

Nissen, Italische Landeskunde $=$ H. Nissen, Italische Landeskunde II. Die Städte, Berlin 1902.

Noctes Campanae = W. V. Harris - E. Lo CAScio (Ed.), Noctes Campanae. Studi di storia antica ed archeologia dell'Italia preromana e romana in memoria di Martin W. Frederiksen. Atti del Convegno Internazionale tenutosi a Capri nei giorni 8-10 ottobre del 2000 (Itala Tellus 1), Napoli 2005.

NüNNERICH-Asmus, Basilika und Portikus = A. NüNNERICHAsmus, Basilika und Portikus. Die Architektur der Säulenhallen als Ausdruck gewandelter Urbanität in später Republik und früher Kaiserzeit, Köln 1994.
Nuove ricerche $=$ L. GASPERINI - G. PACI (Ed.), Nuove ricerche sul culto imperiale in Italia. Atti dell'incontro di studio, Ancona, 31 gennaio 2004 (Ichnia 7), Tivoli (RM) 2008.

Nursia e l'ager Nursinus = S. SISANI (Ed.), Nursia e l'ager Nursinus. Un distretto sabino dalla praefectura al municipium, Roma 2013.

Öffentlichkeit = W. EcK - P. FunKE et AL. (Ed.), Öffentlichkeit - Monument - Text. XIV Congressus Internationalis Epigraphiae Graecae et Latinae 27.-31. Augusti MMXII Akten (CIL Auctarium. Series nova 4), Berlin 2014.

Officina epigrafica = A. DONATI - G. POMA (Ed.), L'officina epigrafica romana in ricordo di Giancarlo Susini. Atti del Colloquio AIEGL - Borghesi 2003, Bertinoro, 16-18 settembre 2010 (Epigrafia e Antichità 30), Bologna - Faenza 2012.

Окоќ, Album senatorum $\mathrm{I}-\mathrm{II}=\mathrm{D}$. ОкоŃ, Album senatorum . I. Senatores ab Septimii Severi aetate usque ad Alexandrum Severum (193-235 AD); Album senatorum. II. Senatorowie epoki Sewerów (193-235 r. n. e.). Studium prozopograficzne (Szczecińkie Studia nad Starożynościà 5), Szczecin 2017.

Omagiu Constantin Daicoviciu = Omagiu lui Constantin Daicoviciu cu prilejul implinirii a 60 de ani, Bucuresti 1960.

Onomastica $=$ P. PoccetTI (Ed.), L'onomastica dell'Italia antica. Aspetti linguistici, storici, culturali, tipologici e classificatori. Atti del convegno, Roma, il 13-16 novembre 2002 (Coll. de l'École Fr. de Rome 413), Paris - Rome 2009.

Opinione pubblica $=$ M. G. ANGeLI BerTineLli - A. DonATI (Ed.), Opinione pubblica e forme di comunicazione a Roma: Il linguaggio dell'epigrafia. Atti del Colloquio AIEGL - Borghesi 2007, Bertinoro, 21-23 giugno 2007 (Epigrafia e Antichità 27), Bologna - Faenza 2009.

Orelli, Collectio = J. [= G.] G. Orelli, Inscriptionum Latinarum selectarum amplissima collectio ad illustrandam Romanae antiquitatis disciplinam accomodata ac magnarum collectionum supplementa complura emendationesque exhibens I-II, Turici 1828. III. Collectionis Orellianae supplementa emendationesque exhibens, ed. G. [= W.] HeNZEN, Turici 1856.

Oricola $=$ S. Lapenna (Ed.), Oricola. Dalle cittadelle degli Equi alla Carsioli romana, Sulmona (AQ) 2003.

L. Orlandi, I Marsi = L. Orlandi, I Marsi e l'origine di Avezzano, Napoli 1967.

S. Orlandi, Epigrafia anfiteatrale $=$ S. OrLandi, Epigrafia anfiteatrale dell'Occidente Romano VI. Roma. Anfiteatri e strutture annesse con una nuova edizione e commento delle iscrizioni del Colosseo (Vetera 15), Roma 2004.

Orlandi - Veri, Museo Lapidario = L. T. Orlandi - E. Veri, Museo Lapidario di Avezzano, Avezzano (AQ) 1989.

Ortiz de Zárate, Nutrices = S. C. Ortiz de ZÁrate, Nutrices en el imperio Romano. I. Estudio de las fuentes y prosopografía, Valladolid 2005. II. Estudio social, Valladolid 2006.

Ortona Italica $=$ Ortona Italica $($ Associazione Ortonese di Storia Patria), Ortona (CH) 1998.

Ottavi, Ferrovia Roma-Sulmona = V. OtTAvi, La ferrovia Roma-Sulmona e la Marsica, Avezzano (AQ) 1888.

OtTaviani, San Vittorino = V. OtTaviani, Il cimitero cristiano antico e la chiesa di San Vittorino presso Amiterno (Studi e ricerche 1), L'Aquila 1987.

Ovidio $=$ P. Fedeli - G. Rosati (Ed.), Ovidio 2017. Prospettive per il terzo millennio. Atti del Convegno Internazionale, Sulmona, 3-6 aprile 2017, Teramo 2018.

OXÉ- COMFort, CVA = A. OXÉ- H. COMFort, Corpus vasorum Arretinorum. A catalogue of the signatures, shapes and chronology of Italian sigillata (Antiquitas 3), Bonn 1968. 
Pace - Scarascia, Antiche civiltà = D. PaCe - R. Scarascia, Antiche civiltà peligne, Sulmona (AQ) 1977.

PACI, Ricerche = G. PACI, Ricerche di storia e di epigrafia romana delle Marche (Ichnia 11), Tivoli (RM) 2008.

PACI, Senatori e cavalieri = G. PACI, Senatori e cavalieri romani nelle iscrizioni di Forum Clodii (Quaderni della Forum Clodii 4), Bracciano (VT) 1977.

Paesaggi I = G. VolPe - M. TurChiano (Ed.), Paesaggi e insediamenti urbani in Italia meridionale fra Tardoantico $e$ Altomedioevo. Atti del Primo Seminario sul Tardoantico e l'Altomedioevo in Italia Meridionale, Foggia, 12-14 febbraio 2004 (Insulae Diomedeae 4), Bari 2005.

Paesaggi II = G. VolPe - R. Giuliani (Ed.), Paesaggi e insediamenti urbani in Italia meridionale fra Tardoantico e Altomedioevo. Atti del Secondo Seminario sul Tardoantico e l'Altomedioevo in Italia Meridionale, Foggia - Monte Sant'Angelo, 27-28 maggio 2006 (Insulae Diomedeae 14), Bari 2010.

Paesaggi costieri = Atti della Tavola Rotonda AdriAtlas e i paesaggi costieri dell'Abruzzo tra antichità e altomedioevo. Bari, 22-23 maggio 2017, Bari 2019.

Paesaggi rurali $=$ G. Volpe (Ed.), Storia e archeologia globale dei paesaggi rurali in Italia fra Tardoantico e Medioevo (Insulae Diomedeae 34), Bari 2018.

Pagani, Avezzano = G. Pagani, Avezzano e la sua storia, Casamari (FR) 1968.

PAIS = Corporis Inscriptionum Latinarum supplementa Italica I. Additamenta ad vol. V Galliae Cisalpinae, ed. H. [= E.] Pais (Mem. Accad. Lincei 4, 5), Romae 1888.

Palma, $m s .=$ T. PALma, Compendio istorico dell'antichissima terra del Vasto in Abbruzzo citra nel Regno di Napoli del signor Tommaso Palma Segretario dell'eccellentissima Casa del Vasto (liber a. 1690 manu scriptus in bibliotheca communali Vastensi sine inv. n. servatus).

Palmegiani, Rieti $=$ F. Palmegiani, Rieti e la regione sabina . Storia, arte, vita, usi e costumi del secolare popolo sabino, Roma 1932 (lucis ope iterum impressum Rieti 1988).

Palozzi, Villavallelonga $=$ L. PalozzI, Storia di Villavallelonga. Centro del Parco Nazionale d'Abruzzo e rocca medioevale dei Marsi (Centro di Ricerche Letterarie Abruzzesi dell'Università degli Studi dell'Aquila. Studi e testi 8), Roma 1982.

Panciera, Epigrafi $=$ S. PANCIERA, Epigrafi, epigrafia, epigrafisti. Scritti vari e inediti (1956-2005) con note complementari e indici I-III (Vetera 16), Roma 2006.

Pane, Carta archeologica = C. PAne, Carta Archeologica della Valle del Sagittario (liber machina dactylographica redactus thesis doctoris dignitatis nominisque a. 2010/11 Romae impetratae in Università degli Studi di Roma "La Sapienza”).

Pani ERmini, Il santuario = L. PANi ERmini, Il santuario del martire Vittorino in Amiternum e la sua Catacomba (Quad. Aquilano 1), Terni 1975.

Pantoni, Le chiese $=$ A. Pantoni, Le chiese e gli edifici del Monastero di San Vincenzo al Volturno (Miscellanea cassinese 40), Montecassino (FR) 1980

PAPE, Griechische Kunstwerke = M. PAPE, Griechische Kunstwerke aus Kriegsbeute und ibre öffentliche Aufstellung in Rom von der Eroberung von Syracus bis in augusteische Zeit, Hamburg 1975.

Papers in Italian Archaeology = C. MaLone - S. STOddarT (Ed.), Papers in Italian Archaeology. The Cambridge Conference [third Conference of Italian Archaeology, Cambridge 1984] 4. 4. Classical and medieval archaeology (BAR. International Series 246), Oxford 1985.

Parco Nazionale d'Abruzzo = Il territorio del Parco Nazionale d'Abruzzo nell'antichità. Atti del I Convegno nazionale di archeologia, Villetta Barrea, 1-3 maggio 1987, Civitella Alfedena (AQ) 1988.
Parenté et stratégies = J. Andreau - H. Bruhns (Ed.), Parenté et stratégies familiales dans l'antiquité romaine. Actes de la table ronde des 2-4 octobre 1986, Paris, Maison de sciences de l'homme (Coll. de l'École Fr. de Rome 129), Rome 1990.

Paribeni, Terme di Diocleziano ${ }^{2}=$ R. PARIBeni, Le Terme di Diocleziano e il Museo Nazionale Romano ${ }^{2}$, Roma 1932.

PARIS, ms. I = V. PARIs, Iscrizioni di Norcia nel Corpus Inscriptionum Latinarum (liber saec. XX in. manu exaratus in bibliotheca communali oppidi Norcia olim adservatus, Fondo Reguardati sine inv. n.; postquam terrae motus d. 24 m. Aug. et d. 30 m. Oct. a. 2016 universum oppidum diruerunt, Spoleto est translatus).

PARIs, $m s$. II = V. PARIS, Di alcuni antichi residui della città di Norcia (liber a. 1906 ut videtur manu scriptus in bibliotheca communali oppidi Norcia olim adservatus, Fondo Reguardati sine inv. n.; postquam terrae motus d. 24 m. Aug. et d. 30 m. Oct. a. 2016 universum oppidum diruerunt, Spoleto est translatus).

PARIS, $m s$. III = V. PARIs, Schede di epigrafi inursine e altre carte (liber a. 1906/11 ut videtur manu scriptus in bibliotheca communali oppidi Norcia olim adservatus, Fondo Reguardati sine inv. n.; postquam terrae motus d. $24 \mathrm{~m}$. Aug. et d. 30 m. Oct. a. 2016 universum oppidum diruerunt, Spoleto est translatus).

PArIs, Norcia = V. Paris, Guida di Norcia, Spoleto (PG) 1906. Parisi, Alife $=$ G. Parisi, Alife e le sue chiese. Itinerario storico-artistico alle radici del sacro, Piedimonte Matese (CE) 2006.

PASSERINI, Coorti pretorie = A. PASSERINI, Le coorti pretorie (Stud. Ist. It. Stor. Ant. 1), Roma 1939.

Patrizi-Forti, Memorie = F. Patrizi-Forti, Delle memorie storiche di Norcia, Norcia (PG) 1869/74 (inde Bologna $1968=1987)$.

Patterson, Landscapes and Cities = J. R. Patterson, Land scapes and Cities. Rural Settlement and Civic Transformation in Early Imperial Italy, Oxford (NY) 2006.

Peachin, Titulature $=$ M. Peachin, Roman Imperial Titula ture and Chronology, A. D. 235-284 (Studia Amstelodamensia ad epigraphicam, ius antiquum et papyrologicam pertinentia 29), Amsterdam 1990.

PeKÁry, Untersuchungen $=$ TH. PeKÁRY, Untersuchungen $z u$ den römischen Reichsstrassen (Antiquitas 1. 17), Bonn 1968.

Peltuinum = A. Campanelli (Ed.), Peltuinum. Antica città sul tratturo, Brescia - Pescara 1996.

Penne $=$ Penne e l'area vestina nel Bullettino di Storia Patria Scritti di I. LudovisI - C. Rivera - G. De CAESARIS (1897, 1925, 1927, 1929-1930), L'Aquila 1991.

Peoples = G. D. Farney - G. Bradley (Ed.), The Peoples of Ancient Italy, Berlin - Boston 2017.

Per Renato Badalì = In amicitia per Renato Badalì. Una giornata di studi (Viterbo, lunedì 8 giugno 2015), Viterbo 2015.

Pere Miquel Carbonell = A. Guzmán Almagro - X. Espluga - M. Ahn (Ed.), Pere Miquel Carbonell i el seu temps (1434-1517), Barcelona 2016.

Perea Yébenes, Collegia militaria $=$ S. Perea Yébenes, Collegia militaria. Asociaciones militares en el Imperio romano (Signifer 3), Madrid 1999 (lucis ope iterum impressum Madrid 2013).

Peregrinationes = G. GonZÁlez Germain (Ed.), Peregrinationes ad inscriptiones colligendas. Estudios sobre epigrafía de tradición manuscrita (Congressos 14), Bellaterra 2016.

Perin, Lexicon I-II = I. Perin, Lexicon totius Latinitatis V-VI. Onomasticon I-II, Patavii 1940.

Perrotta, Vicus Anninus = E. Perrotta, Per la storia del Vicus Anninus nel territorio di Marruvium (liber machina dactylographica redactus thesis doctoris dignitatis nominis- 
que a. 1995/96 Romae impetratae apud Università degli Studi di "Roma 3". Facoltà di Lettere e Filosofia).

Perrotti, Note storiche = M. Perrotti, Note storiche su Piedimonte d'Alife e limitrofi, Piedimonte d'Alife (CE) 1896.

Persichetti, $m s .=$ N. Persichetti, Libri manu scripti saec. XIX ex. - XX in. servati Romae apud Archivio Centrale dello Stato. MPI. Dir. Gen. AABBAA. Antichità e Scavi. II versamento.

Persichetti, Via Salaria $=$ N. Persichetti, La Via Salaria ne circondarii di Roma e Rieti, Roma 1910.

Persichetti, Viaggio archeologico = N. Persichetti, Viaggio archeologico sulla Salaria nel circondario di Cittaducale con appendice sulle antichità dei dintorni e tavola topografica, Roma 1893.

Personal Names $=$ T. MeIssner (Ed.), Personal Names in the Western Roman World. Proceedings of a Workshop held at Pembroke College, Cambridge, 16-18 Septembre 2011, Berlin 2012.

Petraccia Lucernoni, Questori municipali $=$ M. F. PetracCIA LuCERNONI, I questori municipali dell'Italia antica (Stud. Ist. It. Stor. Ant. 41), Roma 1988.

Petraccia Lucernoni, Stationarii $=$ M. F. Petraccia LucerNONI, Gli stationarii in età imperiale (Serta antiqua 3), Roma 2001.

Petrevcic, Marruvium = M. Petrevcic, Marruvium capitale dei Marsi (thesis doctoris dignitatis nominisque a. 1970/71 in Universitate Teatina machina dactylographica redacta).

Petrini - Cordella, Guida $=$ S. Petrini - R. Cordella, Guida di Norcia e del suo territorio, Norcia (PG) 1978.

Petrovitsch, Legio = H. Petrovitsch, Legio II Italica (Forschungen in Lauriacum 13), Linz 2006.

Petrucci, $m s .=$ L. I. [= G.] Petrucci, Storia di Telese (liber inter a. 1853/63 f. 411 fortasse exaratus nunc servatus Neapoli una cum dissertationibus L'idrologia delle acque telesine et Statuti di Cerreto ab ipso Libero Iosepho Petrucci (1793-1865) compositis in bibliotheca sodalicii Società Napoletana di Storia Patria, sign. ms. XXXIII-A-6. Recensionem alteram eiusdem operis excussisse in usum Theodor Mommsen videtur Friedrich von Duhn, de qua recensione vide p. $205 \mathrm{n}$. V; nunc etiam vide A. Cutillo et AL. (Ed.), Idrologia delle minerali telesine di Libero Petrucci, dottore in Medicina (1856), Telese Terme (BN), 2016).

Pfeiffer - Ashby, Carsioli = G. J. Pfeiffer - Th. Ashby, Carsioli. A Description of the Site and the Roman Remains, with Historical Notes and a Bibliography, Supplementary Papers of the American School of Classical Studies in Rome 1, Rome 1905, 108-140.

Pflaum, Carrières I-IV = H.-G. Pflaum, Les carrières procuratoriennes équestres sous le Haut-Empire romain I-IV (Institut Français d'Archéologie du Proche-Orient Beyrouth. Bibliothèque archéologique et historique 57, 14), Paris 1960/61.

Pflaum, Carrières Suppl. $=$ H.-G. Pflaum, Les carrières procuratoriennes équestres sous le Haut-Empire romain. Supplément (Institut Français d'Archéologie du ProcheOrient Beyrouth - Damas - Amman. Bibliothèque archéologique et historique 112), Paris 1982.

Pflaum, Procurateurs $=$ H.-G. Pflaum, Les procurateurs équestres sous le Haut-Empire romain, Paris 1950.

Pflaum, Scripta varia $\mathrm{I}=$ H.-G. Pflaum, Scripta varia $\mathrm{I}$. L'Afrique romaine, Paris 1978.

Pflaum, Scripta varia II = H.-G. Pflaum, Scripta varia II. La Gaule et l'Empire romain, Paris 1981.

Pflaum historien $=\mathrm{S}$. Demougin $-\mathrm{X}$. Lariot $-\mathrm{P}$. Cosme $-\mathrm{S}$. Lefebvre (Ed.), H.-G. Pflaum. Un historien $d u X X^{e}$ siècle (Bibl. de l'École Haut. Ét. Sc. Hist. III. 37), Genève 2006.

G. Piccirilli, Sulmona = G. Piccirilli, Sulmona. Guida storico-artistica, Sulmona (AQ) 1932.
P. PICCIRILli, Monumenti $=$ P. PICCIRILli, Monumenti architettonici sulmonesi descritti e illustrati (dal XIV al XV secolo), Lanciano (CH) 1888.

Pietilä-Castrén, Magnificentia publica $=$ L. PietiläCastrén, Magnificentia publica. The Victory Monuments of the Roman Generals in the Era of the Punic Wars (Soc. Scient. Fennica, Comment. Human. Litt. 84), Helsinki 1987.

Pietrangeli, Sabina $=$ C. Pietrangeli, La Sabina nell'antichità, in Rieti e il suo territorio, Milano 1976, 9-164.

Pignora amicitiae = M. CASSIA - C. Giuffrida - C. Molè A. PInzone (Ed.), Pignora amicitiae. Scritti di storia antica e storiografia offerti a Mario Mazza I-III (Storia e politica 9), Acireale (CT) 2012

Pikhaus, Levensbeschouwing = D. PikHaus, Levensbeschouwing en milieu in de latijnse metrische inscripties, Brussel 1978.

Pinna Vestinorum I = L. Franchi dell'Orto (Ed.), Pinna Vestinorum e il popolo dei Vestini (Storia e civiltà di Penne 1), Roma 2010.

Pinna Vestinorum II = L. Franchi Dell'Orto (Ed.), Pinna Vestinorum e la città romana (Storia e civiltà di Penne 2), Roma 2010.

PIR ${ }^{1}$ III = P. von Rohden - H. Dessau, Prosopographia Imperii Romani saec. I. II. III. Pars III, Berolini 1898 [auctorem laudavi, si feci, Dessau, qui opus a DE RoHDEN relictum supplevit terminavit edidit].

$P I R^{2}=$ E. Groag - A. Stein - L. Petersen et Al., Prosopographia Imperii Romani saec. I. II. III, ed. altera, I-VIII, Berolini 1933/2015.

PIRrI, Abbazia = P. PIrRI, L'abbazia di S. Eutizio in Valcastoriana presso Norcia e le chiese dipendenti, Castelplanio (AN) 1912 (ed. altera Romae 1960).

Pisani, Lingue dell'Italia $=$ V. PISANI, Manuale storico della lingua latina IV. Le lingue dell'Italia antica oltre il latino, Torino 1953.

Pisani, Testi latini arcaici ${ }^{2}=$ V. PIsAnI, Manuale storico della lingua latina III. Testi latini arcaici e volgari con commento glottologico ${ }^{2}$, Torino 1960

PIso, Fasti I-II = I. PIso, Fasti provinciae Daciae I. Die senatorischen Amtsträger. II. Die ritterlichen Amtsträger (Antiquitas 1. 43, 60), Bonn 1993/2013.

Pistellato, Memoria $=$ A. Pistellato, Stirpem nobilitavit honor. La memoria dei Senzi Saturnini tra retorica e storiografia (Supplementi di Lexis n. s. 2), Amsterdam 2015.

Pitoni - Salvi, Antrosano = G. Pitoni - A. Salvi, Antrosano: memoria e storia, Avezzano (AQ) 2000.

PIVA, Santuari $=$ V. PIVA, I santuari extraurbani del Sannio preromano e romano. Aspetti storici e archeologici (liber machina dactylographica redactus thesis doctoris dignitatis nominisque a. 2017/18 Romae impetratae apud "Sapienza" Università di Roma).

PlaCidi, Peltuinum = V. Placidi, La città e il territorio di Peltuinum nei Vestini: problemi dell'insediamento, Roma [1983].

Planta, Grammatik = R. von Planta, Grammatik der oskisch-umbrischen Dialekte, Straßburg 1897.

PLRE I-III = A. H. M. Jones - J. R. MarTINDALE - J. MorRIs, The Prosopography of the Later Roman Empire I. A. D. 260-395, Cambridge 1971. J. R. Martindale, The Prosopography of the Later Roman Empire II. A. D. 395527, Cambridge cet. 1980. ID., The Prosopography of the Later Roman Empire III A-B. A. D. 527-641, Cambridge 1992.

Pluralidad e integración = F. J. Navarro (Ed.), Pluralidad e integración en el mundo romano. Actas del Coloquio Internacional Italia Iberia - Iberia Italia, Pamplona-Olite del 15 al 17 de octubre de 2008 (Colección Mundo Antiguo n. s. 13), Pamplona 2010 
PME I-VI = H. Devijver, Prosopographia militiarum equestrium quae fuerunt ab Augusto ad Gallienum I-VI (Symbolae Facultatis litterarum et philosophiae Lovaniensis, Ser. A 3. 1-6), Leuven 1976/2001.

Poccetti, L'area superequana $=$ P. Poсcetti, L'area superequana nel quadro della documentazione dialettale peligna (Contr. Valle Peligna. Quaderno 1), Castelvecchio Subequo (AQ) 1982.

Poccetti, Nuovi documenti $=$ P. Poccetti, Nuovi document italici a complemento del Manuale di E. Vetter (Orientamenti linguistici 8), Pisa 1979.

Poco grano, molti frutti $=$ A. Campanelli (Ed.), Poco grano, molti frutti. 50 anni di archeologia ad Alba Fucens, Sulmona (AQ) 2006.

Poggio Mirteto = P. Armellin (Ed.), La storia del territorio di Poggio Mirteto. Un racconto con il contributo di varie discipline. Atti della giornata di studi, Poggio Mirteto, 29 novembre 2014, Monte Compatri (RM) 2018.

Polito, Fulgentibus armis = E. PolIto, Fulgentibus armis. Introduzione allo studio dei fregi d'armi antichi (Xenia antiqua. Monografie 4), Roma 1998.

Pompei = F. Senatore (Ed.), Pompei, Capri e la Penisola Sorrentina. Atti del quinto ciclo di conferenze di geologia, storia e archeologia, Pompei, Capri, Scafati, Castellammare di Stabia, ottobre 2002 - aprile 2003, Capri (NA) 2004.

Ponte Romano = T. Leggio - R. Lorenzetti - E. M. Menotti (Ed.), Il ponte romano sul Velino (Quaderni di storia urbana e territoriale 2), Rieti 1988.

Popoli dell'Italia antica $=$ Popoli dell'Italia antica. Gentes fortissimae Italiae. Samnium, Latium et Campania. Le antiche città scomparse. Atti del $2^{\circ}$ Convegno, San Vittore del Lazio, 28 ottobre 2007 (Quaderni di studi 2. Historia 17), Formia (LT) 2007

Pouthier, Ops = P. Pouthier, Ops et la conception divine de l'abondance dans la religion romaine jusqu'à la mort d'Auguste (Bibl. des Écoles Fr. d'Athènes et de Rome 242), Rome 1981.

Praxis municipale $=\mathrm{L}$. LAMOINe - C. Berrendonner $-\mathrm{M}$. Cébeillac-Gervasoni (Ed.), La Praxis municipale dans l'Occident romain, Paris 2010.

Prénoms = P. PocCETTI (Ed.), Les prénoms de l'Italie antique. Journée d'études, Lyon, 26 janvier 2004 (Ricerche sulle lingue di frammentaria attestazione 5), Pisa 2008.

Priori, La Frentania $=$ D. Priori, La Frentania I, Lanciano (CH) 1942.

Proprietà imperiali $=$ D. PuPILLO $($ Ed.), Le proprietà imperiali nell'Italia romana. Economia, produzione, amministrazione. Atti del Convegno, Ferrara - Voghiera, 3-4 gingno 2005 (Quaderni degli Annali dell'Università di Ferrara. Sezione Storia 6), Firenze 2007.

Prosopographie = W. Eck - M. HeIL (Ed.), Prosopographie des römischen Kaiserreichs: Ertrag und Perspektiven. Kolloquium aus Anlass der Vollendung der Prosopographia Imperii Romani, Berlin, 27.-28. 10. 2016, Berlin 2017.

Prosopographie und Sozialgeschichte $=$ W. EcK (Ed.), Prosopographie und Sozialgeschichte. Studien zur Methodik und Erkenntnismöglichkeit der kaiserzeitlichen Prosopographie. Kolloquium Köln 24.-26. November 1991, Köln 1993.

Prosperi Valenti, Valetudo = G. Prosperi Valenti, Valetudo. Origine ed aspetti del culto nel mondo romano (Stud. Ist. It. Stor. Ant. 67), Roma 1998.

Public Space $=$ Chr. P. Dickenson - O. M. van Nijf (Ed.), Public Space in the Post-Classical City. Proceedings of a One Day Colloquium held at Fransum 23 ${ }^{\text {rd }}$ July 2007 (Caeculus 7), Leuven 2013.

Purdie, Latin Verse = A. B. Purdie, Latin Verse Inscriptions, London 1935.
Quadrifluus amnis = Quadrifluus amnis. Studi di letteratura, storia, filosofia e arte offerti dalla Facoltà di Lettere e Filosofia dell'Università di Chieti a mons. Costantino Vona, Chieti 1987.

Quilici, Collatia $=$ L. Quilici, Collatia (Forma Italiae. Regio I. Vol. X), Roma 1974.

Quotidien municipal $=$ C. Berrendonner - M. CéBeIllaCGeRVASONI - L. LAMOINe (Ed.), Le Quotidien municipal dans l'Occident romain. Actes du colloque international tenu à Clermont-Ferrand et à Chamalières du 19 au 21 octobre 2007, Paris 2008.

RADKe, Die Götter Altitaliens ${ }^{2}=$ G. RadKe, Die Götter Altitaliens $^{2}$ (Fontes et Commentationes 3), Münster 1979.

Raepsaet-Charlier, Clarissima femina $=$ M.-Th. RaepsaetCHARLIER, Clarissima femina. Études d'histoire sociale des femmes de l'élite à Rome. Scripta varia. Travaux rassemblés et édités par A. Álvarez Melero (Inst. Hist. Belge de Rome. Études 3), Bruxelles - Rome 2016.

Raepsaet-Charlier, Prosopographie des femmes = M.-Th. Raepsaet-Charlier, Prosopographie des femmes de l'ordre sénatorial (I ${ }^{e r}-I I^{e}$ siècles) (Académie Royale de Belgique, Classe des Lettres 4), Lovanii 1987.

Ragusa, Brevi cenni = M. Ragusa, Brevi cenni sulla Marsica, Bologna 1924.

Raimo, Storia $=$ L. C. Raimo, Storia del territorio sampelinese (Quaderni Sampelinesi), Avezzano (AQ) 2006.

RAMELli, Cultura $=$ I. RAMELli, Cultura e religione etrusca nel mondo romano. La cultura etrusca dalla fine dell'indipendenza, Alessandria 2003.

RANIERI, Guardiagrele = F. P. RANIERI, Guardiagrele. Memorie e monumenti paesani. Prima edizione raccolta ed ordinata da RANUCCIO RANIERI, illustrata con 100 fotoincisioni fuori testo, con prefazione di Corrado Ricci, Lanciano (CH) 1927.

Rapisarda, Monoliti $=$ A. Rapisarda, I monoliti-torchio dell'Abruzzo aquilano e il culto di Ercole, Sesto Fiorentino (FI) 2017.

RAPISARDA, Vestini $=$ A. RaPISARDA, I Vestini e il mistero del pagus di Separa: una ricerca sulla più grande cinta fortificata del territorio dei Vestini Cismontani, L'Aquila 2011.

Raspini, Mompeo = F. Raspini, Cenni storici e appunti su Mompeo, Roma 1977.

Rawson, Children $=$ B. Rawson, Children and Childhood in Roman Italy, Oxford 2003.

$R E=$ Paulys Realencyclopädie der classischen Altertumswissenschaft I - Suppl. XV, Stuttgart 1893-1978.

Reali, Amicitia = M. Reali, Il contributo dell'epigrafia latina allo studio dell'amicitia: il caso della Cisalpina (Pubblicazioni della Facoltà di Lettere e Filosofia dell'Università degli Studi di Milano 176. Sezione di Storia Antica 2), Firenze 1998.

Reate e l'Ager Reatinus = F. COARELli - A. De SANTIS (Ed.), Reate e l'Ager Reatinus. Vespasiano e la Sabina: dalle origini all'Impero. Catalogo della mostra (Rieti, Museo Civico - Sezione Archeologica, 8 maggio - 22 novembre 2009), Roma 2009

Recherches sur les structures sociales = Recherches sur les structures sociales dans l'antiquité classique. Caen, 25-26 avril 1969 (Colloque Nationaux du Centre National de la Recherche Scientifique), Paris 1970.

Redaelli, Veterani $=$ D. Redaelli, I veterani delle milizie urbane in Italia e nelle province di lingua latina. Indagine storico-epigrafica (liber machina dactylographica redactus thesis doctoris dignitatis nominisque a. 2013/14 Tergeste impetratae apud Università degli Studi di Trieste).

REDDÉ, Mare nostrum = M. REDDÉ, Mare nostrum. Les infrastructures, le dispositif et l'histoire de la marine militaire sous l'empire romain (Bibl. des Écoles Fr. d'Athènes et de Rome 260), Roma 1986. 
Reggiani, Museo Civico $^{1}=$ A. M. Reggiani, Rieti. Museo Civico. Rinvenimenti della città e del territorio ${ }^{1}$ (Cataloghi 2), Roma 1981.

Reggiani Massarini, Museo Civico ${ }^{2}=$ A. M. Reggiani MasSarini, Museo Civico di Rieti ${ }^{2}$ (Cataloghi 7), Roma 1990.

Reggiani Massarini, Reate $=$ A. M. Reggiani Massarini, Rieti in età romana, in M. C. Spadoni Cerroni - A. M. Reggiani Massarini, Reate [I.] (Bibl. Stud. Antichi 68), Pisa 1992, 125-190.

Reggiani Massarini, Santuario degli Equicoli = A. M. Reggiani Massarini, Santuario degli Equicoli a Corvaro. Oggetti votivi del Museo Nazionale Romano (Lavori e Studi 11), Roma 1988.

Reimpiego in architettura $=\mathrm{J}$. -FR. BERNARD - PH. BERNARDI D. Esposito (Ed.), Il reimpiego in architettura. Recupero, trasformazione, uso. Atti del convegno, Roma, 8-10 novembre 2007 (Coll. de l'École Fr. de Rome 418), Rome 2008.

Renovación = A. F. Caballos Rufino (Ed.), Del municipio a la corte. La renovación de las élites romanas (Historia y Geografía 208), Sevilla 2012.

RIB = The Roman Inscriptions of Britain

C. Ricci, Cenotafi = C. RiccI, Qui non riposa. Cenotafi antichi e moderni fra memoria e rappresentazione (Libitina 4), Roma 2006.

C. RiccI, Security = C. RiccI, Security in Roman Times. Rome, Italy and the Emperors, London - New York 2018.

C. RIccI, Soldati $=$ C. RIccI, Soldati delle milizie urbane fuori di Roma. La documentazione epigrafica (Opuscula epigraphica 5), Roma 1994.

C. RICCI, Soldati e veterani $=$ C. RICcI, Soldati e veterani nella vita cittadina dell'Italia imperiale (Urbana species 1), Roma 2010.

E. Ricci, Bosco sacro ${ }^{2}=$ E. Ricci, Il bosco sacro del Sirente fra Sicinnide e leggenda ${ }^{2}$, Sulmona (AQ) 1989.

E. RICCI, Elementi $=$ E. RICCI, Elementi di civiltà dei Peligni Superequani, Sulmona (AQ) 1978.

E. RICCI, Epigrafi $=$ E. RICCI, Epigrafi superequane che camminano, Sulmona (AQ) 1987.

E. RICCI, Esegesi = E. RiccI, Esegesi epigrafica di Superaequum, Sulmona (AQ) 1989.

E. RIccI, Nuova esegesi = E. RICcI, La tomba di Scribonia a Superaequum e nuova esegesi epigrafica, Sulmona (AQ) 1991.

E. RiccI, Peligni Superequani = E. RiccI, I Peligni Superequani, la Sicinnide e le origini di Secinaro, Sulmona (AQ) 1969.

E. Ricci, Secinaro = E. Ricci, Secinaro in Abruzzo dal Paleolitico a Superaequum. Archeologia e pseudoarcheologia nella Valle Subequana, Sulmona (AQ) 2000.

E. RICCI, Superaequum = E. RICCI, Superaequum e gli antich Cedici, Pratola Peligna (AQ) 1981.

E. RiccI, Ubicazione = E. RiccI, Ubicazione di Superaequum e spigolature peligne, Sulmona (AQ) 1984

Riccomanni, $m s .=$ L. E. Riccomanni, Iscrizioni copiate su' loro medesimi Autografi da L. E. Riccom(an)ni ne varj viaggi fatti del med(esim)o in diversi luoghi d'Italia (libellus p. 101-106 inter a. 1759/67 exaratus, insertus in volumine, cui titulus Manuale scientifico, dove sono notati moltissimi fragmenti sopra varie scienze esposti da vari autori coll'aggiunta di scienze politiche, economiche e militari, nunc servato Treia in bibliotheca Accademia Georgica).

Ricerche archeologiche = D. GABLER - F. REDÖ (Ed.), Ricerche archeologiche a San Potito di Ovindoli e le aree limitrofe nell'antichità e nell'alto medioevo. Atti del convegno sui problemi archeologici di una villa romana nella Marsica, Budapest, 24 novembre 2000, L'Aquila 2008.
Richier, Centuriones = O. Richier, Centuriones ad Rhenum. Les centurions légionnaires des armées romaines $d u$ Rhin (Gallia Romana 6), Paris 2004.

RicL, Inscriptions = M. RICL, The Inscriptions of Alexandreia Troas (Die Inschriften griechischer Städte aus Kleinasien 53), Bonn 1997.

Ricordo di Filippo Càssola = M. ChiabÀ - L. Cristante (Ed.), Il sussurro di una brezza leggera. Ricordo di Filippo Càssola (Polymnia 6), Trieste 2017.

Ricotтi, Castelvecchio Subequo = E. RicotтI, Castelvecchio Subequo. Brevi notizie storiche, Ancona 1961.

Ritterling, Fasti $=$ E. RitTerling, Fasti des römischen Deutschland unter dem Prinzipat, Wien 1932.

Ritschl, Monumenta $=$ F. RitsChL, Priscae Latinitatis monumenta epigraphica, Berlin 1862.

RIVERA, ms. = G. RIVERA, Breviarium manu scriptum a. 1893 servatum Romae apud Archivio Centrale dello Stato (EUR). MPI. Dir. Gen. AABBAA. Antichità e Scavi. II versamento, $1^{\circ}$ serie, $b .14$, fasc. 238.

Rivoluzione Augusto = R. PARIs - S. BRUNI - M. Roghi (Ed.), Rivoluzione Augusto: l'imperatore che riscrisse il tempo e la città, 17 dicembre 2014 - 2 giugno 2015, Milano 2015.

Rix, Cognomen = H. Rix, Das etruskische Cognomen, Wiesbaden 1963.

RIx, Sabellische Texte = H. RIx, Sabellische Texte. Die Texte des Oskischen, Umbrischen und Südpikenischen (Handbuch der italischen Dialekte 5), Heidelberg 2002.

RizzeLLo, Culti orientali $=$ M. Rizzello, I culti orientali nella Media Valle del Liri, Sora (FR) 1984.

ROBERT, Gladiateurs = L. ROBERT, Les gladiateurs dans l'Orient grec (Bibl. de l'École Haut. Ét. Sc. Hist. 278), Paris 1940.

Rocchia, Guglionesi $=$ A. M. RocchiA, Cronistoria $d i$ Guglionesi e delle tre gloriose traslazioni di s. Adamo abate suo protettore, Napoli 1890 (lucis ope iterum impressum Vasto (CH) 1991, curante G. Morlachetti).

Rockwell, Private Baustiftungen = J. C. Rockwell, Private Baustiftungen für die Stadtgemeinde auf Inschriften der Kaiserzeit im Westen des römischen Reiches, Diss. Jena 1909.

Roddaz, Agrippa = J.-M. Roddaz, Marcus Agrippa (Bibl. des Écoles Fr. d'Athènes et de Rome 253), Paris - Roma 1984.

Römische Gräberstraßen = H. vON HESBERG - P. ZANKER (Ed.), Römische Gräberstraßen. Selbstdarstellung - Status - Standard. Kolloquium in München vom 28. bis 30. Oktober 1985 (Bayer. Akad. d. Wiss., Phil.-hist. Kl., Abh., N. F. 96), München 1987.

Rohlfs, Sermo Vulgaris ${ }^{2}$ = G. Rohlfs, Sermo Vulgaris Latinus. Vulgärlateinisches Lesebuch ${ }^{2}$ (Sammlung kurzer Lehrbücher der romanischen Sprachen und Literaturen 13), Tübingen 1956.

Roldán Hervás, Hispania y el ejército = J. M. Roldán HeRvás, Hispania y el ejército romano. Contribución a la historia social de la España antigua (Acta Salmaticensia, Filosofia y Letras 76), Salamanca 1974.

Roma medio repubblicana $=$ Roma medio repubblicana . Aspetti culturali di Roma e del Lazio nei secoli IV e III a. C., Roma 1973.

Roma repubblicana $=$ A. M. REgGIANI (Ed.), Roma repubblicana dal 270 a. C. all'età augustea (Archeologia e storia a Roma [3]), Roma 1987.

Roman Army in the East = D. L. Kennedy (Ed.), The Roman Army in the East (JRA. Supplementary Series 18), Ann Arbor (MI) 1996.

Roman Baths = J. De Laine - D. E. Johnston (Ed.), Roman Baths and Bathing. Proceedings of the First International Conference on Roman Baths held at Bath, England, 30 March - 4 April 1992 (JRA. Supplementary Series 37), Portsmouth 1999 . 
Roman Empire = K. KŁodZIŃskI et AL. (Ed.), The Roman Empire in the Light of Epigraphical and Normative Sources (Studies in Greek and Roman History 4), Toruń 2013.

Roman Imperial Cities = A. ANDRADE et AL. (Ed.), Roman Imperial Cities in the East and in Central-Southern Italy (Ancient Cities 1), Roma 2019.

Roman Middle Republic = CHR. BruUn (Ed.), The Roman Middle Republic. Politics, Religion, and Historiography c. 400-133 B.C. Papers from a conference at the Institutum Romanum Finlandiae, September 11-12, 1998 (Acta Inst. Rom. Finl. 23), Roma 2000.

Roman Miscellany $=$ H. M. SCHellenberg - V. E. HirschMANN - A. Krieckhaus (Ed.), A Roman Miscellany. Essays in Honour of Anthony R. Birley on his Seventieth Birthday (Akanthina 3), Gdańsk 2008.

Roman Republican Colonization = T. D. STeK - J. Pelgrom (Ed.), Roman Republican Colonization. New Perspectives from Archaeology and Ancient History (Papers of the Royal Netherlands Institute in Rome 62), Rome 2014.

Romanelli, Antica topografia = D. Romanelli, Antica topografia istorica del Regno di Napoli I-III, Napoli 1815/19.

Romanin, Fiamignano $=$ H. Romanin, Fiamignano e la sua storia, Rieti 1983.

Romanisation du Samnium = La romanisation du Samnium aux $I I I^{e}$ et $I^{e r}$ siècles av. I.-C. Actes du colloque organisé par le Centre Jean Bérard en collaboration avec la Soprintendenza archeologica e per i BAAAS del Molise et la Soprintendenza archeologica per le province di Salerno, Avellino e Benevento. Naples - Centre Bérard, 4-5 novembre 1988 (Bibl. de l'Inst. Franç. Naples ser. 2, 9), Naples 1991.

Romanitas - Christianitas = G. WIRTH et AL. (Ed.), Romanitas - Christianitas. Untersuchungen zur Geschichte und Literatur der römischen Kaiserzeit. Johannes Straub zum 70. Geburtstag am 18. Okt. 1982 gewidmet, Berlin - New York 1982.

Romano, Solopaca ${ }^{1}$ = A. Romano, La nostra terra. Storia di Solopaca ${ }^{1}$, Napoli 1977.

Romano, Solopaca ${ }^{2}$ = A. Romano, La nostra terra. Storia di Solopaca ${ }^{2}$. In appendice: C. Formichella, Piccolo dizionario biografico dei solopachesi illustri, Piedimonte Matese (CE) 1998.

Romeo, Ingenuus Leo = I. Romeo, Ingenuus Leo. L'immagine di Agrippa (Xenia Antiqua. Monografie 6), Roma 1998.

Rosa, ms. I = [G.?] Rosa, Selva di molte memorie di Norcia scritte senza ordine di tempi. Parte I (liber inter a. 1641 et a. 1675 manu scriptus in aedibus privatis oppidi Acquasparta adservatus, f. $50 \mathrm{v}-122 \mathrm{v}$ ).

Rosa, ms. II = [G.?] Rosa, Selva di molte memorie di Norcia scritte senza ordine di tempi. Parte II (liber manus scriptus inter a. 1641 et a. 1675 adservatus Perugia in Biblioteca comunale Augusta sub n. 3312 int. 2).

$\mathrm{CH}$. Rose, Dynastic Commemoration $=\mathrm{CH}$. B. Rose, Dynastic Commemoration and Imperial Portraiture in the JulioClaudian Period, Cambridge 1997.

D. Rose, Studi = D. Rose, Studi sull'opera poligonale tra alta Valle del Salto e Fucino. L'acquedotto e la cloaca maxima di Alba Fucens (Misura e Spazio 1), Roma 2018.

Rossi, $m s .=$ G. C. Rossi, Lapide Marsicane raccolte ed osser vate diligentemente dall'Ill.mo e R.mo Monsignor $D$. Gio<vanNi> Camillo Rossi (liber temporis spatio inter a. 1806 et a. 1818 comprehenso manu exaratus in Bibliotheca Apostolica Vaticana adservatus sign. Ferrajoli 513; vide BuONocore, Misc. Bibl. Ap. Vat. 1, 1987, 33-53).

Rostovtzeff e l'Italia = A. MARCONE (Ed.), Rostovtzeff e l'Italia. Gubbio, Casa di Sant'Ubaldo, 25-27 maggio 1995 (Incontri perugini di storia della storiografia antica e del mondo antico 9. Studi di storia e di storiografia 5), Perugia 1999.
Rostovzev, Storia economica ${ }^{5}=\mathrm{M}$. Rostovzev, Storia economica e sociale dell'Impero romano ${ }^{5}$ (Il pensiero storico 3), Bologna - Firenze 1973.

Royden, Magistrates = H. L. Royden, The Magistrates of the Roman Professional Collegia in Italy from the First to the Third Century A. D. (Bibl. Stud. Antichi 61), Pisa 1988.

Rubini, Storia di Penne = A. Rubini, Storia di Penne, Penne (AQ) 1989.

Rudolph, Stadt und Staat $=$ H. Rudolph, Stadt und Staat im römischen Italien. Untersuchungen über die Entwicklung des Munizipalwesens in der republikanischen Zeit, Leipzig 1935.

RÜPKE, Kalender = J. RüPKE, Kalender und Öffentlichkeit. Die Geschichte der Repräsentation und religiösen Qualifikation von Zeit in Rom (RGVV 40), Berlin - New York 1994.

RUOFF-VÄÄNÄNEN, Italian fora $=$ E. RUOFF-VÄÄNÄNEN, Studies on the Italian fora (Historia Einzelschriften 32), Stuttgart 1978.

Rupes loquentes = L. GASPERINI (Ed.), Rupes loquentes. Atti del Convegno internazionale di studi sulle "Iscrizioni rupestri di età romana in Italia”, Roma - Bomarzo, 13-15. X. 1989 (Stud. Ist. It. Stor. Ant. 53), Roma 1992.

Russi, Teanum Apulum = A. Russi, Teanum Apulum. Le iscrizioni e la storia del municipio (Stud. Ist. It. Stor. Ant. 25), Roma 1976.

Sabbatini Tumolesi, Gladiatorum paria $=$ P. Sabbatini Tumolesi, Gladiatorum paria. Annunci di spettacoli gladiatorii a Pompei (Tituli 1), Roma 1980.

Sabbatucci, Religione $=$ D. SABbatucci, La religione $d i$ Roma antica: dal calendario festivo all'ordine cosmico, Milano 1988 .

Sabini = G. Alvino (Ed.), I Sabini. La vita, la morte, gli dei. Catalogo della mostra. Rieti, Sala dei Cordari, 30 ottobre - 15 dicembre 1997, Roma 1997.

SABLAyrolles, Libertinus miles $=$ R. SABLAYRolles, Libertinus miles. Les cohortes de vigiles (Coll. de l'École Fr. de Rome 224), Paris - Roma 1996.

SACCHETTI SASSETTI, Ultime vicende $=$ A. SACCHETTI SASSETTI, Le ultime vicende di un antico monumento reatino, Rieti 1952.

Saddington, Development $=$ D. B. SAddington, The Development of the Roman Auxiliary Forces from Caesar to Vespasian (49 B. C. - A. D. 78), Harare 1982.

Saeculum Aureum = I. Baglioni (Ed.), Saeculum Aureum. Tradizione e innovazione nella religione romana di epoca augustea I-II (Religio. Collana di Studi del Museo delle Religioni "Raffaele Pettazzoni” [4]), Roma 2016.

Saepinum = Saepinum. Museo documentario dell'Altilia, Campobasso 1982.

Safinim = D. CAIAZZA (Ed.), Safinim. Studi in onore di Adriano La Regina per il premio "I Sanniti" (Libri Campano-Sannitici 3), Piedimonte Matese (CE) 2004.

SALAČ, Isis = A. SALAČ, Isis, Sarapis a božstva sdružená dle svědectví řeckcých a latinských nápisù, Praha 1915.

Saletta, S. Anatolia = V. Saletta, S. Anatolia, Roma [1968]. Saline e sale $=\mathrm{S}$. Russo $-\mathrm{R}$. Goffredo (Ed.), Saline e sale nell'antichità. Atti del III Convegno sul Basso Tavoliere (Documenti e studi 69), Bari 2018.

Salomies, Nomenclature $=$ O. Salomies, Adoptive and Polyonymous Nomenclature in the Roman Empire (Soc. Scient. Fennica, Comment. Human. Litt. 97), Helsinki 1992.

Salomies, Vornamen $=$ O. SAlomies, Die römischen Vornamen. Studien zur römischen Namengebung (Soc. Scient. Fennica, Comment. Human. Litt. 82), Helsinki 1987.

Samnitice loqui I-II = D. CaIazza (Ed.), Samnitice loqui. Studi in onore di Aldo L Prosdocimi per il premio "I Sanniti” I- 
II (Libri Campano-Sannitici 5), Piedimonte Matese (CE) 2006.

Samnium = S. CAPINI - A. Di Niro (Ed.), Samnium. Archeologia del Molise, Roma 1991.

San Potito Sannitico = N. Lombardi (Ed.), San Potito Sannitico da villaggio rurale a città del parco, Piedimonte Matese (CE) [2004].

San Vincenzo al Volturno = J. Mitchell - I. L. Hansen C. M. CoutTs (Ed.), San Vincenzo al Volturno 3: The Finds from the 1980-1986 Excavations I-II (Spoleto. Centro Italiano di studi sull'Alto Medioevo. Studi e ricerche di archeologia e storia dell'arte 3), Spoleto (PG) 2001.

Sanctuaires = O. DE CAZANOVE - J. SCHEID (Ed.), Sanctuaires et sources dans l'antiquité. Les sources documentaires et leurs limites dans la description des lieux de culte. Actes de la table ronde organisée par le Collège de France, l'UMR 8585 Centre Gustave-Glotz, l'École Française de Rome et le Centre Jean Bérard. Naples - Centre Jean Bérard, 30 novembre 2001, Napoli 2003.

SANDERS, Lapides memores $=\mathrm{G}$. SANDERS, Lapides memores Païens et chrétiens face à la mort: le témoignage de l'épigraphie funéraire latine (Epigrafia e Antichità 11), Bologna - Faenza 1991.

Sangue e arena $=$ A. LA Regrina (Ed.), Sangue e arena, Milano 2001.

Sannio = Sannio. Pentri e Frentani dal VI al I sec. a. C. Isernia, Museo Nazionale, ottobre - dicembre 1980, Roma 1980.

SANSI, Edifici $=$ A. SANSI, Degli edifici e dei frammenti storici delle antiche età di Spoleto, Foligno (PG) 1869 (lucis ope iterum impressum Perugia 1972).

SANTA Maria Scrinari, Alba Fucens $=$ V. SANTA Maria ScriNARI, Alba Fucens di Massa d'Albe (Quad. Aquilano 3), Terni 1978.

Santarelli, Voci e ricordi $=$ E. Santarelli, Voci e ricordi della mia terra, Amatrice (RI) 1927.

Santilli, Aquae Cutiliae = E. Santilli, Aquae Cutiliae. Tempus tantum nostrum est, Rieti 2016.

SANTINELli, Santuari = A. SANTINELli, Santuari medio-repubblicani di Ercole dell'Italia centro-appenninica (liber machina dactylographica redactus thesis doctoris dignitatis nominisque a. 2012/13 Macerata impetratae apud Università degli Studi. Facoltà di Lettere e Filosofia).

Santuario = Il Santuario di Vescovio nella Storia, nella Fede, nell'Arte, Monterondo Scalo (RM) [2000].

SARtori, Dall'Italía $=$ F. SARtori, Dall'Italía all'Italia I-II (Saggi e materiali universitari 27. Serie di antichità e tradizione classica 19), Padova 1993.

Saxa scripta I = A. Rodríguez Colmenero - L. Gasperini (Ed.), Saxa scripta. Inscripciones en Roca. Actas del Simposio Internacional Ibero-Itálico sobre epigrafía rupestre. Santiago de Compostela y Norte de Portugal, 29 de junio a 4 de julio de 1992 (Anejos de Larouco 2), Sada 1995.

Saxa Scripta III = Saxa Scripta. Actas do III Simpósio IberoItálico de epigrafia rupestre. Viseu, 3-5 de abril de 1997, Viseu 2001.

Sblendorio Cugusi, Composti nominali $=$ M. T. SblendoRIo Cugusi, L'uso stilistico dei composti nominali nei Carmina Latina Epigraphica (Quaderni di "Invigilata lucernis” 25), Bari 2005.

SCARPELLINI, Stele romane $=$ D. SCARPellini, Stele romane con imagines clipeatae in Italia (Stud. Arch. 46), Roma 1987.

SCenna, Archeologia teatina $=$ D. SCEnNA, Archeologia teatina. Esperienze, delusioni, soddifazioni di un R. ispettore onorario dei monumenti e scavi (Annuario del Reale Liceo Ginnasio "G. B. Vico" per gli anni 1934/35 e 1935/36), Chieti 1937.
SCHÄFER, Imperii insignia $=$ TH. SCHÄFER, Imperii insignia: sella curulis und fasces. Zur Repräsentation römischer Magistrate (RM, Ergänzungsheft 29), Mainz 1989.

SChallmayer et Al., Beneficiarii $=$ E. SChallmayer - K. Eibi - J. OtT - G. Preuss - E. WitTkopf, Der römische Weibebezirk von Osterburken 1. Corpus der griechischen und lateinischen Beneficiarier-Inschriften des römischen Reiches (Forschungen und Berichte zur Vor- und Frühgeschichte in Baden-Württemberg 40), Stuttgart 1990.

Scheid, Les Frères Arvales = J. Scheid, Les Frères Arvales. Recrutement et origine sociale sous les empereurs julioclaudiens (Bibl. de l'École Haut. Ét. Sc. Rel. 77), Paris 1975.

SCHEID, Romulus et ses frères = J. SCHEID, Romulus et ses frères. Le collège des Frères Arvales, modèle du culte public dans la Rome des empereurs (Bibl. des Écoles Fr. d'Athènes et de Rome 275), Paris - Roma 1990.

Schiavi d'Abruzzo = S. Lapenna (Ed.), Schiavi d'Abruzzo. Le aree sacre, Sulmona (AQ) 2006.

SCHNeIDer, Dialecti $=$ E. SchneIder, Dialecti Latinae Priscae et Faliscae exempla selecta I Dialecti Latinae Priscae et Faliscae inscriptiones, Lipsiae 1886.

SCHNORR VON CAROLSFELD, Geschichte der juristischen Person $=\mathrm{L}$. SCHNORR VON CAROLSFELD, Geschichte der juristischen Person I. Universitas, Corpus, Collegium im klassischen römischen Recht, München 1933 (ed. altera Aalen 1966).

SCHÖRNER, Römische Rankenfriese = G. SCHÖRNER, Römische Rankenfriese. Untersuchungen zur Baudekoration der späten Republik und der frühen und mittleren Kaiserzeit im Westen des Imperium Romanum (Beiträge zur Erschliessung hellenistischer und kaiserzeitlicher Skulptur und Architektur 15), Mainz 1995.

Schulze, Eigennamen $=$ W. Schulze, Zur Geschichte lateinischer Eigennamen (Abh. d. Akad. d. Wiss. Göttingen, Phil.-hist. Kl., N. F. 5, 5), Berlin 1904 (lucis ope iterum impressum Berlin 1933, lucis ope tertium impressum mit einer Berichtigungsliste zur Neuausgabe von O. SALOMIES, Zürich - Hildesheim 1991).

Schumacher, Priesterkollegien = L. Schumacher, Prosopographische Untersuchungen zur Besetzung der vier hohen römischen Priesterkollegien im Zeitalter der Antoninen und der Severer (96-235 n. Chr.), Mainz 1973.

Sclocchi, Storia dei Marsi = R. Sclocchi, Storia dei Marsi, dalle età più antiche al 1911 I. Storia dei Marsi antichi, Aquila 1911.

Scritti Carlo Pietrangeli $=$ V. Casale - F. Coarelli - B. Toscano (Ed.), Scritti di archeologia e storia dell'arte in onore di Carlo Pietrangeli (Studi dell'Accademia Spoletina 9), Roma 1996.

Scritti Fulvio Grosso = L. GASPERINI (Ed.), Scritti sul mondo antico in memoria di Fulvio Grosso (Università di Macerata. Pubblicazioni della Facoltà di Lettere e Filosofia 9), Roma 1981.

Scritti Gaetano Messineo = E. Mangani - A. Pellegrino

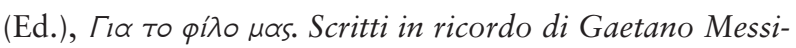
neo, Roma 2016.

Scritti Gino Bandelli = M. СнiabÀ (Ed.), Hoc quoque laboris praemium. Scritti in onore di Gino Bandelli (Polymnia 3), Trieste 2014.

Scritti Gioia Conta = U. LAFFi - F. Prontera - B. Virgilio (Ed.), Artissimum memoriae vinculum. Scritti di geografia antica e di antichità in ricordo di Gioia Conta, Firenze 2004.

Scritti Giovanni Uggeri = C. MARANGIO - G. LAudizi (Ed.),

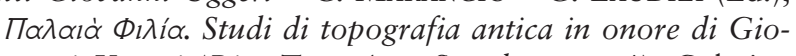
vanni Uggeri (Riv. Top. Ant. Supplemento 4), Galatina (LE) 2009. 
Scritti Lorenzo Braccesi = F. Raviola - M. BAssi - A. Debiasi - E. Pastorio (Ed.), L'indagine e la rima. Scritti per Lorenzo Braccesi (Hesperia 30), Roma 2013.

Scritti Marcello Zambelli = L. GASPERINI (Ed.), Scritti storicoepigrafici in memoria di Marcello Zambelli (Università di Macerata. Pubblicazioni della Facoltà di Lettere e Filosofia 5), Roma 1978.

Scritti Maria Pia Rossignani = S. Lusuardi Siena et AL. (Ed.), Archeologia classica e post-classica tra Italia e Mediterraneo. Scritti in ricordo di Maria Pia Rossignani (Contributi di archeologia 8), Milano 2016.

Scritti Mario Pani $=$ S. Cagnazzi et AL. (Ed.), Scritti di storia per Mario Pani (Documenti e studi 48), Bari 2011.

Scritti Salvatore Alessandrì = M. LOMBARDO - C. MARANGIO (Ed.), Antiquitas. Scritti di storia antica in onore di Salvatore Alessandrì, Galatina (LE) 2011.

Scritti Sándor Soproni = A. Szaвó - E. Tо́тн (Ed.), Bölcske: römische Inschriften und Funde. In memoriam Sándor Soproni (1926-1995) (Libelli archaeologici 2), Budapest 2003.

Scullard, Scipio Africanus = H. H. Scullard, Scipio Africanus: Soldier and Politician, London 1970.

Sculture = A. Giuliano (Ed.), Museo Nazionale Romano. Le sculture I 1, Roma 1979. I 2, Roma 1981. I 3, Roma 1982. I 4, Roma 1983. I 5, Roma 1983. I 6, Roma 1986. I 7, 1 2, Roma 1984. I 8, 1-2, Roma 1985. I 9, 1-2, Roma $1987 /$ 88. I 10, 1-2, Roma 1988/95. I 11, Roma 1991.

Scurcola Marsicana I = G. Grossi - R. Colapietra - F. D'Amore, Scurcola Marsicana. Historia, Scurcola Marsicana (AQ) 2005.

Scurcola Marsicana II = G. GrossI, Scurcola Marsicana. Monumenta, Scurcola Marsicana (AQ) 2006.

Seaborne Commerce = J. K. D’Arms - E. C. KopfF (Ed.), The Seaborne Commerce of Ancient Rome: Studies in Archaeology and History (Mem. Amer. Acad. Rome 36), Rome 1980.

SEAR, Roman Theatres = F. SEAR, Roman Theatres. An Architectural Study (Oxford Monographs on Classical Archaeo$\log y)$, Oxford 2006

SEgENNI, Amiternum $=\mathrm{S}$. SEgENNI, Amiternum e il suo territorio in età romana (Bibl. Stud. Antichi 49), Pisa 1985.

Segenni, Decreta Pisana = S. Segenni, I Decreta Pisana. Autonomia cittadina e ideologia imperiale nella colonia Opsequens Iulia Pisana (Documenti e studi 47), Bari 2011.

Segenni, Liberti $=$ S. Segenni, I liberti di Amiternum. Ricerche di onomastica (Bibl. Stud. Antichi 59), Pisa 1990.

Segni dei mestieri = M. D’Amadio (Ed.), I Segni dei mestieri. Banchi, grida, insegne. Catalogo della mostra. Roma Museo Nazionale delle arti e tradizioni popolari (Roma, 10 giugno - 31 dicembre 1992), Roma 1992

Segni sulla pietra = E. MatтIOcco (Ed.), Segni sulla pietra . Iscrizioni e araldica dalla terra di Castel di Sangro (Novitas 3), Lanciano (CH) 2003.

Senados municipales $=\mathrm{E}$. Melchor GiL $-\mathrm{A} . \mathrm{D}$. Pérez Zurita - J. F. Rodríguez Neila (Ed.), Senados municipales y decuriones en el Occidente romano (Historia y Geografía 249), Sevilla 2013

Senatores populi Romani $=$ W. ECK - M. HeIL (Ed.), Senatores populi Romani. Realität und mediale Präsentation einer Führungsschicht. Kolloquium der Prosopographia Imperi Romani vom 11.-13. Juni 2004 (HABES 40), Stuttgart 2005.

Sentino = D. Poli (Ed.), La battaglia del Sentino. Scontro fra nazioni e incontro in una nazione. Atti del Convegno di studi, Camerino - Sassoferrato, 10-13 giugno 1998 (Ouaderni linguistici e filologici 14), Roma 2002.

Sepino = Sepino. Archeologia e continuità, Campobasso 1979.

Sestinum = Sestinum. Comunità antiche dell'Appennino tra Etruria e Adriatico. Atti del Convegno tenuto a Sestino
(Arezzo), 18-19 settembre 1983 (Collana Volusenus. Testi e studi promossi dalla Biblioteca Comunale di Sestino 2), Rimini 1989.

Seston, Scripta varia $=\mathrm{W}$. Seston, Scripta varia. Mélanges d'histoire romaine, de droit, d'épigraphie et d'histoire du christianisme (Coll. de l'École Fr. de Rome 43), Paris Roma 1980.

Settefrati, S. Pietro Avellana = P. Settefrati, Volana oggi S. Pietro Avellana dalle origini ai nostri giorni, Teramo 2000.

Settipani, Continuité gentilice $=$ Chr. Settipani, Continuité gentilice et continuité familiale dans les familles sénatoriales romaines à l'époque impériale. Mythe et réalité (Prosopographica et Genealogica 2), Oxford 2000.

Seyfried, Ethnika = E. SEYFried, Die Ethnika des alten Italiens, Zürich 1951

Sherk, Municipal Decrees = R. K. Sherk, The Municipal Decrees of the Roman West (Arethusa Monographs 2), Buffalo 1970.

Simelon, Propriété en Lucanie = P. Simelon, La propriété en Lucanie depuis les Gracques jusq'à l'avènement des Sévères. Étude épigraphique (Coll. Latomus 220), Bruxelles 1993.

SINN, Marmorurnen $=$ F. SINN, Stadtrömische Marmorurnen (Beiträge zur Erschließung hellenistischer und kaiserzeitlicher Skulptur und Architektur 8), Mainz 1987.

SIRAGO, L'Italia agraria $=$ V. A. SIRAGO, L'Italia agraria sotto Traiano (Recueil de travaux d'histoire et de philologie ser. 4, fasc. 16), Leuven 1958.

Sirago, Sannio romano $=$ V. A. Sirago, Il Sannio romano. Caratteri e persistenze di una civiltà negata (Citra \& ultra 2), Napoli 2000.

SIRIS = L. VIDMAN (Ed.), Sylloge inscriptionum religionis Isiacae et Sarapiacae (RGVV 28), Berolini 1969.

Socciarelli, Sul lago Fucino = T. Brogi, Sul lago Fucino e sue escrescenze: progetti per bonificarlo colla descrizione dell'Emissario di Tiberio Claudio: e sulla necessità di riaprirlo. Da un manoscritto della Biblioteca Angelica di Roma auctore Tommaso Brogi [vide ad nomen]. Introduzione, trascrizione e note a cura di ANTONIO M. SocciaRELLI. In appendice: Descrizione di un'antica grotta idrofora di Carmelo Mancini, Cerchio (AQ) 2012.

Società romana = A. GIARDINA (Ed.), Società romana e impero tardoantico I-IV, Roma - Bari 1986.

Société romaine $=\mathrm{R}$. BAUDRY $-\mathrm{S}$. DestePHEN (Ed.), La société romaine et ses élites. Hommages à Élisabeth Deniaux, Paris 2012.

SöDERSTRöm, Epigraphica Latina $=$ S. SöDERSTRÖM, Epigraphica Latina Africana. De titulis sepulcralibus prosa oratione compositis provinciarum Byzacenae et Proconsularis quaestiones selectae, Upsaliae 1924.

Sogliano, Museo Provinciale = A. Sogliano, Il Museo Provinciale Sannitico di Campobasso. Inventario degli oggetti antichi, Napoli 1889.

Solin, Analecta Epigraphica $=\mathrm{H}$. Solin, Analecta Epigraphica 1970-1997 iterum edenda indicibusque instruenda curavit M. KAJAva adiuvantibus K. KoRHOnen, M. Leiwo, O. Salomies (Acta Inst. Rom. Finl. 21), Roma 1998.

Solin, Beiträge zur Kenntnis = H. Solin, Beiträge zur Kenntnis der griechischen Personennamen in Rom I (Soc. Scient. Fennica, Comment. Human. Litt. 48), Helsinki 1971.

Solin, Epigraphische Untersuchungen $=$ H. SolIn, Epigraphische Untersuchungen in Rom und Umgebung (Annales Acad. Scient. Fennicae Ser. B, 192), Helsinki 1975.

Solin, Iscrizioni antiche $=\mathrm{H}$. SolIN, Le iscrizioni antiche di Trebula, Caiatia e Cubulteria, Caserta 1993.

Solin, Miscellanea atinate $=\mathrm{H}$. Solin, Miscellanea atinate, San Donato Val di Comino (FR) 2013. 
Solin, Namenbuch ${ }^{2}=$ H. Solin, Die griechischen Personen namen in Rom. Ein Namenbuch ${ }^{2}$ I-III (CIL Auctarium. Series nova 2), Berlin - New York 2003.

Solin, Namenpaare = H. Solin, Namenpaare. Eine Studie zur römischen Namengebung (Soc. Scient. Fennica, Comment. Human. Litt. 90), Helsinki 1990.

Solin, Sklavennamen $=$ H. Solin, Die stadtrömischen Sklavennamen. Ein Namenbuch I-III (Forsch. Sklaverei 2), Stuttgart 1996.

Solin - Salomies, Repertorium ${ }^{2}=$ H. Solin - O. SAlomies, Repertorium nominum gentilium et cognominum Latinorum. Editio nova addendis corrigendisque augmentata (Alpha-Omega, Reihe A 80), Hildesheim - Zürich - New York 1994.

SOMA $=$ O. Menozzi - M. L. Di Marzio - D. Fossataro (Ed.), SOMA 2005. Proceedings of the IX Symposium on Mediterranean Archaeology, Chieti (Italy), 24-26 February 2005 (BAR. International Series 1739), Oxford 2008.

SoRDINI, ms. I = G. SordinI, Nuovi frammenti epigrafici di Norcia (liber a. 1910 circ. manu exaratus apud Sezione di Archivio di Stato di Spoleto adservatus, Fondo Sordini 1, fasc. 3).

Sordini, ms. II = G. SordinI, Pugillaris (liber a. 1908 manu exaratus apud Sezione di Archivio di Stato di Spoleto adservatus, Fondo Sordini, $2^{\circ}$ acquisizione).

SoRDINI, $m s$. III = G. SORDINI, Schede epigrafiche del territorio di Cascia (liber a. 1892 circ. manu exaratus apud Sezione di Archivio di Stato di Spoleto adservatus, Fondo Sordini IV, fasc. 41).

Spadoni, Antiquaria = M. C. Spadoni Cerroni, Reate II. L'antiquaria (Bibl. Stud. Antichi 83), Pisa 1998.

Spadoni, I Sabini nell'antichità = M. C. Spadoni, I Sabini nell'antichità. Dalle origini alla romanizzazione, Rieti 2000.

Spadoni, Perugia $=$ M. C. Spadoni, Perugia romana $($ Dep. Umbria. Appendici al Bollettino 34), Perugia 2017.

Spadoni, Prefetti $=$ M. C. Spadoni, I prefetti nell'amministrazione municipale dell'Italia romana (Documenti e studi 39), Bari 2004.

Spadoni Cerroni, Rieti $=$ M. C. Spadoni Cerroni, Rieti in età romana, in M. C. Spadoni Cerroni - A. M. RegGiani Massarini, Reate [I.] (Bibl. Stud. Antichi 68), Pisa 1992, $9-124$.

SPEIDEL, Kaiserreiter $=$ M. P. SPEIDEL, Die Denkmäler der Kaiserreiter. Equites singulares Augusti (Beihefte der Bonner Jabrb. 50), Köln - Bonn 1994

Speidel, Roman Army Studies I-II = M. P. SPeIdel, Roman Army Studies I-II (Mavors 1. 8), Amsterdam 1984/92.

Splendore, Castel di Sangro = E. Splendore, Le antichità di Castel di Sangro. Guida illustrata alle epigrafi, Castel di Sangro (AQ) 1983.

SPlendore, Profilo archeologico = E. Splendore, Profilo archeologico e storico dei comuni della Valle Subequana (Contr. Valle Peligna. Quaderno 11), Castelvecchio Subequo (AQ) 1997.

SPLENDORE, Superaequum $=$ E. SPLENDORE, Superaequum e $i$ Peligni Superequani, Sulmona (AQ) 1979.

Spurii lapides = F. GALLO - A. SARTORI (Ed.), Spurii lapides. I falsi nell'epigrafia latina. Atti del Convegno, Milano, 2526 maggio 2016 (Ambrosiana Graecolatina, 8), Milano 26 magsi 2018.

SQuilla, Valle Roveto = G. SQuilla, Valle Roveto (L'Aquila) nella geografia e nella storia, Avezzano (AQ) - Casamari (FR) 1966.

ŠTAERMAN - Trofimova, La schiavitù = E. M. ŠTAERMAN M. K. Trofimova, La schiavitù nell'Italia imperiale, Roma 1975 (translatio Italica principis editionis Russicae 1971).
STALINSKI, Ritrovamento $=$ A. STALINSKI, Il ritrovamento di "Valle Fuino" presso Cascia. Analisi storico-culturale intorno ad un deposito votivo in alta Sabina (Mem. Pontif. Accad. Arch. s. 3 ${ }^{\circ}, 5$ ), Roma 2001.

Stanco, Mausoleo = E. A. Stanco, Il mausoleo degli 'Acilii Glabriones' ad Alife e i sepolcri a tamburo su podio con camera a cupola (I Quaderni di Oebalus 4), Roma 2013.

StARr, Navy ${ }^{2}=$ C. G. STARR, Roman Imperial Navy 31 B. C. - A. D. $324^{2}$, Cambridge 1960.

Statuti municipali $=$ L. Capogrossi Colognesi - E. GabBa (Ed.), Gli statuti municipali (Pubblicazioni del CEDANT 2), Pavia 2006.

A. Stein, Legaten von Moesien = A. Stein, Legaten von Moesien (Diss. Pann. ser. I. 11), Budapest 1940.

A. SteIn, Ritterstand $=$ A. STEIN, Der römische Ritterstand. Ein Beitrag zur Sozial- und Personengeschichte des römischen Reiches (MBPAR 10), München 1927 (lucis ope iterum impressum München 1963).

A. SteIn, Thracia $=$ A. STEIn, Römische Reichsbeamte der Provinz Thracia, Sarajevo 1920

E. Stein - Ritterling, Beamte = E. Stein - E. Ritterling, Die kaiserlichen Beamten und Truppenkörper im römischen Deutschland unter dem Prinzipat (Beiträge zur Verwaltungs- und Heeresgeschichte von Gallien und Germanien 1), Wien 1932 (lucis ope iterum impressum Amsterdam 1965)

STEK, Cult places $=$ T. D. STEK, Cult places and cultural change in republican Italy. A contextual approach to religious aspects of rural society after the Roman conquest (Amsterdam archaeological studies 14), Amsterdam 2009.

Stelluti, Epigrafi di Larino = N. Stelluti, Epigrafi di Larino e della Bassa Frentania I. Il repertorio, Campobasso 1997.

STERNINI, Romanizzazione $=$ M. STERNINI, La romanizzazione della Sabina Tiberina (Bibl. Arch. 13), Bari 2004.

Stevenson, $m s .=$ H. [= E.] Stevenson Junior, Libri saec. XIX p. m. manu scripti in Bibliotheca Apostolica Vaticana adservati sign. Vaticani Latini 10547-10587.

Storia dei Marsi I-III = MuZIo Febonio, Storia dei Marsi I-III, ed. G. Butticci - U. M. Palanza, Roma 1985/91.

Storia del Mezzogiorno I = G. Galasso - R. Romeo (Ed.), Storia del Mezzogiorno I. 2. Il Mezzogiorno antico, Napoli 1991.

Storia di Ortucchio = A. RADMILli - U. IRTI - G. Grossi - M. Mastroddi (Ed.), Storia di Ortucchio I. Dalle origini alla fine del Medioevo (Studi e testi 16), Roma 1985.

Storia di Pratola $=$ M. A. Petrella (Ed.), Viaggio nella storia di Pratola I. Dal Lago Peligno ai Celestini, Raiano (AQ) 2014.

Storia di un lago $=$ [M. РомıLIo (Ed.)], Il Fucino. Storia di un lago senz'aqua, Milano 1977.

Storia e archeologia $\mathrm{I}=\mathrm{G}$. Volpe - M. J. Strazzulla - D. LeONe (Ed.), Storia e archeologia della Daunia. In ricordo di Marina Mazzei. Atti delle giornate di studio, Foggia, 19 21 maggio 2005 (Insulae Diomedeae 8), Bari 2008.

Storia e archeologia II = G. Volpe (Ed.), Storia e archeologia globale dei paesaggi rurali in Italia fra Tardoantico $e$ Medioevo (Insulae Diomedeae 34), Bari 2018.

STORONi MAzzolani, Iscrizioni funerarie $=$ L. STORONI MaZZOLANI (Ed.), Iscrizioni funerarie, sortilegi e pronostici di Roma antica. Introduzione di G. CERONETTI, Torino 1973.

Strazzulla, Santuario sannitico ${ }^{2}=$ M. J. Strazzulla, Il santuario sannitico di Pietrabbondante ${ }^{2}$ (Documenti 1), [Campobasso] 1973.

Structures agraires = G. CHOUQUER - M. CLAVEL-LÉVÊQUE F. FAVORY - J.-P. VALlat (Ed.), Structures agraires en Italie centro-méridionale. Cadastres et paysage ruraux (Coll. de l'École Fr. de Rome 100), Paris - Roma 1987. 
Strutture agrarie $=$ E. GABBA - M. PASQUINUCCI, Strutture agrarie e allevamento transumante nell'Italia romana (IIII sec. a. C.) (Bibl. Stud. Antichi 18), Pisa 1979.

Strutture portuali $=$ C. ZACCARIA (Ed.), Strutture portuali e rotte marittime nell'Adriatico di età romana. Atti della XXIX Settimana di Studi Aquileiesi, 20-23 maggio 1998 (Ant. Alt. 46. Coll. de l'École Fr. de Rome 280), Trieste Roma 2001.

Studi Albino Garzetti = C. STella - A. Valvo (Ed.), Studi in onore di Albino Garzetti (Commentari dell'Ateneo di Brescia. Supplemento), Brescia 1996.

Studi Calderini - Paribeni $=$ Studi in onore di Aristide Calderin e Roberto Paribeni I-III, Milano 1956/57.

Studi Daniele Manacorda = E. Midolo et AL. (Ed.), Una lezione di archeologia globale. Studi in onore di Daniele Manacorda (Bibl. Arch. 52), Bari 2019.

Studi di urbanistica antica $=$ Studi di urbanistica antica (Quad. Ist. Top. Roma 2), Roma 1966.

Studi Francesco Grelle = M. Silvestrini - T. SPagnuolo Vigorita - G. Volpe (Ed.), Studi in onore di Francesco Grelle (Insulae Diomedeae 16), Bari 2006.

Studi Fulviomario Broilo = G. Cresci Marrone - A. PistedLATO (Ed.), Studi in ricordo di Fulviomario Broilo. Atti del Convegno, Venezia, 14-15 ottobre 2005 (Quaderni del Dipartimento di Scienze dell'Antichità e del Vicino Oriente, Università Ca’ Foscari Venezia 2), Padova 2007.

Studi Giorgio Gullini = M. Barra Bagnasco - M. C. ContI (Ed.), Studi di archeologia classica dedicati a Giorgio Gullini per i quarant'anni di insegnamento, Alessandria 1999.

Studi Giovanni Brusin = Studi aquileiesi offerti il 7 ottobre 1953 a Giovanni Brusin nel suo $70^{\circ}$ compleanno, Aquileia (UD) 1953

Studi Giuseppe Papponetti = L. Curreri - G. Traina (Ed.), Studi in onore di Giuseppe Papponetti (Le bandiere 12), Cuneo 2013.

Studi Jean Coste $=$ Z. Mari - M. T. Petrara - M. Sperandio (Ed.), Il Lazio tra antichità e medioevo. Studi in memoria di Jean Coste, Roma 1999.

Studi Luciano Agostiniani = G. M. Facchetti (Ed.), Mlax mlakas. Per Luciano Agostiniani (Quaderni di scienze del linguaggio 24), Milano 2008.

Studi Salvatore Calderone $=$ L. Di SALvo - M. Mazza - A Pinzone (Ed.), Hestíasis. Studi di tarda antichità offerti a Salvatore Calderone I-VI (Studi tardoantichi 1-6), Messina 1986/95

Studi Salvatore Riccobono = Studi in onore di Salvatore Riccobono nel XL anno del suo insegnamento I-IV, Palermo 1936.

Studi storico-epigrafici $=$ H. Solin (Ed.), Studi storico-epigrafici sul Lazio antico (Soc. Scient. Fennica, Comment. Human. Litt. 137), Helsinki 2019.

Studi su Praeneste = F. CoArelli (Ed.), Studi su Praeneste (Reprints di archeologia e di storia antica 1), Perugia 1978.

Studi sulle magistrature $=$ E. CAMPANILE - C. LeTta, Studi sulle magistrature indigene e municipali in area italica (Orientamenti linguistici 11), Pisa 1979.

Studi sull'Italia = R. CAPELLI (Ed.), Studi sull'Italia dei Sanniti. In occasione della Mostra "Italia dei Sanniti" (Roma, Museo Nazionale Romano, Terme di Diocleziano, 14 gennaio - 19 marzo 2000), Milano 2000.

Studi Vincenzo Orioles = R. Bомвi - F. Costantini (Ed.), Percorsi linguistici e interlinguistici. Studi in onore di Vincenzo Orioles (Tracce. Itinerari di ricerca), Udine 2018.

Studia Georgi Mihailov = A. Fol - D. BoJADZIEv (Ed.), Studia in honorem Georgii Mihailov, Sofia 1995.

Studia Géza Alföldy = W. EcK - B. FeHÉr - P. Kovács (Ed.), Studia epigraphica in memoriam Géza Alföldy (Antiquitas 1. 61), Bonn 2013.
Suburbium $=$ Ph. Pergola - R. Santangeli Veneziani - R. Volpe (Ed.), Suburbium. Il Suburbio di Roma dalla crisi del sistema delle ville a Gregorio Magno (Coll. de l'École Fr. de Rome 311), Rome 2003.

Sulmona = E. Mattiocco - G. Papponetti (Ed.), Sulmona, città d'arte e poeti, Pescara 1996

Suolahti, Junior Officers $=$ J. Suolahti, The Junior Officers of the Roman Army in the Republican Period. A Study on Social Structure (Annales Acad. Scient. Fennicae Ser. B, 97), Helsinki - Wiesbaden 1955.

Suriani, Monteodorisio = G. Suriani, Monteodorisio nel cuore, Vasto (CH) 1995.

SusI, Introdacqua = G. SusI, Introdacqua nella storia e nella tradizione, Sulmona (AQ) 1970.

Susini, Epigraphica dilapidata = G. SusinI, Epigraphica dilapidata. Scritti scelti (Epigrafia e Antichità 15), Bologna Faenza 1997.

Sviluppi recenti $=$ V. DE ANGelis (Ed.), Sviluppi recenti nella ricerca antichistica (Quaderni di Acme 54), Milano 2002.

Syme, Roman Papers I-VII = R. Syme, Roman Papers I-VII, Oxford 1979/91.

Syme, Ten Studies = R. Syme, Ten Studies in Tacitus, Oxford 1970.

SZRamkiewicz, Gouverneurs I-II = R. SZRamkiewicz, Les gouverneurs de province à l'époque augustéenne. Contribution à l'histoire administrative et sociale du principat III (Études prosopographiques 3-4), Paris 1975/76.

Tagliamonte, Figli di Marte = G. Tagliamonte, I figli $d i$ Marte. Mobilità, mercenari e mercenariato italici in Magna Grecia e Sicilia (Tyrrhenica. Studi archeologici sull'Italia antica 3), Roma 1994.

Tagliamonte, Sanniti = G. Tagliamonte, I Sanniti. Caudini, Irpini, Pentri, Carricini, Frentani (Biblioteca di archeologia 25), Milano 1996

TARPIn, Vici et pagi = M. TARPIN, Vici et pagi dans l'Occident romain (Coll. de l'École Fr. de Rome 299), Rome 2002.

Tarraco Biennal $=$ J. López VILAR (Ed.), Tarraco Biennal . Actes $1^{\text {er }}$ Congrès Internacional d'Arqueologia $i$ Món Antic. Govern i societat a la Hispània romana. Novetats epigràfiques. Homenatge a Géza Alföldy. Tarragona, 2930 de novembre $i 1$ de desembre de 2012, Tarragona 2013.

Tavola di Agnone = L. Del Tutto Parma (Ed.), La Tavola di Agnone nel contesto italico. Convegno di studio, Agnone, 13-15 aprile 1995 (Lingue e iscrizioni dell'Italia antica 7), Firenze 1996.

TAYLOR, Voting Districts = L. R. TAYLOR, The Voting Districts of the Roman Republic. The Thirty-five Urban and Rural Tribes (Pap. Mon. Am. Acad. Rome 20), Rome 1960 (ed. altera with updated material by JERZY LINDERSKI, Ann Arbor 2013).

Teate antiqua = V. Furlani (Ed.), Teate antiqua. La città di Chieti, Chieti 1991.

Temptanda viast $=$ C. Fernández Martínez - J. Gómez Pallarès (Ed.), Temptanda viast. Nuevos estudios sobra la poesía epigráfica latina, Bellaterra 2006.

Terme di Diocleziano I = R. Friggeri - M. G. Granino Cecere - G. L. Gregori (Ed.), Terme di Diocleziano. La collezione epigrafica, Milano 2012.

Terme di Diocleziano II = R. Friggeri - M. Magnani CiaNETTI - C. Caruso (Ed.), Terme di Diocleziano. Il chiostro piccolo della Certosa di Santa Maria degli Angeli, Milano 2014.

Terminavit sepulcrum = G. CResci Marrone - M. Tirelli (Ed.), Terminavit sepulcrum. I recinti funerari nelle necropoli di Altino. Atti del Convegno, Venezia, 3-4 dicembre 2003 (Studi e ricerche sulla Gallia Cisalpina 19. Altinum 4), Roma 2005

Terni - Interamna Nahars $=$ C. ANGelelli - L. Bonomi PonZI (Ed.), Terni - Interamna Nahars. Nascita e sviluppo di una 
città alla luce delle più recenti ricerche archeologiche (Coll. de l'École Fr. de Rome 362), Paris - Rome 2006.

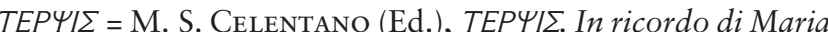
Laetitia Coletti (Università degli Studi "G. D’Annunzio" Chieti. Collana del Dipartimento di Scienze dell'Antichità. Sezione filologica 1), Alessandria 2002.

Terra dei Marsi = G. Luongo (Ed.), La Terra dei Marsi: cristianesimo, cultura, istituzioni. Atti del Convegno di Avezzano, 24-26 settembre 1998, Roma 2002.

Terremoti $=$ E. GuIdoboni (Ed.), I terremoti prima del Mille in Italia e nell'area mediterranea: storia, archeologia, sismologia, Bologna 1989.

Territorio = C. ЕвANISTA - M. RotILI (Ed.), Territorio, insediamenti e necropoli fra tarda antichità e alto medioevo. Atti del Convegno internazionale di studi "Territorio $e$ insediamenti fra tarda antichità e alto medioevo", Cimitile - Santa Maria Capua Vetere, 13-14 giugno 2013; Atti del Convegno internazionale di studi "Luoghi di culto, necropoli e prassi funeraria fra tarda antichità e medioevo", Cimitile - Santa Maria Capua Vetere, 19-20 giugno 2014, Napoli 2016

Territorio alifano $=$ L. Di Cosmo - A. M. Villucci (Ed.), Il territorio alifano. Archeologia, arte e storia. Atti del Convegno, S. Angelo d'Alife, 26 aprile 1987, S. Angelo d'Alife (CE) 1990.

Tesoro del lago = A. Campanelli (Ed.), Il tesoro del lago. L'archeologia del Fucino e la collezione Torlonia, Pescara 2001.

Tetraonyma = Tetraonyma . Miscellanea Graeco-romana [Lucae de Regibus, Paulino Mingazzini, Aldoni Neppi Modona, Henrico Turolla 70 aetatis annos felicissime explentibus Genuenses collegae discipulique] (Pubblicazioni dell'Istituto di Filologia Classica dell'Università di Genova 25), Genova 1966.

Theodor Mommsen = I. FARGNOLI - S. Rebenich (Ed.), Theodor Mommsen und die Bedeutung des römischen Rechts (Freiburger Rechtsgeschichtliche Abhandlungen 69), Berlin 2013.

Theodor Mommsen e il Lazio antico = F. MANNINO - M. MANNINO - D. F. Maras (Ed.), Theodor Mommsen e il Lazio antico. Giornata di Studi in memoria dell'illustre storico, epigrafista e giurista, Terracina, Sala Valadier, 3 aprile 2004 (Stud. Arch. 172), Roma 2009.

Thermes Romains $=$ Les thermes Romains. Actes de la table ronde organisée par l'École francaise de Rome, Rome, 1112 novembre 1988 (Coll. de l'École Fr. de Rome 142), Rome 1991.

Thielmann, Privatanktion = G. Thielmann, Die römische Privatanktion: zugleich ein Beitrag zum römischen Bankierrecht (Berliner juristische Abhandlungen 4), Berlin 1961.

Thomasson, Fasti = B. E. Thomasson, Fasti Africani. Senatorische und ritterliche Amsträger in den römischen Provinzen Nordafrikas von Augustus bis Diokletian (Acta Inst. Rom. Sueciae, Ser. in 4 ${ }^{\circ}$ 53), Stockholm 1996.

Thomasson, Laterculi I = B. E. Thomasson, Laterculi praesidum I. Lists of governors, Göteborg 1984.

Thomasson, Laterculi III = B. E. Thomasson, Laterculi praesidum III, Göteborg 1990

Thomasson, Legatus = B. E. Thomasson, Legatus. Beiträge zur römischen Verwaltungsgeschichte (Acta Inst. Rom. Sueciae, Ser. in $\left.8^{\circ}, 18\right)$, Stockholm 1991.

Thomasson, $S P Q R=$ B. Thomae $[=$ B. E. Thomasson $]$, $S P Q R$. Senatores procuratoresque Romani, Göteborg 1975.

Thomasson, Statthalter I-II = B. E. Thomasson, Die Statthalter der römischen Provinzen Nordafrikas von Augustus bis Diocletianus I-II (Acta Inst. Rom. Sueciae, Ser. in $8^{\circ}, 9$, 1-2), Lund 1960.
Thomsen, Italic Regions = R. Thomsen, The Italic Regions from Augustus to the Lombard Invasion (Classica et Medievalia. Dissertationes 4), København 1947 (lucis ope iterum impressum Roma 1966).

Thulin, Etruskische Disciplin = C. O. Thulin, Die etruskische Disciplin, Göteborg 1906/09 (lucis ope iterum impressum Darmstadt 1968).

Tirabasso, Bovianum Vetus = A. Tirabasso, Ubi Bovianum Vetus? Indagini sul sito di Bovianum osco-sannita prima della conquista romana, Campobasso 1929.

Tobalina OraÁ, Cursus honorum = E. Tobalina OraÁ, El cursus honorum senatorial durante la época Julio-Clandia (Mundo antiguo 10), Pamplona 2007.

Todisco, Veterani $=$ E. Todisco, I veterani in Italia in età imperiale (Documenti e studi 22), Bari 1999.

Todisco, Vici = E. Todisco, Vici rurali nel paesaggio dell'Italia romana (Documenti e studi 50), Bari 2011.

Tollis, Alba Fucense = C. Tollis, Alba Fucense dalle origini ai nostri giorni, Avezzano (AQ) 1961.

Tollis, Massa d'Albe = C. Tollis, Origini e vicende di Massa d'Albe (L'Aquila), Pescara 1977.

Tollis, Pacentro = C. Tollis, Pacentro. Storia, tradizione, leggenda, folklore dalla preistoria ad oggi, Sulmona (AQ) 1979.

TOMASSETTI, La campagna romana $\mathrm{III}=\mathrm{G}$. TOMASSETTI, $\mathrm{La}$ campagna romana antica, medievale e moderna III. Vie Cassia e Clodia, Flaminia e Tiberina, Labicana e Prenestina, ed. F. Tомаssetti, Roma 1913 (editio altera: G. TOMASSETTi, La campagna romana antica, medievale e moderna. Nuova edizione aggiornata a cura di L. Chiumenti $e$ F. Bilancia III. Vie Cassia e Clodia, Flaminia e Tiberina, Labicana e Prenestina, Firenze 1979).

ToppI, $m s .=$ N. ToppI, Adversaria quae recensent titulos nonnullos Histonii, Teate, Aterni vici et Pinnae (volumen sign. XXI. D. 27 saec. XVII medio exaratum a Nicolao ToppI, de quo nunc vide M. Spadaccini, OFIAB 90, 2010, 225 250, servatum Neapoli in Biblioteca della Società Napoletana di Storia Patria; esse videtur ac "volumen a Capasso postea Minieri Riccio datum hodie ubi extet nescio standumque fuit excerptis Capassi satis accuratis iis et plenis" ut ait Mommsen supra p. 317; gratias ago Umberto SolDOvIERI, qui me de adversariis Toppianis certiorem fecit, et Cristina Pepe, quae hoc volumen studiose contulit).

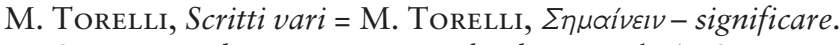
Scritti vari di ermeneutica archeologica, ed. A. SCIARMA, Pisa - Roma 2012.

M. R. Torelli, Benevento romana $=$ M. R. Torelli, Benevento romana (Saggi di storia antica 18), Roma 2002.

Tosi, Edifici di spettacoli = G. TosI, Gli edifici di spettacoli nell'Italia romana, Roma 2003.

Toynbee, Hannibal's Legacy = A. J. Toynbee, Hannibal's Legacy. The Hannibalic war's effect on Roman Life I-II, Oxford 1965.

Tra memoria e futuro = A. Di Fonzo (Ed.), Tra memoria e futuro. Sulmona e il suo territorio dall'archeologia ad Internet, Corfinio (AQ) 2001

Tran, Membres $=$ N. Tran, Les membres des associations romaines. Le rang social des collegiati en Italie et en Gaules sous le Haut-Empire (Coll. de l'École Fr. de Rome 367), Paris - Roma 2006.

Transhumance $=$ C. Jourdain-AnNeQuin - J.-C. Duclos (Ed.), Aux origines de la transhumance. Les Alpes et la vie pastorale d'hier à aujourd'bui, Paris 2006.

Transumanza = A. CAMPANELLI (Ed.), Giornate internazionali di studio sulla transumanza. Atti del Convegno, L'AquilaSulmona - Campobasso - Foggia, 4-7 novembre 1984, L'Aquila 1990.

Trasacco $=$ Trasacco. Guida turistica, Avezzano (AQ) 1996. 
Traverso, Esercito = M. Traverso, Esercito romano $e$ società italica in età imperiale I. I documenti epigrafici (Serta antiqua 10), Roma 2006.

Triantafillis, Iscrizioni italiche $=\mathrm{E}$. TRIANTAFILlis, Le iscrizioni italiche dal 1979. Testi, retrospettiva, prospettive (Quaderni patavini di linguistica. Monografie), Padova 2008.

Tribù romane $=$ M. SILvesTRINI (Ed.), Le tribù romane. Atti della XVI Rencontre sur l'épigraphie, Bari, 8-10 ottobre 2009 (Scavi e ricerche 19), Bari 2010.

Trionfo dell'acqua = Il trionfo dell'acqua. Acque e acquedotti a Roma IV sec. a.C. - XX sec. Mostra organizzata in occasione del $16^{\circ}$ Congresso ed Esposizione internazionale degli acquedotti. Roma, 31 ottobre 1986 - 15 gennaio 1987, Roma 1986.

Trivento $=$ Trivento. Cenni geografici, storici, archeologici. Documentazione fotografica, Trivento (CB) 1983 (libellus machina dactylographica scriptus).

Trutta, Dissertazioni = G. F. TRUTTA, Dissertazioni istoriche delle antichità alifane, Napoli 1776

Tuteri, Solymon $=$ Renato Tuteri, Solymon, Sulmo, Solmona, Sulmona I, Sulmona (AQ) 1980.

TUTERI, Dal silenzio = RosANnA TUTERI, ...dal silenzio: piccole storie dei cittadini di Sulmo nel museo archeologico, Sulmona 2002.

Ultimo Cesare = G. URso (Ed.), L'ultimo Cesare. Scritti, riforme, progetti, poteri, congiure. Atti del convegno internazionale, Cividale del Friuli, 16-18 settembre 1999 (CRDAC 20), Roma 2000.

Umbria $=$ F. ColivicCHI - C. ZACCAGNino (Ed.), Umbria . Con una presentazione di S. Rinaldi Tufi (Archeologia delle Regioni d'Italia), Roma 2008.

UnTERMANN, Wörterbuch $=$ J. UnTERMANN, Wörterbuch des Oskisch-Umbrischen (Handbuch der italischen Dialekte 3), Heidelberg 2000.

Uomini, istituzioni, mercati = M. MaIUro - G. D. Merola M. De NARdis - G. Soricelli (Ed.), Uomini, istituzioni, mercati. Studi di storia per Elio Lo Cascio (Pragmateiai 30), Bari 2019.

Usi e abusi = M. G. Angeli Bertinelli - A. Donati (Ed.), Usi e abusi epigrafici. Atti del Colloquio Internazionale di Epigrafia Latina, Genova, 20-22 settembre 2001 (Serta antiqua 6), Roma 2003.

Usus veneratioque fontium = L. GASPERINI (Ed.), Usus veneratioque fontium. Fruizione e culto delle acque salutari nell'Italia romana. Atti del Convegno Internazionale di Studio su "Fruizione e Culto delle Acque Salutari in Italia", Roma - Viterbo, 29-31 ottobre 1993, Tivoli (RM) 2006.

Utilitas necessaria $=$ G. BODON - I. RIERA - P. ZANOVELlO (Ed.), Utilitas necessaria. Sistemi idraulici nell'Italia romana, Milano 1994.

VAlente, Isernia $=\mathrm{F}$. VAlente, Isernia. Origine e crescita di una città. S. Diebner, Considerazioni sulle lapidi romane. E. TrotтA, Gli ultimi strumenti urbanistici, Campobasso E. Trot1 1982.

Valentetti, $m s .=$ A. ValentetTI, Storia di Cerreto di Spoleto (liber manu scriptus circ. a. 1890 servatus in bibliotheca archiepiscopali Spoletina sine inv. n.).

Valle del Fino = L. Franchi dell'Orto (Ed.), Dalla valle de Fino alla valle del medio e alto Pescara (Documenti Abruzzo Teramano VI. 1), Teramo 2003.

Valle del Piomba $=$ L. Franchi Dell'Orto (Ed.), Dalla valle del Piomba alla valle del basso Pescara (Documenti Abruzzo Teramano V. 1), Teramo 2001.

Valle del Salto = R. Pagano - C. Silvi (Ed.), Atti Giornata di Studi. Informazione e cultura per la protezione e valorizzazione del patrimonio storico ed archeologico della Valle del
Salto, S. Lucia di Fiamignano (Rieti), 1 dicembre 2007 (Quaderni Valle del Salto 1), Roma 2008.

Valle di Comino I = H. Solin (Ed.), Le epigrafi della Valle di Comino. Atti del Primo Convegno Epigrafico Cominese, Alvito, Palazzo Ducale, 5 giugno 2004, Casamari (FR) 2005.

Valle di Comino II = H. Solin (Ed.), Le epigrafi della Valle di Comino. Atti del Secondo Convegno Epigrafico Cominese, San Donato Val di Comino, 28 maggio 2005, Cassino (FR) 2006.

Valle di Comino III = H. Solin (Ed.), Le epigrafi della Valle di Comino. Atti del Terzo Convegno Epigrafico Cominese, San Donato Val di Comino, 27 maggio 2006, Cassino (FR) 2007.

Valle di Comino IV = H. Solin (Ed.), Le epigrafi della Valle di Comino. Atti del Quarto Convegno Epigrafico Cominese, Atina, Palazzo Ducale, 26 maggio 2007, Cassino (FR) 2008.

Valle di Comino V = H. Solin (Ed.), Le epigrafi della Valle di Comino. Atti del Quinto Convegno Epigrafico Cominese, Atina, Palazzo Ducale, 1 gingno 2008, Cassino (FR) 2009.

Valle di Comino VI = H. Solin (Ed.), Le epigrafi della Valle di Comino. Atti del Sesto Convegno Epigrafico Cominese, Atina, Palazzo Ducale, 31 maggio 2009, Cassino (FR) 2010.

Valle di Comino VII = H. Solin (Ed.), Le epigrafi della Valle di Comino. Atti del Settimo Convegno Epigrafico Cominese, Atina, Palazzo Ducale, 5-6 giugno 2010, San Donato Val di Comino (FR) 2011.

Valle di Comino VIII = H. Solin (Ed.), Le epigrafi della Valle di Comino. Atti dell'Ottavo Convegno Epigrafico Cominese, Atina, Palazzo Ducale, 28-29 maggio 2011, San Donato Val di Comino (FR) 2012.

Valle di Comino IX = H. Solin (Ed.), Le epigrafi della Valle di Comino. Atti del Nono Convegno Epigrafico Cominese, Alvito, Istituto Comprensivo "Mario Equicola", 13 ottobre 2012, San Donato Val di Comino (FR) 2013.

Valle di Comino X = H. Solin (Ed.), Le epigrafi della Valle di Comino. Atti del Decimo Convegno Epigrafico Cominese, Sora-Abbazia di S. Domenico, Sala "Riccardo Courrier", sabato 1 giugno 2013, San Donato Val di Comino (FR) 2014.

Valle di Comino XI = H. SolIn (Ed.), Le epigrafi della Valle di Comino. Atti dell'Undicesimo Convegno Epigrafico Cominese, Sora - Atina, 30-31 maggio 2014, Arezzo 2015.

Valle di Comino XII = H. Solin (Ed.), Le epigrafi della Valle di Comino. Atti del Dodicesimo Convegno Epigrafico Cominese, Atina, Palazzo Ducale, 29-30 maggio 2015, Arezzo 2016.

Valle di Comino XIII = H. Solin (Ed.), Le epigrafi della Valle di Comino. Atti del Tredicesimo Convegno Epigrafico Cominese, Atina, Palazzo Ducale, 28 maggio 2016, Arezzo 2017.

Valle di Comino XIV = H. Solin (Ed.), Le epigrafi della Valle di Comino. Atti del Quattordicesimo Convegno Epigrafico Cominese, Atina, Palazzo Ducale, 27-28 maggio 2017, Città di Castello (PG) 2018

Valle di Comino XV = H. Solin (Ed.), Le epigrafi della Valle di Comino. Atti del Quindicesimo Convegno Epigrafico Cominese, Atina, Palazzo Ducale, 2 giugno 2018, Arezzo 2019.

Valle Peligna $=$ M. Buonocore - O. Giannangeli - D. V. FuCINESE (Ed.), La Valle Peligna nella Romanità e nel Medioevo, Sulmona (AQ) 1991.

Valle Roveto $=$ La Valle Roveto e il Sacro: dal mondo antico al medioevo. Atti del $2^{\circ}$ Convegno di archeologia, Civita d'Antino, 14 settembre 2002, Roma 2006.

Valle Siciliana $=$ La Valle Siciliana o del Mavone (Documenti Abruzzo Teramano I. 1), Teramo 1983. 
Valnerina $=[$ B. Toscano (Ed.) $]$, La Valnerina, Spoleto $(\mathrm{PG})$ 1987.

van Der Ploeg, Asclepius = G. van der Ploeg, The Impact of the Roman Empire on the Cult of Asclepius (Impact of Empire 30), Leiden - Boston 2018.

Van Wonterghem, Antiche genti $=$ F. Van Wonterghem, Antiche genti peligne (Quad. Museo Sulmona 5), Sulmona (AQ) 1975.

Van Wonterghem, Corfinium = F. VAN Wonterghem, Cor finium van italische nederzetting tot romeins municipium, Diss. Leuven 1965.

Van Wonterghem, Forma Italiae $=$ F. VAn Wonterghem, Superaequum - Corfinium - Sulmo (Forma Italiae. Regio IV. Volumen I), Firenze 1984.

Van Wonterghem, Ritratti = F. Van Wonterghem, Ritratti della famiglia giulio-claudia nel territorio peligno (Documenti 2), Roma 1973.

Van Wonterghem, Superaequum $=$ F. VAn Wonterghem, Superaequum nel periodo romano (Contr. Valle Peligna. Quaderno 3), Castelvecchio Subequo (AQ) 1984.

VÁrhelyi, Religion = Zs. VÁrhelyi, The Religion of Senators in the Roman Empire. Power and the Beyond, Cambridge 2010.

Varia epigraphica $=$ M. G. Angeli Bertinelli - A. Donati (Ed.), Varia epigraphica. Atti del Colloquio Internazionale di Epigrafia, Bertinoro, 8-10 giugno 2000 (Epigrafia e Antichità 17), Bologna - Faenza 2001.

Venafrum = C. Ricci (Ed.), Venafrum città di Augusto. Tra coltura e cultura, topografia, archeologia e storia (Urbana species 3), Roma 2015.

Verani, Affreschi $=$ C. Verani, Gli affreschi votivi del battistero e della chiesa di Santa Maria extra moenia di Antrodoco, Rieti 2003.

Verga, Ager $=$ F. Verga, Ager Foronovanus I (Forma Italiae 44), Firenze 2006.

Vermaseren, Corpus Inscriptionum I-II = M. J. VermaseREN, Corpus Inscriptionum et Monumentorum Religionis Mithriacae I-II, Hagae Comitis 1956/60

VERUCCI, $m s .=$ L. VerUCCI, Memorie istoriche sagre e profane della città di Norcia (liber ante a. 1616 manu exaratus in codice I viri docti Rosa [vide supra] insertus f. 1-43).

Verzulli, Riofreddo = L. Verzulli, Le iscrizioni di Riofreddo (I Quaderni di Lumen 7), Pietrasecca (AQ) 2002.

Vestigia $=$ V. Gasparini (Ed.), Vestigia. Miscellanea di studi storico-religiosi in onore di Filippo Coarelli nel suo $80^{\circ}$ anniversario $(P A w B$ 55), Stuttgart 2016.

Vestini $=$ ST. Bourdin - V. D'ErCole (Ed.), I Vestini e il loro territorio dalla Preistoria al Medioevo (Coll. de l'École Fr. de Rome 494), Rome 2014.

VetTER, Handbuch = E. VeTter, Handbuch der italischen Dialekte I. Texte mit Erklärung, Glossen, Wörterverzeichnis (Handbuch der italischen Dialekte 1. Indog. Bibl. 25. 1), Heidelberg 1953.

Via Salaria I = E. Catani - G. Paci (Ed.), La Salaria in età antica. Atti del Convegno di studi, Ascoli Piceno - Offida - Rieti, 2-4 ottobre 1997 (Ichnia, serie seconda 1), Roma 2000.

Via Salaria II = E. CATANi - G. PaCI (Ed.), La Salaria in età tardoantica e altomedievale. Atti del Convegno di studi, Rieti - Cascia - Norcia - Ascoli Piceno, 28-30 settembre 2001 (Ichnia, serie seconda 3), Macerata - Roma 2007.

Via del sale = I. TozZI - C. VIRILI (Ed.), La via del sale, Via di Civiltà. Atti del Convegno di Antrodoco (Antrodoco, 14 maggio 2016), Santa Rufina di Cittaducale (RI) 2018.

Viajeros $=$ F. Marco Simón - F. Pina Polo - J. Remesal Rodríguez (Ed.), Viajeros, peregrinos y aventureros en el mundo antiguo (Colálecció Instrumenta 36), Barcelona 2010.
VICARI, Produzione $=$ F. VICARI, Produzione e commercio de $i$ tessuti nell'Occidente romano (BAR. International series 916), Oxford 2001.

A. Vico, Toffia Sabina = A. VIco, Brevi Memorie di Toffia Sabina (liber machina dactylographica d. $30 \mathrm{~m}$. Nov. a. 1958 redactus in bibliotheca abbatiae Farfensis sign. $S B$ MISC III, 3 servatus).

M. C. Vico, Cantalupo = M. C. VIco, Cantalupo in Sabina, Falconara (AN) 1986.

Vidman, Isis und Serapis = L. Vidman, Isis und Serapis bei den Griechen und Römern. Epigraphische Studien zur Verbreitung und zu den Trägern des ägyptischen Kultes (RGVV 29), Berlin 1970.

Vie della storia $=$ M. G. Angeli Bertinelli - A. Donati (Ed.), Le vie della storia. Migrazioni di popoli, viaggi di individui, circolazione di idee nel Mediteranneo antico. Atti del II Incontro Internazionale di Storia Antica, Genova, 6-8 ottobre 2004 (Serta antiqua 9), Roma 2006.

Vigliotti, Telesia $=$ N. Vigliotti, Telesia ... Telese. Due millenni, Marigliano (NA) 1985.

Villa S. Silvestro = F. CoArelli - F. Diosono (Ed.), I Templi ed il Forum di Villa S. Silvestro. La Sabina dalla conquista romana a Vespasiano. Catalogo della mostra (Cascia, Museo Civico di Palazzo Santi, 5 gingno - 30 novembre 2009), Roma 2009.

VILLE, Gladiature en Occident $=$ G. VILLE, La gladiature en Occident des origines à la mort de Domitien (Bibl. des Écoles Fr. d'Athènes et de Rome 245), Paris - Rome 1981.

VINCENT, Jouer pour la cité = A. VINCENT, Jouer pour la cité. Une histoire sociale et politique des musiciens professionels de l'Occident romain (Bibl. des Écoles Fr. d'Athènes et de Rome 371), Rome 2016.

Vincenti, Diana $=$ M. C. Vincenti, Diana. Storia, mito $e$ culto della grande dea di Aricia, Roma 2010.

VIsco, $m s$. = M. VISCO, Adversaria manu scripta servata Romae apud Archivio Centrale dello Stato. MPI. Dir. Gen. AABBAA. Antichità e Scavi. I versamento, $1^{\circ}$ serie, b. 15, fasc. 26. 14, cui titulus "1878-1880. Piedimonte d'Alife. Scavi" (gratias ago U. Soldovieri, qui me comiter certiorem fecit).

VIтI, Res publica Aeserninorum = A. VITI, Res publica Aeserninorum. Documenti epigrafici per una città del Sannio dal periodo augusteo alla decadenza. Correlazioni e riflessi in altri centri, Isernia 1982.

VITUcCI, Praefectura urbi = G. VITUcCI, Ricerche sulla praefectura urbi in età imperiale (sec. I-III), Roma 1956.

Voce concordi $=$ F. Mainardis (Ed.), Voce concordi. Scritti per Claudio Zaccaria (Ant. Alt. 85), Trieste 2016.

Vogel-Weidemann, Statthalter $=$ U. Vogel-Weidemann, Die Statthalter von Africa und Asia in den Jabren 14-68n. Chr. Eine Untersuchung zum Verhältnis Princeps und Senat (Antiquitas 1. 31), Bonn 1982.

Wachtel, Freigelassene $=\mathrm{K}$. Wachtel, Freigelassene und Sklaven in der staatlichen Finanzverwaltung der römischen Kaiserzeit von Augustus bis Diokletian, Berlin 1966.

$\mathrm{W}_{\mathrm{ACHTR}}$, Altlateinische Inschriften $=\mathrm{R}$. WACHTER, Altlateinische Inschriften. Sprachliche und epigraphische Untersuchungen zu den Dokumenten bis etwa 150 v. Chr. (Europäische Hochschulschriften XV. 38), Bern - Frankfurt - New York - Paris 1987.

Wagner, Dislokation $=$ W. WAgner, Die Dislokation der römischen Auxiliarformationen in den Provinzen Noricum, Pannonien, Moesien und Dakien von Augustus bis Gallienus (Neue deutsche Forschungen. Abt. Alte Geschichte 5), Berlin 1938.

WaltZing, Corporations I-IV = J. P. WALtZING, Étude historique sur les corporations professionnelles chez les romains depuis les origines jusqu'à la chute de l'Empire d'Occident I-IV, Louvain 1895/1900. 
Warmington, Remains $=$ E. H. WARMINGTON, Remains of Old Latin IV. Archaic Inscriptions (The Loeb Classical Library), London - Cambridge (MA) 1940 (lucis ope iterum impressum London - Cambridge (MA) 1967).

Weaver, Familia Caesaris = P. R. C. WeAver, Familia Caesaris. A Social Study of the Emperor's Freedmen and Slaves, Cambridge 1972.

Weinstock, Divus Iulius = S. Weinstock, Divus Iulius, Oxford 1971.

WeIss, Sklave = A. WeISs, Sklave der Stadt. Untersuchungen zur öffentlichen Sklaverei in den Städten des römischen Reiches (Historia Einzelschriften 173), Stuttgart 2004.

Wesch-Klein, Funus publicum = G. Wesch-Klein, Funus publicum. Eine Studie zur öffentlichen Beisetzung und Gewährung von Ehrengräbern in Rom und den Westprovinzen (HABES 14), Stuttgart 1993.

Wie die Blätter = D. KrömeR (Ed.), Wie die Blätter am Baum, so wechseln die Wörter. 100 Jahre Thesaurus linguae Latinae. Vorträge der Veranstaltungen am 29. und 30. Juni 1994 in München, Stuttgart - Leipzig 1995.

Wilmanns, Exempla = G. Wilmanns, Exempla inscriptionum Latinarum in usum praecipue academicum, Berolini 1873.

WinkLer, Noricum $=$ G. WInKLER, Die Reichsbeamten von Noricum und ihr Personal bis zum Ende der römischen Herrschaft (Österr. Akad. Wiss. Phil.-Hist. Klasse, Sitzungsberichte 261.2), Wien 1969.

Wiseman, New Men = T. P. Wiseman, New Men in the Roman Senate 139 B. C. - 14 A. D., Oxford 1971.

Wohlmayr, Kaisersaal $=$ W. WOHLmayr, Kaisersaal. Kultanlagen der Augustalen und munizipale Einrichtungen für das Herrscherhaus in Italien, Wien 2004.

Wojciechowski, Cultores Deorum $=$ P. Wojciechowski, Cultores Deorum. Stowarzyszenie religijne w Italii w okresie wczesnego cesarstwa I-III w.n.e., Toruń 2015

Women = E. HemelrijK - G. Woolf (Ed.), Women and the Roman City in the Latin West (Mnemosyne Supplements. History and Archaeology of Classical Antiquity 360), Leiden - Boston 2013.

WORDSWORTH, Fragments = J. WORDSWORTH, Fragments and Specimens of Early Latin with Introductions and Notes, Oxford 1874.

Work, Labour and Professions = K. Verboven - Chr. LAFs (Ed.), Work, Labour and Professions in the Roman World (Impact of Empire 23), Leiden - Boston 2016.
Wrede, Herme $=$ H. Wrede, Die antike Herme, Mainz 1986. Zandegiacomi, Arsoli $=$ G. Zandegiacomi, Le lapidi $d i$ Arsoli. 22 secoli di storia scritta sulla pietra, Subiaco (RM) 1993.

ZANDER, Versus $=$ C. ZANDER, Versus Italici antiqui, Lundae 1890.

Zarker, Studies = J. W. Zarker, Studies in the Carmina Latina Epigraphica, Diss. Princeton 1958.

ZAZZA, ms. = A. ZAZZA, liber post a. 1881 manu scriptus, cui titulus Notizie di Carsoli, quem publici iuris fecerunt $\mathrm{M}$. SCIÒ - F. AMici - G. Alesssandri, Pietrasecca (AQ) 1998.

Zecca, Manuale = V. ZeccA, Manuale di Storia Patria (liber manu scriptus saec. XIX post medium Chieti servatus in Biblioteca Provinciale “A. Camillo de Meis" sign. n. LXXXVII).

ZeccA, Note = V. ZeCCA, Note archeologiche, Chieti 1911

ZECCA, Nuove scoperte $=\mathrm{V}$. ZECCA, Nuove scoperte archeologiche in Chieti, Chieti 1901.

ZeCCA, Scavi I = V. ZeCCA, Gli scavi della via Ulpia in Chieti. Studio archeologico, Teramo 1897.

Zecca, Scavi II = V. ZecCA, Gli scavi della via Ulpia $\left(2^{\circ}\right.$ ed ultimo tratto) in Chieti. Studio archeologico, Chieti 1899/ 1900.

ZeCCA, Topografia = V. ZeCCA, Topografia e corografia marrucina studiate ne' monumenti, Chieti 1889.

ZeCCA, Turri Valignani = V. ZecCA, Turri Valignani. Ricerca delle sue antichità e note storiche artistiche sulla chiesa medioevale de' SS. Giovanni Evangelista e Vincenzo Martire, L'Aquila 1905.

Zelazowski, Honos bigae $=\mathrm{J}$. Żelazowski, Honos bigae. Le statue onorarie romane su biga (Światowit. Supplement series A. 7), Varsavia 2001.

Zenodocchio, Viabilità = S. Zenodocchio, Antica viabilità in Abruzzo, L'Aquila 2008.

ZIMMER, Berufsdarstellungen = G. ZIMMER, Römische Berufsdarstellungen (Archäologische Forschungen 12), Berlin 1982.

Zinanni, Rocca di Botte = D. ZinannI, Da Rocca di Botte a Trevi. Pietro Eremita, l'uomo della speranza, Roma 1988. ZveTAIEFF, Inscriptiones Italiae Inferioris = I. ZveTAIEFF Inscriptiones Italiae Inferioris dialecticae, Mosquae 1886.

ZvetaiefF, Inscriptiones Italiae Mediae = I. ZveTAIEFF Inscriptiones Italiae Mediae dialecticae, Lipsiae 1884/85.

\section{PERIODICA SERIESQVE ABBREVIATE LAVDATAE}

In hoc catalogo periodica et series et editiones titulorum non finitae reperiri possunt

$A A=$ Archäologischer Anzeiger. Beiblatt zum Jahrbuch des Deutschen Archäologischen Instituts

Abh.d. Akad.d. Wiss. Göttingen, Phil.-hist. Kl. = Abhandlungen der Gesellschaft [der Akademie] der Wissenschaften in Göttingen, Philologisch-historische Klasse

Abruzzo = Abruzzo. Rivista dell'Istituto di Studi Abruzzesi

$A C=L^{\prime}$ Antiquité classique

Acme = Acme. Annali della Facoltà di Filosofia e Lettere dell'Università statale di Milano

Act. Class. = Acta Classica. Journal of the Classical Association of South Africa

Acta Ant. Compl. = Acta Antiqua Complutensia

Acta Ant. Hung. = Acta Antiqua Academiae Scientiarum Hungaricae

Acta Arch. Lov. = Acta Archaeologica Lovaniensia

Acta Arch. Slov. = Acta Archaeologica . Ljubliana, Académie slovene
Acta Inst. Rom. Finl. $=$ Acta Instituti Romani Finlandiae

Acta Inst. Rom. Sueciae = Acta Instituti Romani Regni Sueciae Acta Musei Napocensis = Acta Musei Napocensis. Prehistory Ancient History - Archaeology

Acta Musei Porolissensis = Acta Musei Porolissensis. Anuarul Muzeului de Iștorie si Artă di Zalău

$A E=$ L'Année Épigraphique. Revue des publications épigraphiques relatives à l'antiquité romaine

Aegyptus = Aegyptus. Rivista italiana di egittologia e di papirologia

Aequa = Aequa. Rivista di studi e ricerche sul territorio degli Equi

AEspA = Archivo Español de Arqueología

Aevum $=$ Aevum. Rassegna di Scienze storiche linguistiche e filologiche

$A G E A=$ Agenzia per le Erogazioni in Agricoltura 
AIPhO = Annuaire de l'Institut de Philologie et d'Histoire Orientales et Slaves

AJA = American Journal of Archaeology

AJAH $=$ American Journal of Ancient History

$A J P h=$ American Journal of Philology

Album pittorico = Album pittorico letterario abruzzese

Alm. Molise = Almanacco del Molise

Althistorisch-Epigraphische Studien = Althistorisch-Epigraphische Studien

Altri itinerari $=$ Altri itinerari. Arte, letteratura, natura, storia, tradizione

Anal. Rom. Inst. Dan. = Analecta Romana Instituti Danici

Anc. Soc. = Ancient Society

Ann. "Claudio Faina" = Annali della Fondazione per il Museo "Claudio Faina"

Ann. della Fac. di Lettere e Filosofia (Cagliari) $=$ Annali della Facoltà di Lettere, Filosofia e Magistero dell'Università degli Studi di Cagliari

Ann. della Fac. di Lettere e Filosofia (Macerata) $=$ Annali della Facoltà di Lettere e Filosofia dell'Università degli Studi di Macerata

Ann. della Fac. di Lettere e Filosofia (Perugia) $=$ Annali della Facoltà di Lettere e Filosofia dell'Università degli Studi di Perugia

Ann. Geoph. = Annals of Geophysics

Ann. Inst. = Annali dell'Instituto di Corrispondenza Archeologica

Ann. Ist. It. Stud. Stor. = Annali dell'Istituto Italiano per gli Studi Storici

Ann. Ist. Orient. Napoli = Annali dell'Istituto universitario orientale di Napoli. Archeologia e Storia Antica

Ann. Ist. Orient. Napoli Filol. $=$ Annali dell'Istituto universitario orientale di Napoli. Sezione Filologica

Ann. Ist. Orient. Napoli Ling. = Annali dell'Istituto universitario orientale di Napoli. Sezione Linguistica

Ann. Pisa = Annali della Scuola Normale Superiore di Pisa

Ann. Semin. Bari = Annali del Seminario giuridico economico della Regia Università di Bari

Ann. Univ. Sofia. Fac. Lettres = Annuaire de l'Université de Sofia, Faculté des Lettres

Annales Acad. Scient. Fennicae = Annales Academiae Scientiarum Fennicae

Annales Acad. Scient. Fennicae, Diss. Human. Litt. = Annales Academiae Scientiarum Fennicae, Dissertationes Humanarum Litterarum

Annuario = Annuario. Associazione storica del Sannio Alifano ANRW = Aufstieg und Niedergang der römischen Welt. Geschichte und Kultur Roms im Spiegel der neueren Forschung

Ant. Afr. = Antiquités Africaines. Histoire et archéologie de l'Afrique $d u$ Nord

Ant. Alt. = Antichità Altoadriatiche

Ant. tard. $=$ Antiquité tardive

Antichthon = Antichthon. Journal of the Australian Society for Classical Studies

Antike Welt $=$ Antike Welt. Zeitschrift für Archäologie und Kunstgeschichte

Antiqua = Antiqua. Pubblicazioni dell'Archeoclub

Antiquitas = Antiquitas. Abhandlungen zur alten Geschichte

Anz. Österr. Akad. Wiss. = Anzeiger der Österreichischen Akademie der Wissenschaften

Aquil. nostra $=$ Aquileia nostra. Rivista dell'Associazione nazionale per Aquileia

$A \leftrightarrow R=$ Atene e Roma

Arch. . Class. $=$ Archeologia Classica

Arch. Ephem. $=$ Archaiologike Ephemeri

Arch. Epigr. Mitt. Österr. = Archäologisch-Epigraphische Mittheilungen aus Österreich-Ungarn

Arch. Ert. = Archaeologiai Ertesito
Arch. Esp. Arq. = Archivo Español de Arqueología Arch. Glott. Ital. $=$ Archivio Glottologico Italiano Arch. Lex. Gram. = Archiv für lateinische Lexicographie und Grammatik mit Einschluß des älteren Mittelalters

Arch. Med. = Archeologia Medievale

Arch. [Reale] Soc. Rom. Stor. Patria = Archivio della [Reale] Società Romana di Storia Patria

Arch. Relig. = Archiv für Religionsgeschichte

Arch. Sott. $=$ Archeologia Sotterranea

Arch. Stor. Eccl. Umbria = Archivio per la Storia Ecclesiastica dell'Umbria

Arch. Stor. March. $=$ Archivio Storico Marchigiano

Arch. Stor. Mol. = Archivio Storico Molisano

Arch. Stor. Prov. Nap. = Archivio Storico per le province napoletane

Arch. Stor. Prov. Parm. = Archivio Storico per le province parmensi

Arch. Stor. Sannio Alifano = Archivio Storico del Sannio Alifano

Arch. Ven. = Archivio Veneto. Deputazione di Storia Patria per le Venezie

Archaeology = Archaeology. A publication of the Archaeological Institute of America

Archeo $=$ Archeo. Attualità del Passato. Istituto Geografico de Agostini

Archeologia $=$ Archeologia. Rocznik Instytutu Archeologii $i$ Etnologii Polskiej Akademii Nauk

Archeomolise = Archeomolise. Storia, archeologia e antropologia del Molise

Arctos $=$ Arctos. Acta Philologica Fennica

Athenaeum = Athenaeum. Studi di Letteratura e Storia dell'Antichità

ATTA = Atlante tematico di topografia antica

Atti Accad. Pontan. = Atti Accademia Pontaniana

Atti Accad. Rov. Agiati = Atti dell'Accademia Roveretana degli Agiati

Atti CESDIR = Atti del Centro Studi e Documentazione sull'Italia Romana

Atti Conv. Magna Grecia = Atti del Convegno di Studi sulla Magna Grecia

Atti Conv. Stud. Macer. = Atti del Convegno di Studi Maceratesi

Atti Ist. Veneto, Scienze, Lett., Arti = Atti dell'Istituto Veneto di Scienze, Lettere ed Arti, Classe di Scienze morali, Lettere ed Arti

Atti Mem. Acc. Patavina = Atti e Memorie dell'Accademia Patavina di Scienze, Lettere ed Arti

Atti Mem. Arcadia = Atti e Memorie dell'Arcadia

Atti Mem. Bologna = Atti e Memorie della Deputazione di Storia Patria per le Provincie di Romagna. Bologna

Atti Mem. Marche = Atti e Memorie della Deputazione di Storia Patria per le Marche

Atti Mem. Modena = Atti e Memorie Deputazione di Storia Patria per le Antiche Provincie Modenesi

Atti Mem. Soc. Tiburtina = Atti e Memorie della Società Tiburtina di Storia e d'Arte

Atti Mem. Treviso = Atti e Memorie dell'Ateneo di Treviso Atti Napoli = Atti dell'Accademia di Scienze Morali e Politiche. Società Nazionale di Scienze, Lettere ed Arti in Napoli

Atti Reale Accad. Arch. Napoli = Atti della Reale Accademia di Archeologia, Lettere e Belle Arti di Napoli

Atti Soc. Stor. Sannio = Atti della Società Storica del Sannio

Atti Terra Lavoro = Atti della Commissione Conservatrice dei Monumenti ed Oggetti di Antichità e Belle Arti nella Provincia di Terra di Lavoro

Aufidus = Aufidus. Rivista di scienza e didattica della cultura classica

Augustinianum = Augustinianum. Periodicum semestre Instituti Patristici "Augustinianum" 
Aurea Parma $=$ Aurea Parma . Rivista di storia, letteratura ed arte

BABesch = 1, $1926-44,1969$ : Bulletin van de Vereeniging tot Bevordering der Kennis van de Antieke Beschaving te 'sGravenhage; 45, 1970 - 82, 2007: Bulletin Antieke Beschaving. Babesch. Annual Papers on Mediterranean Archaeology; 83, 2008 sq.: BABESCH. Annual Papers on Mediterranean Archaeology

$B A R=$ British Archaeological Reports

BAV = Biblioteca Apostolica Vatican

Bayer. Akad. d. Wiss., Phil.-hist. Kl., Abh., N. F. = Bayerische Akademie der Wissenschaften, Philologisch-historische Klasse. Abhandlungen, Neue Folge

Bayer. Vorg.-Bl. = Bayerische Vorgeschichtsblätter

$B C A R=$ Bullettino della Commissione Archeologica Comunale di Roma

$B C H=$ Bulletin de Correspondance Hellénique

$B D A S P=$ Bullettino della Deputazione Abruzzese di Storia Patria

BHAC = Bonner Historia-Augusta-Colloquium

Bibl. Arch. = Bibliotheca archaeologica

Bibl. de l'École Haut. Ét. Sc. Hist. = Bibliothèque de l'École des Hautes Études, Sciences Historiques et Philologiques

Bibl. de l'École Haut. Ét. Sc. Rel. = Bibliothèque de l'École des Hautes Études, Sciences Religieuses

Bibl. de l'Inst. Franç. Naples = Bibliothèque de l'Institut Français de Naples

Bibl. Dep. Umbria = Biblioteca della Deputazione di Storia Patria per l'Umbria

Bibl. des Écoles Fr. d'Athènes et de Rome = Bibliothèque des Écoles Françaises d'Athènes et de Rome

Bibl. Stud. Antichi = Biblioteca di Studi Antichi

Boll. Antinori = Bollettino della Società di Storia Patria Anton Ludovico Antinori negli Abruzzi

Boll. Arch. $=$ Bollettino di Archeologia

Boll . Arte = Bollettino d'Arte del Ministero della Pubblica Istruzione

Boll. Ass. Ital. Cart. $=$ Bollettino della Associazione Italiana di Cartografia

Boll. Dep. Umbria = Bollettino della Deputazione di Storia Patria per l'Umbria

Boll. Dioc. Norcia $=$ Bollettino Diocesano di Norcia

Boll. Filol. Class. $=$ Bollettino di Filologia Classica

Boll. Mon. Mus. Gall. Pontif. = Bollettino. Monumenti, Musei e Gallerie Pontificie

Boll. Mus. Imp. Rom. = Bollettino del Museo dell'Impero Romano

Boll. Reg. Dep. Umbria = Bollettino della Regia Deputazione di Storia Patria dell'Umbria

Boll. Stor. Crem. $=$ Bollettino storico cremonese

Boll. Stor. Foligno = Bollettino storico della Città di Foligno Boll. Stor. Sub. = Bollettino storico-bibliografico subalpino

Boll. Stud. Lat. = Bollettino di Studi Latini

Bonner Jahrb. = Bonner Jahrbücher des Rheinischen Landesmuseums in Bonn und des Vereins von Altertumsfreunden im Rheinlande

$B R G K=$ Bericht der Römisch-Germanischen Kommission des Deutschen Archäologischen Instituts Berlin

Brill Studies Epigraphy = Brill Studies in Greek and Roman Epigraphy

BTCGI = G. NeNCI - G. VALLET (Ed.), Bibliografia topografica della colonizzazione greca in Italia e nelle isole tirreniche

Bull. Acad. Belgique = Bulletin de la Classe des Lettres et des Sciences Morales et Politiques de l'Académie Royale de Belgique

Bull. Arch. Crist. $=$ Bullettino di Archeologia Cristiana

Bull. Arch. Nap. = Bullettino Archeologico Napoletano
Bull. Inst. = Bullettino dell'Instituto di Corrispondenza Archeologica. Roma

Bull. Inst. Class. Stud. = Bullettin of the Institute of Classical Studies

Bull. Ist. Belge Rome = Bulletin de l'Istitut Historique Belge de Rome

Bull. Museo Civiltà Romana = Bullettino del Museo della Civiltà Romana (Suppl. ad Bullettino della Commissione Archeologica Comunale di Roma)

Bull. Seism. Soc. Amer. = Bulletin of Seismological Society of America

Bull. Trav. Hist. = Bulletin Archéologique du Comité des Travaux Historiques et Scientifiques

Bursians Jahresbericht $=$ Bursians Jabresbericht. Jahresbericht über die Fortschritte der klassischen Altertumswissenschaft

Caeculus $=$ Caeculus. Papers in Mediterranean Archaeology and Greek \& Roman Studies

Cahiers du Centre G. Glotz = Cahiers du Centre G. Glotz. Revue d'Histoire Ancienne publiée par le Centre de Recherches G. Glotz, Sorbonne

Cataloghi $=$ Cataloghi dei musei locali e delle collezioni del Lazio

CEDANT = Centro di studi e ricerche sui Diritti Antichi

CeSDIR = Atti del Centro Studi e Documentazione sull'Italia Romana

Chiron = Chiron. Mitteilungen der Kommission für Alte Geschichte und Epigraphik des Deutschen Archäologischen Instituts

Chronique d'Egypte = Chronique d'Égypte. Association de la Fondation Égyptologique Reine Elisabeth. Egyptologisch Genootschap Konigin Elisabeth

CISAM = Centro italiano di studi sull'alto medioevo

Civ. Catt. $=$ La Civiltà Cattolica

Class. Ant. = Classical Antiquity

Class. Med. = Classica et Mediaevalia. Revue danoise de philologie et d'histoire

Class. Philol. $=$ Classical Philology

Class. Quart. $=$ Classical Quarterly

Class. Review $=$ Classical Review

Clio $=$ Clio. Rivista trimestrale di studi storici

Coll. de l'École Fr. de Rome = Collection de l'École Française de Rome

Coll. Latomus $=$ Collection Latomus

Coll. Sodalizio = Colloqui del Sodalizio tra studiosi dell'arte

Columb. Stud. Class. = Columbia Studies in the Classical Tradition

Conoscenze $=$ Conoscenze. . Rivista annuale della Soprintendenza Archeologica per $i$ Beni Ambientali Architettonici Artistici e Storici del Molise

Conoscenze n. s. = Conoscenze. Rivista semestrale della Direzione Regionale per Beni Culturali e Paesaggistici del Molise

Considerazioni $=$ Considerazioni di Storia ed Archeologia

Contr. Ist. Fil. . Class. = Contributi dell'Istituto di Filologia Classica. Pubblicazioni dell'Università Cattolica del Sacro Cuore

Contr. Valle Peligna $=$ Contributi alla cultura della Valle Peligna Superequana

CRAI = Comptes Rendus de l'Académie des Inscriptions et Belles-Lettres

CRDAC = Centro Ricerche e Documentazione sull'Antichità Classica. Monografie

Cronachetta mensuale = Cronachetta mensuale di scienze naturali e d'archeologia redatta dal Prof. MARIANO Armellini

Cronichetta mensuale $=$ Cronichetta mensuale delle più importanti moderne scoperte nelle scienze naturali e loro applicazioni alle arti ed industria già redatta dalla $b . m$. di PIETRO 
Armellini continuata dal Prof. Tito Armellini e notizie archeologiche raccolte dal suo figlio MARIANo ARMELLINI CSIR = Corpus Signorum Imperii Romani Cultura Escrita $=$ Cultura Escrita \& Sociedad

Daidalos = Daidalos. Studi e ricerche del Dipartimento di scienze del mondo antico. Viterbo

DASP = Deputazione Abruzzese di Storia Patria

Dep. Umbria = Deputazione di storia patria per l'Umbria

Dial. Arch. = Dialoghi di Archeologia. Rivista semestrale

Dial. Hist. Anc. = Dialogues d'Histoire Ancienne

Dict. Antiq. = Dictionnaire des antiquités grecques et romaines Diss. Pann. $=$ Dissertationes Pannonicae

Diss. Pontif. Accad. Arch. = Dissertazioni della Pontificia Accademia Romana di Archeologia

Diz. epigr. = Dizionario epigrafico di antichità romane

Documenti = Documenti di Antichità Italiche e Romane

Documenti Abruzzo Teramano = Documenti dell'Abruzzo Teramano

Documenti e studi = Documenti e studi. Collana del Dipartimento di Scienze dell'antichità dell'Università di Bari. Sezione storica

$E A A=$ Enciclopedia dell'arte antica, classica e orientale $E C=$ Enciclopedia Cattolica

Echo $=$ Echo. Université de Lausanne. Institut d'archéologie et d'histoire ancienne

$E E=$ Ephemeris Epigraphica

Egitto e Vicino Oriente = Egitto e Vicino Oriente. Riv. della sezione Egittologia e Scienze Storicche del Vicino Oriente dell'Università degli studi di Pisa

Electrum $=$ Electrum. Studia $z$ historii staro żytne

Emporium = Emporium . Rivista mensile illustrate d'arte e di cultura

$E O=$ Enciclopedia Oraziana

Eos $=$ Commentarii Societatis Philologae Polonorum

Ephem. Dac. $=$ Ephemeris Dacoromana

Epigr. Studien = Epigraphische Studien

Epigraphica $=$ Epigraphica . Periodico Internazionale di Epigrafia

$E P R O=$ Études Préliminaires aux Religions Orientales dans l'Empire romain

Erga - Logoi $=$ Erga - Logoi. Rivista di storia, letteratura, diritto e cultura dell'antichità

$E R I A C=$ Équipe de Recherche Interdisciplinaire sur les Aires Culturelles

Étud. . Class. = Les Études Classiques

Études = Études de philologie, d'archéologie et d'histoire anciennes

Eugesta $=$ Eugesta. Journal on Gender Studies in Antiquity

Euphrosyne = Euphrosyne. Revista de filologia clássica

Eutopia = Eutopia . Commentarii novi de antiquitatibus totius Europae

$E V=$ Enciclopedia Virgilian

Fast. Arch. = Fasti Archeologici. Annual Bulletin of Classical Archaeology

Forsch. Berichte $=$ Forschungen und Berichte

Forsch. Sklaverei $=$ Forschungen zur antiken Sklaverei

GASLA = Giornale abruzzese di scienze, lettere e arti

Gerión = Gerión. Revista de Historia Antigua

Giorn. arcadico = Giornale arcadico di scienze, lettere e art

Giorn. Ital. Filol. = Giornale Italiano di Filologia

Giorn. Scavi Pompei = Giornale degli Scavi di Pompei

Glotta = Glotta. Zeitschrift für griechische und lateinische Sprache

Gnomon = Gnomon. Kritische Zeitschrift für die gesamte klassische Altertumswissenschaft

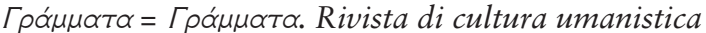

Gymnasium = Gymnasium. Zeitschrift für Kultur der Antike und humanistische Bildung

HABES = Heidelberger Althistorische Beiträge und Epigraphische Studien

Habis = Habis. Revista de la Universidad de Sevilla

Harvard Studies in Class. Philol. $=$ Harvard Studies in Classical Philology

Helikon = Helikon. Rivista di tradizione e cultura classica

Hephaistos = Hephaistos. Kritische Zeitschrift zur Theorie und Praxis der Archäologie, Kunstwissenschaft und angrenzender Gebiete

Hermes $=$ Hermes. Zeitschrift für klassische Philologie

Hesperìa $=$ Hesperìa . Studi sulla grecità di Occidente

Hisp. Ant. = Hispania Antiqua. Revista de Historia Antigua

Hisp. Epigr. = Hispania Epigraphica

Hist. Soc. Res. = Historical Social Research

Historia $=$ Historia. Zeitschrift für Alte Geschichte

Historiká = Historiká. Studi di storia greca e romana

"Opmos = "Opmos. Ricerche di storia antica

IASA = International Association of Sound and Audiovisual Archives

Ichnia = Ichnia . Collana del Dipartimento di Scienze Archeologiche e Storiche dell'Antichità dell'Università degli Studi di Macerata

$I C I=$ Inscriptiones Christianae Italiae septimo saeculo antiquiores

ICVR, N. S. = Inscriptiones Christianae urbis Romae septimo saeculo antiquiores, Nova Series [cf. supra inter opera breviate laudata $\mathrm{p}$. CLII].

$I G=$ Inscriptiones Graecae

Il bene comune = Il bene comune. Arte, cultura e civiltà per il terzo millennio

Illin. Studies = Illinois Studies in Language and Literature

Il Territorio $=$ Il Territorio. Rivista quadrimestrale di cultura e studi sabini

Incidenza dell'antico = Incidenza dell'antico. Dialoghi di storia greca

Incontri culturali $=$ Deputazione Abruzzese di Storia Patria Incontri culturali dei soci

Index = Index. Quaderni camerti di studi romanistici. International Survey of Roman Law

Indog. Bibl. = Indogermanische Bibliothek

Indog. Forsch. = Indogermanische Forschungen. Zeitschrift für Indogermanistik und allgemeine Sprachwissenschaft

Inscr. It. = Inscriptiones Italiae

Inst. Hist. Belge de Rome = Institute Historique Belge de Rome Insulae Diomedeae = Insulae Diomedeae. Collana di ricerche storiche e archeologiche

Inv. luc. = Invigilata lucernis

Itala Tellus = Itala Tellus. Studi di storia dell'Italia preromana e romana

Iura $=$ Iura. Rivista internazionale di diritto romano e antico

Jahreshefte d. Österr. Arch. Inst. = Jahreshefte des Österreichi schen Archäologischen Instituts.

JAT vide Riv. Top. Ant.

$\mathrm{JCPh}=$ Jahrbuch für classische Philologie

$J D A I=J a h r b u c h$ des Deutschen Archäologischen Instituts

$J E S=$ The Journal of Epigraphic Studies

$J F O=$ The Journal of Fasti Online

$J P h=$ Jabrbuch für Philologie

$J R A=$ Journal of Roman Archaeology

JRGZM = Jabrbuch des Römisch-Germanischen Zentralmuseums Mainz

$J R S=$ The Journal of Roman Studies

$J R T=$ Journal of Roman Topography

$J S=$ Journal des Savants 
Kleine Texte $=$ Kleine Texte für theologische und philologische Vorlesungen und Übungen

Klio $=$ Klio. Beiträge zur alten Geschichte

Koinonia = Koinonia . Rivista dell'Associazione di Studi Tardoantichi

Ktéma = Ktéma . Civilisations de l'Orient, de la Grèce et de Rome antiques

$L A D=$ Laboratorio di studi e ricerche sulle antiche province danubiane. Università degli Studi di Ferrara. Facoltà di Lettere e Filosofia. Dipartimento di scienze storiche

L'Africa Romana = L'Africa Romana. Atti dei Convegni di studio su l' "Africa romana"

L'Ant. Class. = L'Antiquité classique

Lanx = Lanx. Rivista elettronica della Scuola di Specializzazione in Archeologia dell'Università degli Studi di Milano

Lares $=$ Lares. Quadrimestrale di studi demoetnoantropologici

Latium = Latium. Rivista di studi storici

Latomus = Latomus. Revue d'études latines

Lavori e Studi = Lavori e Studi della Soprintendeza per i Beni Archeologici del Lazio

$L F=$ Listy Filologické

$L T L=$ Lexicon totius Latinitatis

Lumen = Il foglio di Lumen. Pubblicazione quadrimestrale dell'Associazione Culturale "Lumen"

Madr. Mitt. $=$ Madrider Mitteilungen

Maia = Maia . Rivista di letterature classiche

Mare internum $=$ Mare internum. Archeologia e culture del mediterraneo

Mat. Disc. Test. Class. = Materiali e discussioni per l'analisi dei testi classici

Maurolico = Il Maurolico. Giornale di storia, scienze, lettere e arti

Mavors = Mavors. Roman Army Researches

MBPAR = Münchener Beiträge zur Papyrusforschung und antiken Rechtsgeschichte

Meander = Meander. Miesiẹcznik poświẹcony kulturze świata starożytnego

Med. Antico = Mediterraneo Antico. Economie. Società. Culture. Rivista internazionale di Storia antica

MEFR = Mélanges d'Archéologie et d'Histoire de l'École Française de Rome

MEFRA = Mélanges de l'École Française de Rome. Antiquité MEFRM = Mélanges de l'École Française de Rome. Moyen$\hat{A} g e$

Mém. Acad. Inscr. Bell. Lett. $=$ Mémoires présentés par divers savants à l'Academie des Inscriptions et Belles-Lettres

Mem. Accad. Lincei = Atti della Accademia Nazionale dei Lincei. Classe di Scienze morali, storiche e filologiche. Memorie

Mem. Amer. Acad. Rome = Memoirs of the American Academy in Rome

Mem. Conn. Acad. Art. Scien.$=$ Memoires of the Connecticut Academy of Arts and Sciences

Mem. Ist. Veneto $=$ Memorie dell'Istituto Veneto

Mem. Pontif. Accad. Arch. = Memorie della Pontificia Accademia Romana di Archeologia

Mem. Reale Accad . Napoli = Memorie della Reale Accademia di Archeologia, Lettere e Belle Arti di Napoli

Mem. Soc. Istriana = Atti e Memorie della Società Istriana di Archeologia e Storia Patria

Mém. Soc. nat. Antiq. France $=$ Mémoires de la Societé nationale des antiquaires de France

Metis = Metis. Revue d'anthropologie du monde grec ancien

Metodi e prospettive $=$ Metodi e prospettive. Studi di Linguistica, Filologia, Letteratura

Min. Epigr. Papyr. = Minima Epigraphica et Papyrologica

Misc. Bibl. Ap. Vat. = Miscellanea Bibliothecae Apostolicae Vaticanae
Misc. Greca e Romana = Miscellanea Greca e Romana. Studi pubblicati dall'Istituto Italiano per la Storia Antica

Misc. Hadr. Med. = Miscellanea Hadriatica et Mediterranea

Mitt. Hist. Ver. Pfalz = Mitteilungen des Historischen Vereins der Pfalz

Mnemosyne = Mnemosyne. A journal of classical studies

Mon. Accad. Lincei = Monumenti antichi pubblicati per cura della Accademia Nazionale dei Lincei

Mon. Ant. Crist. $=$ Monumenti di Antichità Cristian

Monuments et Mémoires = Monuments et Mémoires publiés par l'Académie des Inscriptions et Belles-Lettres avec le concours de la Fondation Dourlans

Munera $=$ Munera . Collana di Studi storici sulla Tarda Antichità

Mus. Helv. = Museum Helveticum. Schweizerische Zeitschrift für klassische Altertumswissenschaft

Museo genti Abruzzo = Museo delle genti d'Abruzzo

Nouvelle Clio $=$ La Nouvelle Clio. Revue mensuelle de la découverte historique

Novitas = Novitas. Università Sulmonese della Libera Età

NS = Notizie degli Scavi di Antichità

Nuova Riv. Stor. = Nuova Rivista Storica

Nuovi Quad. Ist. Arch. Perugia = Nuovi Quaderni dell'Istituto di Archeologia dell'Università di Perugia

Nuovo Bull. Arch. Crist. $=$ Nuovo Bullettino di Archeologia Cristiana

Oebalus = Oebalus. Studi sulla Campania nell'Antichità

Österr. Akad. Wiss. Phil.-Hist. Klasse = Österreichische Akademie der Wissenschaften, Philosophisch-Historische Klasse

Oikumene = Oikumene. Studia ad historiam antiquam classicam et orientalem spectantem

Oltenia = Oltenia. Studii și comunicări. Arheologie și Istorie Veche

Opuscula Epigr. = Opuscula Epigraphica

Opuscula Inst. Rom. Finl. = Opuscula Instituti Romani Finlandiae

Opuscula Rom. = Opuscula Romana

Orizzonti $=$ Orizzonti. Rassegna di archeologia

Ostraka = Ostraka . Rivista di antichità

Palamedes $=$ Palamedes. A Journal of ancient history

Panorami = Panorami. Riflessioni, discussioni e proposte sul diritto e l'amministrazione

Pap. Mon. Am. Acad. Rome = Papers and Monographs of the American Academy in Rome

$P A w B=$ Potsdamer altertumswissenschaftliche Beiträge

PBSR = Papers of the British School at Rome

PCIA = Popoli e Civiltà dell'Italia Antica

Pegasus = Pegasus. Berliner Beiträge zum Nachleben der Antike

Philol. Monogr. Amer. = Philological Monographs of the American Philological Association

Philippika = Philippika. Marburger altertumskundliche Abhandlungen

Philologus = Philologus. Zeitschrift für antike Literatur und ibre Rezeption

Phoenix = Phoenix. The Journal of the Classical Association of Canada

Picus = Picus. Studi e ricerche sulle Marche nell'antichità

Planus = Planus. Quaderni di Studio dell'Istituto Teologico abruzzese-molisano

Polymnia = Polymnia . Studi di storia romana

$P P=L a$ parola del passato. Rivista di studi antichi

Pragmateiai = Pragmateiai. . Collana di studi e testi per la storia economica, sociale e amministrativa del mondo antico

Publications Louvain = Publications d'histoire de l'art $e$ d'archéologie de l'Université Catholique de Louvain

Puteoli $=$ Puteoli Studi di storia antica 
QFIAB = Quellen und Forschungen aus italienischen Archiven und Bibliotheken

QuadAEI = Quaderni del Centro di Studio per l'Archeologia Etrusco-Italica

Quad. Aquilano = Ente Provinciale per il Turismo - L'Aquila . Quaderni storico-artistici dell'Aquilano

Quad. Arch. Abruzzo = Quaderni di Archeologia d'Abruzzo. Notiziario della Soprintendenza per i Beni Archeologici dell'Abruzzo

Quad. Arte = I Quaderni dell'Arte

Quad. Coldragonesi $=$ Quaderni Coldragones

Quad. Conoscenza = I Quaderni della Conoscenza

Quad. Friul. Arch. = Quaderni Friulani di Archeologia

Quad. Ist. Arch. Chieti = Quaderni dell'Istituto di Archeologia e Storia antica dell'Università di Chieti

Quad. Ist. Glott. Bologna = Quaderni dell'Istituto di Glottologia dell'Università degli Studi di Bologna

Quad. Ist. Top. Roma = Quaderni dell'Istituto di Topografia Antica dell'Università di Roma

Quad. Ling. Urbino = Quaderni dell'Istituto di Linguistica dell'Università di Urbino

Quad. Museo Sulmona = Quaderni del Museo Civico di Sulmona

Quad. Storia $=$ Quaderni di Storia

Quad. Storia Ant. Epigr. = Quaderni di Storia Antica e di Epigrafia

Quad. Ticin. = Quaderni Ticinesi di numismatica e antichitò classiche

Quad. Urb. = Quaderni Urbinati di cultura classica

RAAM = Rassegna d'arte degli Abruzzi e del Molise

RAC = Reallexicon für Antike und Christentum. Sachwörterbuch zur Auseinandersetzung des Christentums mit der Antiken Welt

RASA = Rassegna abruzzese di storia e arte

RASL $=$ La Rivista abruzzese di scienze e lettere

RASLA = La Rivista abruzzese di scienze, lettere e arti

Rationes rerum $=$ Rationes rerum. Rivista di filologia e storia

RCCM = Rivista di cultura classica e medievale

$R E=$ Paulys Realencyclopädie der classischen Altertumswissenschaft

REA = Revue des Études Anciennes

REG $=$ Revue des Études Grecques

$R E L=$ Revue des Études Latines

$R G V V=$ Religionsgeschichtliche Versuche und Vorarbeiten

Rend. Accad. Arch. Napoli = Rendiconti della Accademia di Archeologia, Lettere e Belle Arti di Napoli

Rend. Accad. . Lincei = Atti della Accademia Nazionale dei Lincei. Classe di Scienze morali, storiche e filologiche. Rendiconti

Rend. Ist. Lomb. $=$ Rendiconti dell'Istituto Lombardo. Classe di Lettere e Scienze Morali e Storiche

Rend. Pontif. Accad. Arch. = Rendiconti della Pontificia Accademia Romana di Archeologia

RER $=$ Revue des Etudes Roumenes

Rev. Arch. $=$ Revue Archéologique

Rev. Arch. Narb. = Revue Archéologique de Narbonnaise

Rev. Belge de Philol. et d'Hist. = Revue Belge de Philologie et d'Histoire

Rev. Hist. de Droit Fr. et Étr. = Revue Historique de Droit Française et Étranger

Rev. Intern. de Droits de l'Ant. = Revue Internationale de Droits de l'Antiquité

Rev. Num. = Revue Numismatique

Rev. Philol. $=$ Revue de Philologie, de Littérature et d'Histoire Anciennes

Revista Bistriei $=$ Revista Bistriei. Un anuar de Complexul Muzeal Bistriţa Năsăud

Rhein. Mus. = Rheinisches Museum für Philologie
Rhesis $=$ Rhesis. International Journal of Linguistics, Philology and Literature

RIGI = Rivista Indo-Greco-Italica di filologia, lingua, antichità

RINASA = Rivista dell'Istituto Nazionale d'Archeologia e Storia dell'Arte

Riv. abruzzese = Rivista abruzzese. Rassegna trimestrale di cultura

Riv. Arch. = Rivista di Archeologia

Riv. Arch. Como = Rivista archeologica dell'antica provincia e diocesi di Como

Riv. Arch. Crist.$=$ Rivista di Archeologia Cristiana

Riv. Filol. = Rivista di Filologia e Istruzione Classica

Riv. Stor. Ant. = Rivista di Storia Antica

Riv. Stor. Antichità = Rivista Storica dell'Antichità

Riv. Stor. Benedettina = Rivista Storica Benedettina

Riv. Stor. Ital. = Rivista Storica Italiana

Riv. Stud. Liguri = Rivista di Studi Liguri

Riv. Stud. Pomp. = Rivista di Studi Pompeiani

Riv. Top. Ant. = Rivista di Topografia Antica (Journal of Ancient Topography

$R M=$ Mitteilungen des Deutschen Archäologischen Instituts. Römische Abteilung

Roc. Hum. = Roczniki Humanistyczne

Salternum $=$ Salternum. Semestrale di informazione storica, culturale e archeologica a cura del Gruppo Archeologico Salernitano

Samnium = Samnium. Rivista storica

Sandalion = Sandalion. Quaderni di cultura classica, cristiana e medievale

Sc. Ant. = Scienze dell'antichità. Rivista del Dipartimento di Scienze Storiche Archeologiche e Antropologiche dell'Antichità «Sapienza» Università degli Studi di Roma

Scripta antiqua $=$ Scripta antiqua . Collection des éditions Ausonius

Scriptorium = Scriptorium. Revue internationale des Études relatives aux manuscrits

SEBarc = Sylloge Epigraphica Barcinonensis

SEG = Supplementum Epigraphicum Graecum

Seia $=$ Seia . Quaderni di studi storico-archeologici dell'Antichità

Serta antiqua $=$ Serta antiqua e mediaevalia. Pubblicazione dell'Università degli Studi di Genova, Dipartimento di Scienze dell'Antichità e del Medioevo

Sic. Gymn. = Siculorum Gymnasium

Signifer = Signifer. Monografías y Estudios de Antigüedad Griega e Romana

$S L=$ Storia e Letteratura

Slavery and Abolition = Slavery and Abolition. A Journal of Slave and Post-Slave Studies

Soc. Scient. Fennica $=$ Societas Scientiarum Fennica

Soc. Scient. Fennica, Comment. Human. Litt. = Societas Scientiarum Fennica, Commentationes Humanarum Litterarum

Spoletium $=$ Spoletium. Rivista di arte storia cultura

Stud. Ant $=$ Studi di Antichita

Stud. Arch. = Studia Archaeologica

Stud. Benev. $=$ Studi Beneventani

Stud. . Class. Orient.$=$ Studi Classici e Orientali

Stud. Doc. Hist. Iuris = Studia et Documenta Historiae et Iuris Stud. Etr. = Studi Etruschi

Stud. Ist. It. Stor. Ant. = Studi pubblicati dal [Reale] Istituto per la Storia Antica / Studi pubblicati dall'Istituto Italiano per la Storia Antica

Stud. It. Filol. Class. = Studi Italiani di Filologia Classica

Stud. . Macer. = Studi Macerates

Stud. Magr. = Studi Magrebini

Stud. Merid. $=$ Studi Meridional

Stud. . Orient. Crist. $=$ Studi sull'Oriente Cristano

Stud. Phil. Valentina $=$ Studia philologica Valentina 
Stud. Picen.. Studia Picena

Stud. Ric. Matera = Siris. Studi e ricerche della Scuola di specializzazione in archeologia di Matera

Stud. Rom. = Studi Romani

Stud. Romagn. = Studi Romagnol

Stud. Saggi Ling. = Studi e Saggi Linguistici

Studi Ant. Crist.$=$ Studi di antichità cristiana pubblicati a cura del Pontificio Istituto di Archeologia Cristiana

Studi Miscellanei $=$ Studi Miscellanei. Seminario di Archeologia e Storia dell' Arte Grega e Romana dell'Università di Roma

Suppl. Comm. Ateneo di Brescia $=$ Supplemento ai Commentari dell' Ateneo di Bresci

Suppl. It. $=$ Supplementa Italica. Nuova serie .

Talanta $=$ Talanta . Atti della società archeologica e storica olandese

TAPhA $=$ Transactions of the American Philological Association

Taras $=$ Taras. Rivista di archeologia

Temporis signa $=$ Temporis signa. Archeologia della tarda antichità e del medioevo

Testi e manuali $=$ Testi e manuali per l'insegnamento universitario del Latino

ThCRA = Thesaurus Cultus et Rituum Antiquorum

ThLL $=$ Thesaurus linguae Latinae

Tituli = Tituli. Pubblicazioni di epigrafia e antichità greche e romane dell'Università di Roma - "La Sapienza" (postea "Sapienza" - Università di Roma)
Topoi $=$ Topoi Orient-Occident

Tyche $=$ Tyche. Beiträge zur alten Geschichte, Papyrologie und Epigraphik

Urbana species = Urbana species. Vita e città nell'Italia e nell'Impero romano

Veleia $=$ Veleia . Revista de prehistoria, historia antigua, arqueología y filología clásicas

Vestigia $=$ Vestigia. Beiträge zur alten Geschichte

Vesuviana = Vesuviana. An International Journal of Archaeological and Historical Studies on Pompeii and Herculaneum

Vet. Christ. $=$ Vetera Christianorum

Vetera $=$ Vetera . Ricerche di storia, epigrafia e antichità

Vichiana = Vichiana. Rassegna internazionale di Studi filologici e storici

WS = Wiener Studien. Zeitschrift für klassische Philologie und Patristik

Xenia $=$ Xenia. Semestrale di Antichità

$\mathrm{ZCP}=$ Zeitschrift für celtische Philologie

Zeitschr. d. Aach. Gesch. = Zeitschrift des Aachener Geschichts-

Zeitschr. d. Savigny-Stiftung, Rom. Abt. = Zeitschrift der SavignyStiftung für Rechtsgeschichte. Romanistische Abteilung

Zetemata $=$ Zetemata. Monographien zur Klassischen Altertumswissenschaft

$Z P E=$ Zeitschrift für Papyrologie und Epigraphik 
